\title{
Investigation of Sip1 gene interactions in the development of the mammalian telencephalon
}

\author{
PhD Thesis \\ in partial fulfilment of the requirements \\ for the degree "Doctor of Philosophy (PhD)/Dr. rer. nat." \\ in the Neuroscience Program \\ at the Georg August University Göttingen, \\ Faculty of Biology
}

submitted by

Anjana Nityanandam

born in

Chennai, India

Goettingen, 2009 


\section{Declaration}

This thesis has been written independently and with no other sources and aids than quoted.

Anjana Nityanandam

16 March 2009, Goettingen. 


\section{CONTENTS}

$\begin{array}{lr}\text { ABBREVIATIONS } & 1\end{array}$

$\begin{array}{lr}\text { ABSTRACT } & 5\end{array}$

1. INTRODUCTION

1.1 The developing neocortex $\quad 6$

$\begin{array}{ll}1.2 \text { Cortical progenitors } & \mathbf{8}\end{array}$

1.2.1 Neuroepithelial cells/Radial glia $\quad \mathbf{8}$

1.2.2 Basal Progenitors $\quad 9$

$\begin{array}{ll}\text { 1.3 Cell cycle in Corticogenesis } & \mathbf{1 0}\end{array}$

1.3.1 Regulation of neuron number $\quad \mathbf{1 0}$

1.4 Sequential generation of neurons and astrocytes in the cortex $\mathbf{1 1}$

1.5 Molecular control of Neurogenesis in the cortex $\quad \mathbf{1 2}$

1.6 Molecular control of Astrogliogenesis in the cortex 13

$\begin{array}{ll}\text { 1.7 Neuronal migration } & 15\end{array}$

1.7.1 Radial migration $\quad \mathbf{1 5}$

1.7.2 Tangential migration $\quad \mathbf{1 6}$

$\begin{array}{ll}1.8 \text { Smad- interacting protein } 1(\operatorname{Sip} 1) & \mathbf{1 6}\end{array}$

2. MATERIALS AND METHODS

2.1 Mouse mutants $\quad 19$

2.1.1 Conditional Sip1 knockout 19

2.1.2 Sip1-Sfrp1 double mutants $\quad \mathbf{1 9}$

2.1.3 Sip1-Fgfr1 double mutants $\quad \mathbf{2 0}$

2.1.4 Genotyping $\quad \mathbf{2 0}$

2.2 Tissue processing $\quad \mathbf{2 2}$

2.2.1 Perfusion $\quad \mathbf{2 3}$

2.3 Nissl Staining $\quad \mathbf{2 3}$

2.4 Immunohistochemistry $\quad \mathbf{2 4}$

2.4.1 Standard protocol (without amplification of signal) $\mathbf{2 4}$

2.4.2 Tyramide- based amplification of signal $\mathbf{2 4}$ 
2.6 Immunocytochemistry $\quad \mathbf{2 6}$

$\begin{array}{ll}2.7 \text { Image Acquisition and analysis } & \mathbf{2 7}\end{array}$

$\begin{array}{ll}2.8 \text { Western blotting } & \mathbf{2 7}\end{array}$

2.9 RNA Isolation, Microarray analysis and cDNA synthesis $\quad \mathbf{2 8}$

2.10 Semi- quantitative PCR (SQ-PCR) 29

2.11 In situ Hybridisation $\quad 30$

2.11.1 Transformation $\quad \mathbf{3 0}$

2.11.2 Plasmid isolation $\quad 30$

2.11.3 Plasmid linearization $\quad \mathbf{3 1}$

2.11.4 Purification of linearised plasmid DNA $\quad 31$

2.11.5 Riboprobe synthesis (for radioactive in situ hybridization) $\quad \mathbf{3 1}$

2.11.6 Radioactive in situ hybridization $\mathbf{3 2}$

2.12 Slice Culture $\quad \mathbf{3 3}$

2.12.1 Slice culture with Fgf9/NT3 treatment $\quad 33$

2.12.2 Slice culture with implantation of Fgf9/NT3 coated beads $\quad \mathbf{3 4}$

2.13 Slice overlay assay $\quad 35$

2.14 Explant culture (with NT3 treatment) 36

2.15 Dissociated cortical cell culture $\quad \mathbf{3 6}$

$\begin{array}{lr}\text { 3. RESULTS } & 37\end{array}$

3.1 Sip1 is expressed in postmitotic cells of the neocortex $\quad 37$

3.2 Conditional ablation of Sip1 in the cerebral cortex $\quad 39$

3.3 Morphology of Sip1- deficient cortices $\quad \mathbf{4 1}$

3.3.1 Thinner cortex with disorganised stratification of neurons,

absence of corpus callosum and anterior commissure $\quad \mathbf{4 1}$

3.3.2 Reduction in deep layer neurons accompanied by an expansion of upper layer cell types

3.3.3 Ablation of Sip1 has no effect on early proliferation and cell cycle kinetics of of cortical progenitors

3.3.4 Apoptosis is not enhanced during embryonic development 
3.3.5 Proliferation and specification of astrocytic precursors

during later stages of corticogenesis

3.3.6 Premature production of layer 2-5 neurons

3.3.7 Enhanced postnatal astrocytogenesis and premature

specification of glial progenitors in the neocortex

3.3.8 Increased proliferation continues to early postnatal stages in the absence of Sip 1

3.3.9 Hampered migration of neurons born at and after E15.5

3.4 Sip1- mediated signaling in the neocortex

3.4.1 Molecular targets of Sip1 in the cortex

3.4.2 Fgf signaling is affected in Sip1 mutant cortices

3.4.3 Exogenous application of Fgf9 to wildtype cortical

slices induces proliferation of Olig2 + glial precursors

3.4.4 Exogenous application of Neurotrophin-3 to wildtype cortical cells/explants is not sufficient to change the proportion of UL vs DL neurons

3.4.5 BMP signaling in ectopically dividing cells at E17.5

3.4.6 Notch signaling is not affected in Sip1 depleted cortices

3.4.7 Behaviour of early cortical progenitors on older

Sip1 deficient microenvironment

3.4.7.1 Differentiation of younger progenitors on older cortical slices

3.4.7.2 Differentiation of younger progenitors on older wildtype

versus mutant cortical slices

3.4.8 Deletion of Sfrp1 in Sip1 conditional knockouts does not rescue the neocortical defects seen in these mutants

\section{DISCUSSION}

4.1 Sip1 controls sequential cell fate switch in cortical progenitors during the course of embryonic development

4.2 Selective role of Sip1 in progenitor self- renewal in the cortex

4.3 Role of Sip1 in establishing the morphology of the neocortex 
4.4.1 Cortical neurons secrete Fgf9 that induces proliferation

of astrocytic precursors and/or a gliogenic fate switch of cortical progenitors

4.4.2 Premature expression of Neurotrophin-3 in Sip1- deficient

cortical plate is insufficient to induce DL to UL neuronal fate switch in progenitors

4.4.3 Differentiation of early cortical progenitors on older

wildtype and mutant cortices

4.5 Regulatory signaling pathways influenced by Sip1

4.5.1 Gliogenic feedback signaling induces BMP activity

in abventricular astrocytic precursors at E17.5

4.6 Molecular targets of Sip1 in the neocortex

4.7 Mixed identity and/or delayed maturation of some layer 5/6 cortical neurons in Sip1 conditional mutants

4.8 Upregulation of Wnt antagonist Sfrp1 is not responsible for enhanced gliogenesis in Sip1 conditional mutants 


\section{ABBREVIATIONS}

$\mathrm{AC}$

AOBS

APS

BG

bHLH

Blbp

BMP

bp

BrdU

BSA

$\mathrm{CC}$

cDNA

CGE

cHBSS

CMV

CNS

CNTF

$\mathrm{CP}$

cpm

CR

CT-1

DAPI

DL

DMEM

DMSO

DNA

dNTP

DTT

E

EDTA
Anterior Commissure

Acousto- Optical Beam Splitter

Ammonium persulfate

Basal Ganglia

basic Helix loop Helix

Brain lipid binding protein

Bone Morphogenetic Protein

Base pair

5'-Bromo-2'-deoxyuridine

Bovine Serum Albumin

Corpus Callosum

complementary DNA

Caudal Ganglionic Eminence

complete Hank's Balanced Salt Solution

Cytomegalovirus

Central nervous system

Ciliary Neurotrophic Factor

Cortical Plate

Counts per minute

Cajal-Retzius

Cardiotrophin-1

4',6-Diamidino-2-phenylindole

Deep layer

Dulbecco's Modified Eagle Medium

Dimethylsulfoxide

Deoxyribonucleic acid

Deoxynucleotide triphosphate

Dithiothreitol

Embryonic day

Ethylenediamin-N,N,N',N'-tetra acetic acid 


\begin{tabular}{|c|c|}
\hline EGF & Epidermal Growth Factor \\
\hline EGFR & Epidermal Growth Factor Receptor \\
\hline EMT & Epithelial-Mesenchymal Transition \\
\hline ERK & Extracellular Regulated Kinase \\
\hline $\mathrm{EtOH}$ & Ethanol \\
\hline FGF & Fibroblast Growth Factor \\
\hline FGFR & Fibroblast Growth Factor Receptor \\
\hline Fzd & Frizzled \\
\hline GABA & $\gamma$-amino butyric acid \\
\hline GAPDH & Glyceraldehyde-3-phosphate dehydrogenase \\
\hline GFAP & Glial Fibrillary Acidic Protein \\
\hline GFP & Green Fluorescent Protein \\
\hline HBSS & Hank's Balanced Salt Solution \\
\hline HCL & Hydrochloric acid \\
\hline HD & Homeodomain \\
\hline HEPES & 2-[4-(2-Hydroxyethyl)-1-piperazine]-ethanesulfonic acid \\
\hline HRP & Horseradish Peroxidase \\
\hline $\mathrm{IHC}$ & Immunohistochemistry \\
\hline IL & Interleukin \\
\hline IPC & Intermediate Progenitor Cell \\
\hline IRES & Internal Ribosome Entry Site \\
\hline ISH & In situ hybridization \\
\hline $\mathrm{IZ}$ & Intermediate Zone \\
\hline JAK & Janus Kinases \\
\hline JNK & c-Jun N-terminal Kinase \\
\hline $\mathrm{kDa}$ & kiloDalton \\
\hline LGE & Lateral Ganglionic Eminence \\
\hline LB & Luria-Bertani \\
\hline LIF & Leukemia Inhibitory Factor \\
\hline $\operatorname{loxP}$ & locus of crossover $\mathrm{x}$ in $\mathrm{P} 1$ \\
\hline MEK & Mitogen-activated Protein Kinase Kinase \\
\hline MGE & Medial Ganglionic Eminence \\
\hline $\mathrm{mM}$ & milliMolar \\
\hline mRNA & messenger RNA \\
\hline
\end{tabular}


MZ Marginal Zone

$\mathrm{NaCl} \quad$ Sodium chloride

NEP Neuroepithelium

NF-1 Nuclear factor-1

Ngn2 Neurogenin2

NICD Activated Notch intracellular domain

NT3 Neurotrophin-3

$\mathrm{o} / \mathrm{n} \quad$ overnight

PAGE Polyacrylamide Gel Electrophoresis

PBS Phosphate-Buffered Saline

PCNA Proliferating cell nuclear antigen

PCR Polymerase Chain Reaction

PFA Paraformaldehyde

PH3 Phosphorylated Histone H3

PK Proteinase K

PKC Protein Kinase C

PP Preplate

pVim phospho- Vimentin

RNA Ribonucleic acid

RNase Ribonuclease

rpm Revolutions per minute

RT Room Temperature

RTK Receptor tyrosine kinase

SBD Smad-binding domain

SCM Slice Culture Medium

SDS Sodium Dodecyl Sulfate

SEM Standard error of mean

Sfrp Secreted Frizzled-Related Protein

Shh Sonic hedgehog

Sip1 Smad-interacting Protein 1

SP Subplate

SQ-PCR Semi- quantitative PCR

SSC Sodium chloride-Sodium citrate

STAT Signal Transducer and Activator of Transcription 
STE Sodium chloride-Tris-EDTA

SVZ Subventricular Zone

TAE Tris-Acetate-EDTA

TAQ Thermophilus aquaticus

TBS Tris-Buffered Saline

TE Tris-EDTA

TEMED N,N,N',N'-tetramethyl ethylendiamine

TGF- $\beta \quad$ Transforming Growth Factor- $\beta$

Tris Tris (hydroxymethyl)-amino methane

TrK Tyrosine receptor Kinase

TSA Tyramide Signal Amplification

TUNEL Terminal deoxynucleotidyl Transferase Biotin-dUTP Nick End Labeling

Tween 20 Polyoxyethylene sorbitan monolaurate

UL Upper layer

UTP Uridine Triphosphate

VZ Ventricular Zone

WB Western Blot

Wt Wildtype 


\section{ABSTRACT}

The transcriptional repressor Sip1, or Smad- interacting protein 1, has been shown to play a key role in early neurodevelopment. Mowat- Wilson Syndrome in humans is associated with mutations in Sip1. In the developing mouse cerebral cortex, it is expressed mostly in the differentiating field, and weakly in the proliferative zones. We found that stage and tissue specific ablation of Sip1 leads to several defects in the development of the neocortex. Deletion of Sip1 in cortical progenitors as well as exclusively in postmitotic cortical plate neurons, led to a reduction in the size of deep layer neuronal populations and precocious generation of cortical layers 2-5. Further studies showed that while the onset of neurogenesis is unaffected, this process ends prematurely in conditional Sip1 mutants. We also observed an increase in astrocyte production, and traced its origin to increased and ectopic proliferation of astrocytic progenitors at E17.5, and premature specification of glial precursors at E16.5. Furthermore, elevated proliferation in the cingulate cortex continued until early postnatal stages in the mutant. Our data suggest a possible non- cell autonomous effect of Sip1 on the specification of deep layer versus upper layer neuronal fate, and neuronal versus astrocytic fate of progenitors in the germinal zone, at early and late stages of development, respectively. Comparison of gene expression profile between wildtype and Sip1 conditional knockouts enabled us to identify the growth factors, Neurotrophin-3 (NT3) and Fgf9 as putative downstream targets of Sip1 in the cortex. During early corticogenesis, ablation of Sip1 induces interplay of NT3 and other as yet unconfirmed factor(s) leading to a premature switch in the fate of cortical neuronal precursors. Interestingly, treatment of cortical slices with Fgf9 in vitro led to enhanced production of Olig2 expressing glial precursors at the germinal zone, an effect that recapitulates the phenotype of a Sip1- deficient neocortex. Our data indicate that lack of Sip1 in the cortical plate triggers an Fgf9- mediated signaling cascade back to the germinal zone, possibly via interaction with $\mathrm{Fgfr} 2 / 3$ on radial glial processes, to influence the onset of astrocytic specification. Altogether, our work provides the first evidence of neuron to progenitor feedback signaling in the neocortex, to regulate the fate of uncommitted precursors and ensure production of appropriate numbers of different neurons and glia. Moreover, we have established Sip1 as a key mediator of this mechanism by its negative regulation of expression of certain signaling molecules. 


\section{INTRODUCTION}

A complex interplay of transcription factors and signaling molecules underlies the development of a functional neocortex. A precisely regulated sequence of differential gene expression ensures normal production of the plethora of neurons and glia that make up the cortex.

Specification of the neuroectoderm within a mass of ectodermal cells of the neural plate marks the first step towards induction of the telencephalon. Invagination of the neural plate (presumptive CNS) in response to cues from the underlying mesoderm, followed by closure of the neural folds, results in the formation of a neural tube whose rostralmost region undergoes morphogenesis into the forebrain. While the dorsal neural tube becomes a source of BMPs (Bone Morphogenetic Proteins), the other half uses Shh (Sonic Hedgehog) to develop ventral telencephalic structures. An organizer at the rostral margin of the neural tube, called the anterior neural ridge, ensures induction of telencephalonspecific gene expression. The forebrain subdivides into two cerebral hemispheres, possibly in correlation with formation of the dorsal midline roof plate. Subsequently, each telencephalic half gets specified into pallial (dorsal) and subpallial (ventral) structures. While the pallium includes the medially- located hippocampus and the dorso- laterally situated neocortex, the subpallium comprises the ganglionic eminences. Mutation studies in mice have repeatedly shown that dorsal specification of the cortex involves suppression of ventral fates. At the molecular level, we know that an interplay of BMP- and Wntmediated signaling originating from centres such as the roof plate and the cortical hem, mediates development of dorsal telencephalic structures (reviewed in (Monuki and Walsh, 2001; Wilson and Rubenstein, 2000).

\subsection{The developing neocortex}

During embryogenesis, the neocortex can be broadly divided into the following layers- 1 . The germinal zones, which include the apically situated ventricular zone (VZ), and the basally positioned sub-ventricular zone (SVZ), are the hubs of cortical progenitors. While the $\mathrm{VZ}$ is the earliest layer of progenitors to emerge in the neocortex, non-terminal symmetric divisions of some of its cells subsequently give rise to the SVZ; 2 . Intermediate 

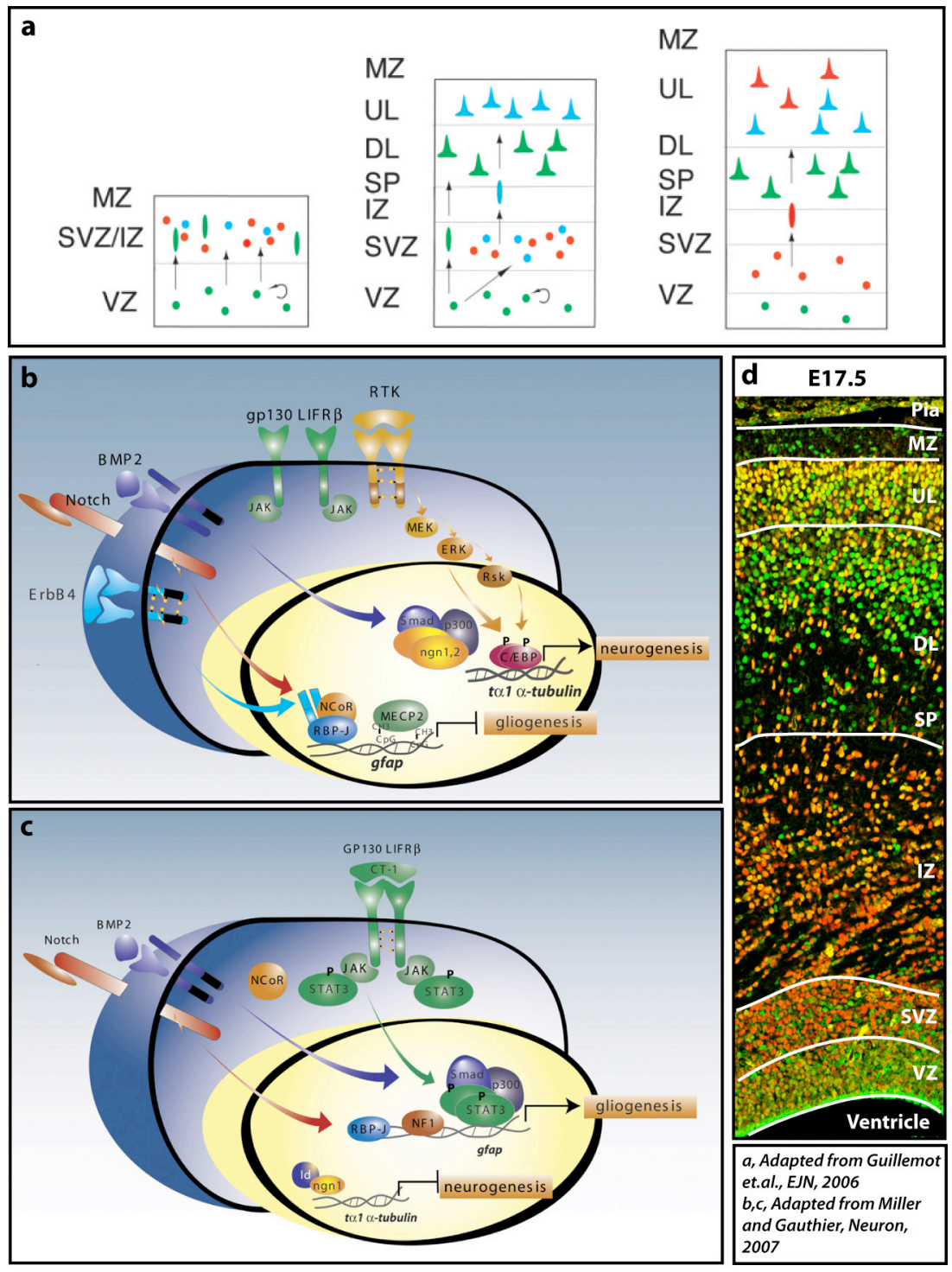

Fig1. Corticogenesis involves a temporally specified sequence of production of neurons and glia. Early in development, a series of proliferative cell divisions expand the pool of progenitors residing in the ventricular zone (VZ), and steadily generate a second geminal zone, called the subventricular zone (SVZ) (a). Differentiative cell divisions in the VZ primarily give rise to deep layer (DL) (Layers 5-6) neurons that migrate radially towards the pial surface into the developing cortical plate (a). Subsequently, upper layer (UL) (Layers2-4) neurons are generated predominantly from SVZ progenitors; these neurons migrate beyond the earlier born DL neurons and occupy the cortical layers immediately below the marginal zone (MZ) (a) (Guillemot et al., 2006). During the neurogenic period of cortical development, bHLH factors such as Ngn2, neurotrophin-RTK-MEK-ERK pathway, and BMP2- mediated signaling cooperate to promote neuronal differentiation. Simultaneously, gliogenic genes are repressed by promoter methylation and Notch-, Neuregulin-ErbB4 mediated transcriptional silencing (b) (Miller and Gauthier, 2007). During the gliogenic period, cytokine (CT-1)- mediated Jak/Stat signaling, activation of Notch- and BMP2- pathways, and proastrocytic transcription factors such as NF-1, ensure astrocyte production (c) (Miller and Gauthier, 2007). 
A cross- section through the developing neocortex at E17.5 shows the arrangement of the VZ, SVZ, intermediate zone (IZ), UL and DL (together called the cortical plate), and the MZ within one radial unit from the ventricular to the pial surface (d).

zone (IZ), located basal to the SVZ, is comprised of young cortical neurons migrating towards their final destination along a route perpendicular to the ventricular surface. At the SVZ/IZ junction, interneurons migrating tangentially from the ganglionic eminences, can be seen; 3. Cortical plate (CP), located basal to the IZ, comprises young post-migratory postmitotic neurons undergoing terminal differentiation and maturation. The $\mathrm{CP}$ can be further subdivided into upper layers (UL, layers 2-4) and deep layers (DL, layers 5 and 6); these cytoarchitecturally and functionally different cells make up the mature adult cortex; 4. Marginal zone (MZ), located below the layer of connective tissue called pia mater, is populated by Reelin secreting Cajal- Retzius cells (described below). A second stream of tangentially migrating interneurons can be observed below the MZ.

\subsection{Cortical progenitors}

\subsubsection{Neuroepithelial cells/Radial glia}

Corticogenesis begins with a highly polarised pseudostratified neuroepithelium lining the lateral ventricle. Many of these cells subsequently transform into radial glial cells that, directly or indirectly, generate most neurons of the cortex. In contrast to the pluripotent neuroepithelial cells, individual radial glial cells are more fate- restricted, as shown by in vivo recombination- based fate mapping (Gotz and Huttner, 2005). Retroviral labeling followed by clonal analysis has indicated that the radial glial population is comprised of several sublineages, some of which might be neuronal- type specific (Kriegstein and Gotz, 2003; McCarthy et al., 2001). For a long time, these cells were believed to be distinct from neuronal precursors of the neocortex. Retroviral tracing provided the first direct evidence of radial glia generating neurons in vivo during the peak neurogenic period in the neocortex (Noctor et al., 2001). Radial glial cells possess a basally oriented process that extends towards the pial surface, while the nucleus lies in the ventricular zone (VZ). Division of these cells is accompanied by a characteristic movement of the nuclei away from the apical surface during S-phase, and towards the ventricle during G2 phase, so that the M-phase of cell cycle is completed at the apical surface. This process is referred to as 
interkinetic nuclear migration, first described about 70 years ago (Sauer and Walker, 1959; Tamai et al., 2007). Expression of markers like Nestin, RC1/2, GLAST, Blbp, GFAP, tenascin- $\mathrm{C}$ and glycogen granules (Malatesta et al., 2003) characterises the radial glial progenitors.

\subsubsection{Basal Progenitors}

At least three independent research groups used time- lapse microscopy to demonstrate the existence of a second type of cortical progenitors that lie basal to the VZ in a region called the subventricular zone (SVZ). These arise from asymmetric divisions of radial glial cells where one daughter cell moves to the SVZ and divides again. The majority of SVZ cells undergo symmetric terminal divisions to generate two daughter neurons that then migrate into the cortical plate, while a small percentage undergo self- renewing divisions. These SVZ progenitors are also called basal progenitors or intermediate progenitors, differing subtly in marker expression and cell fate. For instance, although SVZ progenitors have been shown to be the major source of cortical astrocytes (Levison and Goldman, 1997; Parnavelas, 1999), Tbr2 expressing intermediate progenitors have never been reported to generate any glial cell (Arnold et al., 2008; Kowalczyk et al., 2009; Noctor et al., 2004). Although basal progenitors coexist with the apically dividing VZ cells from the onset of neurogenesis in the cortex, as development proceeds, they tend to outnumber the latter and become the major source of cortical neurons as well as astrocytes. At the end of neurogenesis, radial glial cells undergo one final asymmetric division to give rise to, in most cases, a basal progenitor and an astrocyte (Haubensak et al., 2004; Miyata et al., 2004; Noctor et al., 2004). Evolutionarily, the expansion of the SVZ in relation to the VZ has been proposed as a way of producing more neurons per unit surface area of the ventricular lumen, and thereby, for enlargement of the cortex (Haubensak et al., 2004).

\subsection{Cell cycle in Corticogenesis}

Proper formation of the cortex is also controlled by several cell cycle parameters. It is known that throughout the 6- day period of neuronogenesis in the mouse cortex, the length of the cell cycle increases from $8 \mathrm{hrs}$ to $18 \mathrm{hrs}$, all the while maintaining constant duration of S- phase (3-4hrs) and G2+M phase (2hrs) (Takahashi et al., 1995). In total, the cortex manages to complete 11 cell cycles within the 6-day window (Cai et al., 2002). The 
correlation between the length of the cell cycle and neurogenesis was convincingly demonstrated through a series of experiments. Firstly, it was shown that lengthening of cell cycle by using inhibitors of cyclin- dependent kinases leads to premature neurogenesis (Calegari and Huttner, 2003). Secondly, Tis21 expressing neurogenic VZ cells were shown to have longer cell cycles than their proliferative counterparts (Calegari et al., 2005). Three types of cell divisions contribute to corticogenesis- symmetric nonterminal (expansion of progenitors), asymmetric (contributes to maintenance of progenitor pool as well as neurogenesis), and symmetric terminal (contributes to neurogenesis). Retroviral lineage tracing has shown that all three modes of division occur throughout the period of neurogenesis in mice; their relative proportions however, keep changing (Cai et al., 2002). Rate of cell cycle exit is another crucial factor, as was shown in B-catenin overexpressing mice, where increased mitotic reentry leads to an expansion of progenitor pool and consequently, enlarged cortical surface area (Chenn and Walsh, 2002). At earlier stages of corticogenesis, most cell divisions are proliferative and result in increasing the precursor pool, while as development proceeds, the fraction of differentiative cell divisions progressively increases leading to enhanced rate of mitotic exit and consequently, neurogenesis.

\subsubsection{Regulation of neuron number}

The number of neurons generated in the cortex depends on three main factors: the proportion of symmetric versus asymmetric divisions, the length of the cell cycle, and the rate of mitotic exit (Dehay and Kennedy, 2007). While the molecular mechanisms regulating the ratio of proliferative versus differentiative divisions are not clear, it is highly plausible that an interplay of both cell- intrinsic as well as cell- extrinsic programs is involved. As mentioned above, lengthening of G1 phase bears a striking correlation with differentiation, and response to neuronal- determination signals (reviewed in (Dehay and Kennedy, 2007).

\subsection{Sequential generation of neurons and astrocytes in the cortex}

In the developing cerebral cortex, neurogenesis is initiated rostrolaterally and progresses along the caudomedial axis of the epithelium (Bayer and Altman, 1991). 
The first layer of postmitotic cells that appear in the cortex form the preplate; this layer includes the Cajal- Retzius cells migrating into the cortex from the cortical hem and the ventral pallium, among other sources. Presently, neurons born in the neocortical ventricular zone as a consequence of asymmetric cell divisions, migrate toward the pial surface and split the preplate into the marginal zone and the subplate. While the $\mathrm{MZ}$ is populated by Reelin secreting Cajal- Retzius cells migrating into the cortex from the cortical hem and the ventral pallium, among other sources (Bielle et al., 2005), the subplate is essential for proper establishment of the thalamocortical circuitry (Hevner et al., 2002; HoerderSuabedissen et al., 2008). Subsequently born neurons migrate over the subplate to form the six layers of the cortex in a characteristic inside first- outside last pattern. Neurons of deep layers 6 and 5 are generated prior to those of upper layers 4, 3 and 2, that end up occupying more superficial positions within the cortical plate. The entire sequence is temporally specified in such a way that the date of birth of a neuron can be used to accurately predict its laminar fate (McConnell, 1988; (Bayer and Altman, 1991).

Both in vivo as well as in vitro, the generation of cortical cell types follows a precise sequence, such that neurons are born first (E12- E17) followed by astrocytes (predominantly E17- P2), and finally oligodendrocytes that are only seen postnatally. Time- lapse microscopy based clonal analysis as well as retroviral lineage tracing studies have shown that single cortical precursors can give rise to first, early- born neurons, then late- born neurons, then astrocytes, and finally oligodendrocytes. The fact that the fate of late precursors cannot be reversed on exposure to early stage microenvironment although early precursors can progress to a later fate when transplanted to a late germinal zone, led to the belief that cortical precursors not only change their competency over time but also become progressively lineage- restricted. Further corroborating the above hypothesis, coculture paradigms showed that when early embryonic precursors are cultured on embryonic slices, they generate neurons, when cultured on postnatal slices, they generate astrocytes. On the other hand, when postnatal precursors are cultured on cortical slices, irrespective of the age of the slice, they always generate glia. There are contrasting theories on whether the information regarding the timing of neuro-/gliogenesis is exclusively dependent on intrinsic programs within progenitors or is significantly influenced by the extrinsic environment as well (Barnabe-Heider et al., 2005; Desai and McConnell, 2000; McCarthy et al., 2001; Miller and Gauthier, 2007; Morrow et al., 2001; Qian et al., 2000; Reid et al., 1995; Shen et al., 2006). There is limited knowledge about the role of 
extracellular signals in determination of progenitor fate. Discussed further are specific reports on the function of CP to VZ feedback signaling.

\subsection{Molecular control of Neurogenesis in the cortex}

Early neurogenesis relies largely on receptor tyrosine kinase (RTK) mediated activation of MEK-ERK signaling cascade. The significance of MEK-ERK mediated cascade in transducing growth factor signals, including those of neurotrophins, to neuron- specific gene transcription is well established (Barnabe-Heider and Miller, 2003; Menard et al., 2002; Paquin et al., 2005).

The axonal connectivity of neurons populating the cortex was revealed by retrograde dye tracing experiments- layer 6 neurons primarily extend subcortical projections to the thalamus, while layer 5 neurons extend axons towards subcerebral targets such as spinal cord, pons and tectum; on the other hand, layer 2-4 neurons project both ipsilaterally as well as contralaterally within the cortex, leading to the formation of tracts such as corpus callosum (Fishell and Hanashima, 2008; Leone et al., 2008).

Morphogenesis of the cerebral cortex involves an interplay between Pax6 and Emx2. The opposing rostrolateral versus caudomedial gradient of Pax6 and Emx2 expression is not only required for proper arealisation but also for specifying the identity of the dorsal telencephalon. Loss of Emx2 function causes expansion of anterior-lateral cortical regions (eg., somatosensory cortex), while loss of Pax6 causes expansion of posterior-medial territories (eg., visual cortex). On the other hand, loss of both Emx2 and Pax6 leads to misspecification of the dorsal telencephalon into the ventrally located ganglionic eminences (Bishop et al., 2000; Mallamaci et al., 2000; Muzio et al., 2002; Muzio and Mallamaci, 2003). Subsequently, commitment of cortical progenitors towards a neuronal fate requires expression of proneural genes, especially Ngn2 and Ngn1, loss of which leads to misspecification of glutamatergic neuronal precursors to GABAergic neuronal precursors within the early stage cortex. Loss of both Ngn2 and Mash1 leads to enhanced astrocytogenesis at the expense of neurogenesis. The identity of late-born cortical glutamatergic neurons is specified by a different mechanism, possibly mediated via Pax6 (Guillemot et al., 2006; Nieto et al., 2001; Schuurmans et al., 2004). Once specified, a neuronal differentiation program is initiated via expression of genes like NeuroD1/D2, Math2, Tbr1 and Tbr2. The advent of molecular markers as a tool to study cortical layering 
was perhaps the most significant development in the study of mammalian corticogenesis. Some of these markers have been studied extensively and shown to be essential and indispensable for normal cortex formation. These include transcription factors such as, 1 . Tbr2, an SVZ marker required for basal proliferation and generation of upper layer neurons (Arnold et al., 2008); 2. Tbr1, a layer 6 marker, is essential for proper neuronal migration, subplate differentiation, and axonal connectivity (Hevner et al., 2001); 3. Ctip2, a layer 5 marker, is essential for development of corticospinal motor neurons (Arlotta et al., 2005); 4. Satb2, a layer 2-4 marker, is required for specification of callosal projection neurons (Alcamo et al., 2008; Britanova et al., 2008); 5. Brn1/2, a layer 2-4 marker, is essential for upper layer neuron production and migration (Sugitani et al., 2002).

These and other transcription factors are essential not only to specify a certain lineage at the precursor level, but some also to maintain neuronal identity at the postmitotic level. It was shown recently that absence of Satb2 in callosally projecting layer 2-4 neurons causes them to switch fate to Ctip2+ subcortical projection neurons (Alcamo et al., 2008; Britanova et al., 2008). Differences in the onset and level of expression of Tbr1, Fezf2, Sox5, Ctip2 and Satb2, leading to mutual activation/repression, clearly control the subcortical versus callosal projection neuron identity (Leone et al., 2008).

\subsection{Molecular control of Astrogliogenesis in the cortex}

Cortical stem cells can be coerced into astrocytic differentiation in the presence of growth factors such as platelet- derived growth factor (PDGF), ciliary neurotrophic factor (CNTF), interleukin-6 (IL6), leukemia inhibitory factor (LIF), glial growth factor 2 (GGF2), epidermal growth factor (EGF), Fibroblast growth factor 2 (Fgf2) and bone morphogenetic proteins (BMPs). Interestingly, CNTF, LIF and IL6 are known to induce gliogenesis in vitro although neither of them is expressed endogenously in the developing brain.

There is an intricate network of crosstalk between various growth factors and transcription factors on the way to astrocytic differentiation, such that cortical response to the same factor differs substantially at different stages of development. BMPs, for instance, induce neurogenesis in early precursors and gliogenesis in late precursors, due to the fact that early precursors express high levels of the bHLH transcription factor Ngn2 making them non- responsive to gliogenic cues (Gross et al., 1996; Li et al., 1998; Sun et al., 2001). 
Similarly, LIF, Fgf2 and activation of the Notch- Delta pathway can trigger an astroglial fate only in late and not early progenitors, where Notch- mediated signaling is responsible for maintaining cells in a proliferative state instead (Chambers et al., 2001; Ge et al., 2002; Molne et al., 2000; Song and Ghosh, 2004; Tanigaki et al., 2001; Viti et al., 2003). Altogether, this suggests that late progenitors are competent to respond appropriately to gliogenic cues, either due to downregulation of Ngn1 expression or upregulation of EGF receptors (EGFR), among other possible reasons. Ngn1 was shown to specifically repress glial- specific gene expression by 1 . Suppressing LIF/CNTF induced phosphorylation of STATs, thereby suppressing Jak/Stat signaling; and, 2. Sequestering transcriptional complexes containing activated Smads away from STATs (Sun et al., 2001). The increase in EGFR expression during later development renders progenitors competent to respond to LIF/CNTF as an astrocytic signal, due to their modulation of STAT3 expression (Viti et al., 2003). Intracellularly, activation of the JAK- STAT pathway is crucial to the astrogliogenic machinery. Over the years, several lines of evidence have established this fact. First of all, LIF and CNTF promote astrocyte differentiation by activating JAKSTAT pathway; mutation of LIF, its receptors or STATs leads to impaired astrocytogenesis (Bonni et al., 1997; Koblar et al., 1998; Nakashima et al., 1999a). Secondly, BMP2, Fgf2 and Notch signaling mediated astrocytogenesis are more effective in combination with activated JAK-STAT pathway (Ge et al., 2002; Nakashima et al., 1999b; Song and Ghosh, 2004; Sun et al., 2001). Moreover, anti- gliogenic factors like Ngn1 and Ngn2 inhibit astrocytogenesis by suppressing activation of the JAK-STAT pathway, while EGFRs act by modulating STAT expression and activation (Sun et al., 2001; Viti et al., 2003). Furthermore, proneural genes seem to be critical for regulating the timing of gliogenesis. This is evident from the premature gliogenesis observed in Ngn2/Mash1 double knockouts and in Notch overexpressing cortices that downregulate expression of bHLH factors (Morrison et al., 2000; Nieto et al., 2001). Finally, the role of JAK-STAT signaling in gliogenesis is strengthened by the reported positive autoregulation of components of this pathway by activated STAT1/3 (He et al., 2005). 


\subsection{Neuronal migration}

\subsubsection{Radial migration}

Radial migration of young neurons in the cortex takes place primarily via two modes:locomotion, and somal translocation (Kriegstein and Noctor, 2004; Nadarajah et al., 2001; Rakic, 2007). Locomotion involves the movement of neurons over a radial glial process that they use as a scaffold. A complex molecular machinery is involved in regulating the individual steps, starting from extension of the leading process to attachment to the RG fiber to the rate of migration to detachment and cessation of migration. On the other hand, cells undergoing nuclear translocation or nucleokinesis do not need a glial support; they extend a leading process attached to the pial surface through which the nucleus moves to its destination. Somal translocation seems to be the preferred mode of radial migration for neurons born at early stages of neurogenesis when the distances are shorter, while radial glia- guided locomotion is more commonly detected at later stages when distances increase (Nadarajah et al., 2003; Nadarajah et al., 2001; Tissir and Goffinet, 2003). Retroviral lineage analysis and time- lapse microscopy has shown that neurons born in the neocortex often disperse tangentially to neighboring radial units of the cortex (Kriegstein and Noctor, 2004; Walsh and Cepko, 1993). Interestingly, VZ progenitors have also been shown to disperse across radial units(Fishell et al., 1993; Wilkie et al., 2004).

At the molecular level, two major signaling pathways are known so far to regulate cortical migration: 1. Reelin, a secreted glycoprotein, is expressed by Cajal- Retzius cells in the marginal zone. The latter are amongst the earliest cell types to be generated in the cortex from sources such as the cortical hem and the pallial- subpallial boundary(Bielle et al., 2005; Takiguchi-Hayashi et al., 2004). Mutations in Reelin, its receptors, Vldlr and ApoER2, and the intracellular adaptor protein Dab-1, all leads to lack of preplate splitting and improper migration of neurons leading to a radial intermixing of the different neuronal types (Howell et al., 1997; Sheldon et al., 1997; Sheppard and Pearlman, 1997; Tissir and Goffinet, 2003; Trommsdorff et al., 1999). It was proposed long ago that Reelin provides a stop signal to migrating neurons and instructs them to terminate migration either by affecting nucleokinesis or by detaching neurons from the radial glial scaffold. On the other hand, it is also possible that Reelin acts as an attractant for neurons to migrate past the subplate. However, both these hypotheses are doubtful in light of the observation that 
ectopic expression of Reelin in the VZ, which is equipped with the appropriate response machinery, is sufficient to partially, but significantly, rescue the reeler phenotype, suggesting that the physical source of Reelin in the cortex is not important for preplate splitting but only for proper lamination. Altogether, it seems that neurons respond differentially to Reelin and that the latter acts through a complicated mechanism (Magdaleno et al., 2002; Tissir and Goffinet, 2003). 2. p35/p39/Cdk5- mediated mechanism seems to be essential for migration later during corticogenesis. This is evident from the fact that in single mutants for Cdk5 and p35 and in double mutants for p35 and p39, the preplate does get split by early- born neurons, however, the later born neurons fail to cross the subplate and end up beneath this layer. Consequently, the subplate is positioned superficially in these mutants with a thin cortical plate separating it from the marginal zone (Gilmore et al., 1998; Ko et al., 2001; Kwon and Tsai, 1998).

\subsubsection{Tangential migration}

Retroviral lineage tracing, cell transplantation experiments as well as analysis of mutant animals has proved that the medial, lateral and caudal ganglionic eminences (MGE/LGE/CGE) are the primary source of cortical interneurons in rodents. Cortical interneurons born in the ganglionic eminences migrate tangentially into the dorsal cortex through two major routes: the intermediate zone (IZ)- subventricular zone (SVZ), and the marginal zone $(\mathrm{MZ})$. After entering the neocortex these neurons often turn and migrate radially into defined layers within the cortical plate. Interestingly, most interneuron types are born contemporaneously with the pyramidal neurons that occupy the same cortical layer. In other words, interneurons migrate tangentially to occupy the same cortical layer that is occupied by radially migrating pyramidal neurons born at the same time in the neocortex (reviewed in (Kriegstein and Noctor, 2004; Xu et al., 2003).

\subsection{Smad- interacting protein 1 (Sip1)}

Sip1 (also called Zfhx1b/ZEB2) was identified as an interacting partner of receptorregulated TGFß/BMP Smads in a yeast- two hybrid screen using E12.5 mouse cDNA library (Verschueren et al., 1999). It is primarily a transcriptional repressor containing two zinc- finger domains, one at the $\mathrm{N}$ - terminal, and one at the $\mathrm{C}$ - terminal; it binds, as a monomer, to bipartite $5^{\prime}-\mathrm{CACCT}(\mathrm{G})$ sequences separated by varying distances ( $44 \mathrm{bp}$ in 
case of E-Cadherin) on the promoters of its target genes (Remacle et al., 1999). In Xenopus, besides the Smad proteins, Sip1 also forms complexes with the co- repressor CtBP and the co- activators p300/CBP associated factor (van Grunsven et al., 2003; van Grunsven et al., 2006). One of the first genes whose expression was shown to be repressed by Sip1 in the mammalian system, is the cell- cell adhesion molecule E-cadherin. Loss of E-cadherin is associated with several instances of tumor progression, invasion and metastasis. Sip1 was also shown to directly downregulate the expression of genes coding for several cell junction proteins, and thus induce epithelial mesenchymal transition (EMT) (Comijn et al., 2001; Vandewalle et al., 2005). All these studies, however, were performed in vitro. In vivo, mutations in the human Sip1 gene are associated with mental retardation, hypertelorism, cranio- facial dysmorphology, and microcephaly, a condition called MowatWilson syndrome, a variation of Hirschprung Disease (Wakamatsu et al., 2001; Zweier et al., 2002). Homozygous Sip1 mouse mutants die at E9.5; when analysed earlier, they exhibited failure to close the neural tube, lack of vagal neural crest, defective migration of cranial neural crest cells, and reduction of early neuroectodermal marker Sox2. This implies that Sip1 is essential for proper specification of the neural plate (Van de Putte et al., 2003). Cortex- specific conditional knockout of Sip1, on the other hand, leads to a progressively degenerating hippocampal formation, possibly due to upregulation of Wnt antagonist Sfrp1 and consequently, reduced activity of the JNK- mediated non- canonical Wnt pathway (Miquelajauregui et al., 2007). In Xenopus, Sip1 is essential at first, for specifying neural tissue in the anterior region of the developing embryo, and secondly, for neural differentiation, primarily via inhibition of the BMP pathway (Eisaki et al., 2000; Nitta et al., 2004). Recently, Sip1 was shown to play a role in neural patterning in zebrafish as well (Delalande et al., 2008). Additionally, Sip1 has also been reported to act as a transcriptional activator in vivo (Yoshimoto et al., 2005).

In a subtractive hybridization based screening done in our lab, Sip1 was identified as a gene expressed at much higher levels at E15.5 than at E13.5 in mice. Since E15.5 is the peak of production of upper layer neurons in the neocortex, we speculated that Sip1 might have a selective function in specifying such a fate. Moreover, studies in Xenopus and of Sip1 total knockouts in mice clearly suggest an important function of this transcription factor in neural development. In this study, we set out to explore the functions of Sip1 in cortical lamination, neuronal migration and gliogenesis, using cortex- specific conditional mutants. Through our work, we identified Sip1 as a critical regulator of the sequential 
generation of cortical cell types. Furthermore, we have shown that Sip1 acts non- cell autonomously through Fgf9 and possibly NT3- mediated molecular mechanisms to negatively regulate premature fate switch of cortical precursors during development. 


\section{MATERIALS AND METHODS}

\subsection{Mouse mutants}

\subsubsection{Conditional Sip1 knockout}

Mice carrying floxed Sip1 allele(s) (Higashi et al., 2002) were crossed with those carrying Cre recombinase under the control of a tissue/ stage- specific promoter (Cre lines). The following Cre lines were used:- Emx1-Cre (Goebbels et al., 2006; Gorski et al., 2002) and Nex-Cre (Goebbels et al., 2006). The conditional mutants contained 2 copies of floxed Sip1 allele alongwith atleast one Cre allele, and are designated as Sip1fl/flEmx ${ }^{\mathrm{Cre}}$ and Sip1fl/flNex ${ }^{\mathrm{Cre}}$. Mice with any of the following genotypes- Sip1wt/wt, Sip1wt/wtEmx ${ }^{\mathrm{Cre}}$, Sip1wt/wtNex ${ }^{\mathrm{Cre}}$, Sip1fl/wt, Sip1fl/wtEmx ${ }^{\mathrm{Cre}}$, Sip1fl/wtNex ${ }^{\mathrm{Cre}}$, were used as control littermates, designated as $\mathrm{Wt}($ (wildtype). We did not find any phenotypic differences between Sip1wt/wtEmx ${ }^{\mathrm{Cre}}$ (or $\mathrm{Nex}^{\mathrm{Cre}}$ ) and Sip1fl/wtEmx ${ }^{\mathrm{Cre}}$ (or $\mathrm{Nex}^{\mathrm{Cre}}$ ). As part of the breeding strategy, heterozygous mice (Sip1fl/wtEmx ${ }^{\mathrm{Cre}}$ (or Nex $\left.{ }^{\mathrm{Cre}}\right)$ ) were backcrossed with C57B6 wildtype mice, and the F1 generation was interbred to get Sip1 conditional knockouts for experimental studies. In some experiments involving Sip1fl/flNex ${ }^{\mathrm{Cre}}$ and control littermates, mice lacking one functional Sfrp1 allele were used. As discussed later, we observed no phenotypic differences between wildtype and Sfrp1+/-, and between Sip1fl/flEmx ${ }^{\mathrm{Cre} /}$ Sip1f1/flNex ${ }^{\mathrm{Cre}}$ and Sip1f1/flEmx ${ }^{\mathrm{Cre}}$ Sfrp1+/-/ Sip1f1/flNex ${ }^{\mathrm{Cre}}$ Sfrp1+/embryos. Hence, Sfrp1+/- was considered as a control genotype.

\subsubsection{Sip1-Sfrp1 double mutants}

To generate Sip1-Sfrp1 double mutants, heterozygotes (Sip1fl/wtEmx ${ }^{\mathrm{Cre}}$ (or Nex ${ }^{\mathrm{Cre}}$ )) were bred with Sfrp1 total knockouts (Sfrp1-/-) (Bodine et al., 2004). The latter were generated by deleting exon1, which codes for most of the protein including the Cysteine rich domain that is responsible for sequestering Wnts away from their receptors. The mutant allele contains a LacZ coding sequence instead of exon1. The F1 generation was then interbred to obtain double mutants- Sip1fl/flEmx ${ }^{\mathrm{Cre}}$ (or Nex ${ }^{\mathrm{Cre}}$ ) Sfrp1-/-. Both Sfrp1+/+ as well as Sfrp1+/- in combination with any of the control Sip1 genotypes mentioned before, were used as controls in this analysis. 


\subsubsection{Sip1-Fgfr1 double mutants}

To generate Sip1- Fgfr1 double conditional mutants, heterozygotes (Sip1fl/wtEmx ${ }^{\mathrm{Cre}}$ ) were bred with Fgfrlfl/fl (obtained from Dr. Mario Giraldo, University of Goettingen) mice. The F1 generation was interbred to obtain double floxed alleles that would get truncated in the presence of at least one copy of Cre. Therefore, double knockouts have the genotype, Sip1fl/flFgfr1fl/flEmx ${ }^{\mathrm{Cre}}$, while Fgfr11fl/wtEmx ${ }^{\mathrm{Cre}}$, in combination with the control Sip1 genotypes mentioned before, were considered as controls.

\subsubsection{Genotyping}

Tail tissue was digested in $0.3 \mathrm{ml}$ Lysis buffer $(100 \mathrm{mM}$ Tris- $\mathrm{HCl} \mathrm{pH} 8.5,5 \mathrm{mM}$ EDTA, $200 \mathrm{mM} \mathrm{NaCl}, 0.2 \% \mathrm{SDS}, 100 \mu \mathrm{g} / \mathrm{ml}$ Proteinase K) at $55^{\circ} \mathrm{C}$ for $2 \mathrm{hrs}$ to overnight. Strands of hair were removed by centrifugation at $9000 \mathrm{rpm}$ for $10 \mathrm{mins}$. The DNA in the supernatant was precipitated by adding an equal volume of isopropanol, followed by gently mixing and centrifugation at $13000 \mathrm{rpm}$ for $15 \mathrm{mins}$. The DNA precipitate was washed twice in $80 \%$ ethanol, air dried and resuspended in 100 to $200 \mu 1$ sterile distilled water.

All PCR reactions were done in a final volume of $20 \mu \mathrm{l}$ in a mixture prepared according to the following protocol-

10x Buffer (Genecraft) - $2 \mu 1$

$10 \mathrm{mM}$ dNTPs (Invitrogen) - $0.4 \mu \mathrm{l}(20 \mathrm{pmol} / \mathrm{ml})$

$10 \mathrm{nmol} / \mathrm{ml}$ Forward/Reverse primers (IBA) - $0.8 \mu \mathrm{l}(40 \mathrm{pmol} / \mathrm{ml})$ each

TAQ polymerase (Genecraft/USB) - $0.4 \mu \mathrm{l}$ (0.5 units)

Template DNA - $1 \mu 1$

$\mathrm{dd} H 2 \mathrm{O}-13 \mu \mathrm{l}$

The following primer sequences/ reactions were used-

1. Sip1-floxed and wildtype alleles

Forward- 5' TGGACAGGAACTTGCATATGCT 3'

Reverse- 5' GTGGACTCTACATTCTAGATGC 3'

Amplification program-

$94^{\circ} \mathrm{C}-10$ ',

$59^{\circ} \mathrm{C}-20^{\prime}$,

$72^{\circ} \mathrm{C}-40^{\prime}$ 
31 cycles; wildtype allele yields a product of $\sim 450 \mathrm{bp}$ and the floxed Sip1 allele yields a product of $\sim 600 \mathrm{bp}$.

2. Cre allele (for both Emx1-IRES-Cre and Nex-Cre)

Forward- 5' TCGATGCAACGAGTGATGAG 3'

Reverse- 5' TTCGGCTATACGTAACAGGG 3'

Amplification program-

$94^{\circ} \mathrm{C}-10^{\prime \prime}$

$55^{\circ} \mathrm{C}-30^{\prime \prime}$

$72^{\circ} \mathrm{C}-40^{\prime \prime}$

30 cycles; presence of atleast one Cre allele yields a product of $\sim 500 \mathrm{bp}$.

3. Sfrp1 truncated and wildtype allele

For the wildtype allele-

Forward- 5' GGCAGCCCCGACGTCGCCGAGCAAC 3'

Reverse- 5' CCTTGGGGTTAGAGGCTTCCGTGG 3'

For the truncated Sfrp1 allele-

Forward- 5' ACGGCATGGTGCCAATGAATCGTCTG 3'

Reverse- 5' CAAATAATATCGGTGGCCGTGGTGTC 3'

Amplification program-

$95^{\circ} \mathrm{C}-20^{\prime \prime}$

$62^{\circ} \mathrm{C}-30^{\prime \prime}$

$72^{\circ} \mathrm{C}-40^{\prime \prime}$

$95^{\circ} \mathrm{C}-30^{\prime}$

34 cycles; wildtype allele yields a product of $379 \mathrm{bp}$ while the mutant allele generates a 212 bp long PCR product.

4. Fgfr1 wildtype and floxed alleles

Forward- 5' CTAGGCCAGCTGGACCAAAC 3'

Reverse- 5' CATCTTCTCGGTGTTCGTCC 3'

Amplification program-

$94^{\circ} \mathrm{C}-3^{\prime}$

$94^{\circ} \mathrm{C}-30^{\prime}$,

$58^{\circ} \mathrm{C}-30^{\prime \prime}$ 
$72^{\circ} \mathrm{C}-1$ ' $30^{\prime \prime}$

$72^{\circ} \mathrm{C}-5^{\prime}$

37 cycles; wildtype allele yields a product of $\sim 200 \mathrm{bp}$ while the floxed allele yields a product of $\sim 300 \mathrm{bp}$.

All PCR products were analysed by electrophoresis on a 1.2-1.4\% agarose gel at $90-120 \mathrm{~V}$. The products were separated in TAE buffer ((40mMTris- acetate, 1mMEDTA, pH 8) containing $0.5 \mu \mathrm{g} / \mathrm{ml}$ ethidium bromide (Fulka), and were visualised under UV illumination in a gel documentation system. Loading dye consisted of OrangeG dye $(0.2 \%)$ and sucrose (40\%). The size of the PCR products was determined by comparing with standard DNA ladders (Invitrogen) loaded at 200ng/ $\mu 1$.

\subsection{Tissue processing}

The day of vaginal plug was considered embryonic day (E) 0.5 . For assaying proliferation, BrdU (SIGMA) was administered to pregnant females by intraperitoneal injections at a concentration of $100 \mathrm{mg} / \mathrm{kg}$ body weight; the females were sacrificed 1 and $1 / 2$ hrs later. For birthdating experiments, the BrdU injected females were allowed to deliver, and the pups were sacrificed at P2 (postnatal day 2). Pregnant females were sacrificed either by cervical dislocation or in a $\mathrm{CO}_{2}$ chamber, while newborn and P1-P5 pups were sacrificed by decapitation. Brains (E15.5 to P5) or whole heads (E12.5 to E14.5) were dissected out and immersed in 4\% PFA (prepared in PBS) for 4-8hrs (E12.5- E16.5) to overnight (E17.5 and older brains). Mice older than P5 were first perfused with, then immersed overnight in, 4\% PFA, They were then washed in PBS several times. Thereafter, brains were processed in either of two ways depending on the type of sectioning that was required for the experiment- In the first method, they were incubated overnight in $0.9 \% \mathrm{NaCl}$, followed by progressive dehydration through a series of increasing strength of ethanol (50\% to $100 \%)$, followed by isopropanol. They were then incubated in toluol for atleast $6 \mathrm{hrs}$, followed by Paraplast wax (maintained at $60^{\circ} \mathrm{C}$ ) (twice, overnight). Finally they were embedded in the wax within plastic chambers and sectioned into $10 \mu \mathrm{m}$ thick frontal sections using a Microtome (Leica). The sections were mounted on silane- coated slides (Marienfeld Histobond). In the second method, brains were cryoprotected in $15 \%$ followed by $30 \%$ sucrose, and embedded in OCT (TissueTek). They were then sectioned into $10 \mu \mathrm{m}$ thick 
frontal sections using a Cryotome (Leica) and mounted on Superfrost Plus slides (MenzelGläser).

\subsubsection{Perfusion}

Mice older than P5 were perfused with 4\% PFA before immersing in the same solution overnight in order to ensure proper fixation of the tissue. The mice were anesthetized by intraperitoneal administration of Avertin (100-300 $\mu$ l per animal, depending on the age). Subsequently, they were dissected open to expose the chest cavity, the rib cage was cut, followed by the diaphragm. A fine needle connected to a syringe which is in turn connected to a source of cooled PBS was inserted into the left ventricle and held in position; in order to leak out excess solution, a small cut was made in the right auricle. This would ensure circulation of PBS, facilitated by the initial beating of the heart, throughout the body. As a consequence, visceral organs such as the liver can be seen to turn pale in color (as the blood is replaced with PBS). Once this was detected, the PBS was replaced with 4\% PFA and the perfusion was continued for a few minutes until certain prominent signs of penetration of PFA into tissues was detected. The signs would include, movement of the head (in case of young pups), movement of the tail (in juvenile and adult mice) etc. After this, the brain was dissected out (should appear pale and devoid of any blood in the vessels), and immersed in 4\% PFA overnight.

\subsection{Nissl Staining}

Paraffin embedded sections mounted on slides were dewaxed in Histoclear and rehydrated by passing through a descending series of ethanol concentrations. They were washed in $\mathrm{H}_{2} \mathrm{O}$, incubated in $50 \%$ potassium sulfite for $15 \mathrm{mins}$, washed again, and stained in Cresyl violet (1.5\% in acetate buffer) for approx. 20mins. To remove excess stain, the sections were then washed in acetate buffer $(10 \mathrm{mM}$ sodium acetate, $10 \mathrm{mM}$ acetic acid in $\mathrm{H} 2 \mathrm{O})$ for 2 mins followed by Differentiation buffer $(0.14 \%$ glacial acetic acid $)$ until the desired color/intensity was obtained. This was followed by alcohol- assisted rehydration and air drying of slides. Finally, they were mounted in a Xylol- based hydrophobic mounting medium (Eukitt) 


\subsection{Immunohistochemistry}

\subsubsection{Standard protocol (without amplification of signal)}

All paraffin sections were dewaxed in Histoclear and rehydrated by passing through a descending series of ethanol concentrations. After washing in PBS for 10mins, they were processed for antigen retrieval. The slides were firstly boiled for 3 mins in a preheated $0.96 \%$ antigen unmasking solution (Vector Labs), followed by cooling to $50^{\circ} \mathrm{C}$, heating again for 3 mins in the unmasking solution, and finally cooling to room temperature. The slides were then rinsed in PBS for 10mins. Non- specific binding sites on the tissue sections were blocked by incubating in a blocking solution (1\% BSA $+0.1 \%$ Tween-20 in PBS) for $1 \mathrm{hr}$ at room temperature (RT). Primary antibodies were prepared in the same solution and added to sections followed by incubation either at $37^{\circ} \mathrm{C}$ for $1 \mathrm{~h}$ or at $4{ }^{\circ} \mathrm{C}$ overnight. This was followed by atleast two rounds of $10 \mathrm{~min}$ washes in PBS. The sections were then incubated in AlexaFluor- tagged secondary antibodies (1:500, Molecular Probes) also prepared in blocking solution, for not more than $45 \mathrm{mins}$ at RT. The slides were then washed twice in PBS for 10mins per wash, incubated in Hoechst dye 33342 (SIGMA, 1:500) for 5minand mounted in a fluorescent mounting medium (DAKO Cytomation). For immunostainings involving anti- BrdU or anti- IddU antibodies, the DNA in the tissue samples was pretreated by incubating slides in prewarmed $2 \mathrm{~N} \mathrm{HCl}$ at $37^{\circ} \mathrm{C}$ for $30 \mathrm{mins}$, followed by neutralization in borate buffer $\left(0.1 \mathrm{M} \mathrm{Na} \mathrm{N}_{4} \mathrm{~B}_{7}, \mathrm{pH} 8.5\right)$ and two washes in PBS. This was done before blocking non- specific binding sites on the tissue.

Sections obtained using a cryostat were post- fixed in 4\% PFA for 2 mins before washing in PBS, followed immediately by incubation in blocking solution. The rest of the procedure was the same as described above.

\subsubsection{Tyramide- based amplification of signal}

For immunostainings against p-Smad1/5/8 and NICD, the Tyramide Signal Amplification kit TSA $^{\mathrm{TM}}$, Perkin- Elmer, NEL701) was used. The protocol used was similar to that provided in the kit manual. Cryosections were post- fixed in 4\% PFA and washed twice in PBS. The slides were firstly blocked in TNB Buffer $(0.1 \mathrm{M}$ Tris- $\mathrm{HCl}, \mathrm{pH} 7.5+0.15 \mathrm{M}$ $\mathrm{NaCl}+0.5 \%$ blocking reagent supplied in the kit) for $30 \mathrm{~min}$ at $\mathrm{RT}$, followed by the primary antibody (prepared in TNB buffer) at $4{ }^{\circ} \mathrm{C}$ overnight. The sections were then 
washed thrice in TNT buffer $(0.1 \mathrm{M}$ Tris-HCl, $\mathrm{pH} 7.5,0.15 \mathrm{M} \mathrm{NaCl}, 0.05 \%$ Tween20), incubated in biotinylated secondary antibody $(1: 100)$ for $60 \mathrm{~min}$ at RT, washed thrice in TNT buffer, incubated in SA-HRP (Streptavidin-Horse raddish peroxidase) for 30min at RT, and washed thrice in TNT buffer. They were then incubated in Fluorescein- Tyramide (Amplification reagent supplied in the kit and reconstituted in DMSO before use) (1:50) for $10 \mathrm{~min}$ at RT, followed by one $5 \mathrm{~min}$ wash in TNT buffer, incubation in Hoechst dye for $7 \mathrm{~min}$ at RT, and two $5 \mathrm{~min}$ washes in TNT buffer. Finally, the slides were mounted in a fluorescent mounting medium (DAKO Cytomation).

The following is a list of primary antibodies that were used for immunohistochemical analyses-

\begin{tabular}{|c|c|c|c|}
\hline Antigen & Source (species) & Dilution & Manufacturer/Supplier \\
\hline BrdU & Rat & $1: 100$ & Abcam \\
\hline Sip1 & Rabbit & $1: 1000$ & Generated in the lab \\
\hline Brn2 & Goat & $1: 200$ & Santa Cruz \\
\hline Ctip2 & Rat & $1: 250$ & Abcam \\
\hline Ki67 & Rat & $1: 30$ & DakoCytomation \\
\hline Tbr1 & Rabbit & $1: 100$ & Chemicon \\
\hline Foxp1 & Rabbit & $1: 100$ & Abcam \\
\hline Sox 5 & Goat & $1: 100$ & Santa Cruz \\
\hline Satb2 & Rabbit & $1: 1000$ & Generated in the lab \\
\hline Tbr2 & Rabbit & $1: 100$ & Abcam \\
\hline Pax6 & Rabbit & $1: 100$ & Chemicon \\
\hline PH3 & Rabbit & $1: 100$ & Upstate \\
\hline pVim & Mouse & $1: 100$ & MBL \\
\hline $\mathrm{Hu}$ & Mouse & $1: 100$ & Molecular Probes \\
\hline Blbp & Rabbit & $1: 500$ & Chemicon \\
\hline GFAP & Mouse & $1: 500$ & SIGMA \\
\hline Olig2 & Rabbit & $1: 200$ & $\begin{array}{l}\text { Kindly provided by } \\
\text { Dr. John Alberta/ Dr. } \\
\text { Charles Stiles, Dana- } \\
\text { Farber } \quad \text { Cancer }\end{array}$ \\
\hline
\end{tabular}




\begin{tabular}{|l|l|l|l|}
\hline & & & Institute \\
\hline S100ß & Rabbit & $1: 100$ & SWANT \\
\hline Reelin & Mouse & $1: 500$ & Calbiochem \\
\hline p-Smad1/5/8 & Rabbit & $1: 100$ & Cell Signaling \\
\hline NICD & Rabbit & $1: 100$ & Cell Signaling \\
\hline Dcx & Mouse & $1: 100$ & BD \\
\hline Nestin & Mouse & $1: 100$ & Chemicon \\
\hline PCNA & Mouse & $1: 100$ & Novocastra/ Leica \\
\hline
\end{tabular}

\subsection{TUNEL Assay}

We used the Apoptag Fluorescein Direct In situ Apoptosis Detection kit (Chemicon) to detect apoptotic cell death in paraffin embedded sections. We followed the procedure recommended in the product manual.

\subsection{Immunocytochemistry}

Coverslips covered with cultured cells were first fixed in 4\% PFA for $20 \mathrm{~min}$ at RT. They were then washed twice in PBS (without glycine) for 10min per wash, and left overnight in the same solution at $4{ }^{\circ} \mathrm{C}$. The cells were firstly permeabilised in $0.1 \%$ Tween 20 in PBS for $15 \mathrm{~min}$ at RT, followed by blocking of non- specific binding sites with the blocking solution (1\% BSA in $0.1 \%$ Tween20 in PBS) for $1 \mathrm{hr}$ at RT in a humid chamber. Alternatively, droplets of the blocking solution were placed on a parafilm and coverslips were inverted and placed over these droplets; the film was then covered with a plate to avoid evaporation of the solution. The coverslips were then treated with the primary antibodies prepared in blocking solution either for $1 \mathrm{hr}$ at $\mathrm{RT}$ or for $30 \mathrm{~min}$ at $37^{\circ} \mathrm{C}$ followed by $30 \mathrm{~min}$ at RT. They were then washed twice in PBS for $10 \mathrm{~min}$ per wash, and incubated for in 1:500 diluted secondary antibody solution for $1 \mathrm{hr}$ either in a humid chamber or over droplets placed on a parafilm. Finally, excess secondary antibodies were washed off twice in PBS (10min each) and the coverslips were mounted in Prolong Gold Antifade reagent with DAPI (Invitrogen). 


\subsection{Image Acquisition and analysis}

Bright and dark field images were acquired using a compound light microscope (Olympus). Fluorescent stainings were imaged in an inverted Leica Confocal microscope (TCS-SP2 AOBS). Fluorophores were excited using Ar/Kr laser (for A1488 and GFP), $\mathrm{He} / \mathrm{Ne}$ laser (for Al594 and A1647), Blue laser (for A1564 and Cy3), and diode laser (for Hoechst). For slice cultures and for colocalisation studies, images were acquired at a pinhole of 1AU; for others, larger pinholes were used. For colocalisation of GFAP with BrdU/Ki67/Olig2, consecutive images were procured in a z-stack with a step size of 5$8 \mu \mathrm{m}$. These were projected in either two- or three- dimensions, and analysed. Due to the considerable overlap in the excitation and emission spectra of A1488 and Cy3, spectral unmixing was done on the Leica Confocal Software to better separate the signals from the two fluorophores. This facilitated the aforementioned colocalisation analysis and helped avoid misleading results/conclusions due to spectral contamination. Pictures were analysed (merging of overlapping pictures, adjustment of brightness/contrast, quantification, construction of $2 \mathrm{D}$ and $3 \mathrm{D}$ projections etc.) using Leica Confocal software, Adobe Photoshop CS2, CS3 and ImageJ. Statistical analysis was done using Student's t-test (twotailed, unpaired, assuming unequal variance) on Microsoft Excel.

\subsection{Western blotting}

E16.5 wildtype and mutant cortices were dissected out in cooled lysis buffer $(25 \mathrm{mM}$ HEPES pH7.2, 150mM NaCl, 1mM EDTA, 2mM DTT, $0.5 \%$ Triton X-100, cocktail of protease inhibitors), homogenized, and centrifuged at $4{ }^{\circ} \mathrm{C}$ and $1000 \mathrm{~g}$ for $15 \mathrm{~min}$ to remove cell debris. The concentration of proteins in the supernatant was determined by Bradford Assay (Bio-Rad) using known concentrations of BSA to construct the standard curve. The optical densities (ODs) were determined using a spectrophotometer (Ultraspec 1100pro, Amersham, Biosciences) at 595nm. Equal amount of wildtype and mutant protein sample was diluted in 2X Laemmli buffer (125mM Tris-HCl pH6.8; 15\% Glycerol; 3\% SDS; 5\% ß-mercaptoethanol; $0.05 \%$ bromophenol blue), and resolved by electrophoresis on a $10 \%$ SDS-Polyacrylamide Gel (Bio-Rad). The protocol was adapted from 'Current Protocols' and Mini- Protean II Instruction manual (Bio-Rad). A1-2cm-long stacking gel $(0.125 \mathrm{M}$ Tris-HCl pH6.8; 0.1\% SDS; 0.1\% APS; 0.1\% TEMED; 3.2\% acrylamide) and a 5cm-long 
resolving gel (0.375M Tris- $\mathrm{HCl}$ pH8.8; 0.1\% SDS; 0.05\% APS; $0.05 \%$ TEMED; 10\% acrylamide) was used. The samples were separated at $100 \mathrm{~V}$, transferred to a nitrocellulose membrane $\left(0.2 \mu \mathrm{m}\right.$ pore diameter, Millipore) by semi- dry transfer at a rate of $2.5 \mathrm{~mA} / \mathrm{cm}^{2}$ of the membrane for not more than 45min. The Page-Ruler Protein Ladder (Fermentas) was used to estimate the size of separated proteins. The membranes were incubated in blocking solution (5\%Milk in TBS-T (10mM Tris-HCl pH8, 0.9\% NaCl; 0.05\% Tween-20)) for $1 \mathrm{hr}$ at RT followed by the primary antibody at $4^{\circ} \mathrm{C}$ overnight. The following primary antibodies were used- anti- pan-JNK (R\&D Systems, 1:200), anti- NICD (Cell Signaling, 1:100), antiGAPDH (Chemicon, 1:500), anti- pSmad2/3 (SantaCruz, 1:200), anti- Fgfr3 (SantaCruz, 1:200) The membranes were then washed in blocking solution four times, followed by incubation in HRP- conjugated secondary antibody (anti-rabbit-ECL, 1:5000; anti-goatECL, 1:10,000; anti-mouse-ECL, 1:8000) for $1 \mathrm{hr}$ at RT. The above incubation steps were accompanied by mild agitation. The membranes were then washed repeatedly, firstly in blocking solution, then in TBS-T, and finally in TBS buffer. The blots were then treated with a chemiluminescent substrate using standard kits (Amersham ECL WB Detection kit; SuperSignal West Femto Maximum Sensitivity Substrate (PIERCE) detection kit were used for higher sensitivity of detection) and the protocols described by the manufacturers. Finally, the signal was developed onto an X-ray film (X-Omat ${ }^{\mathrm{TM}}$ Blue Films).

\subsection{RNA Isolation, Microarray analysis and cDNA synthesis}

For comparing gene expression between wildytpe and Sip1fl/flEmx ${ }^{\mathrm{Cre}}$ cortices, E13.5 and E14.5 brains were dissected and the total RNA was isolated using the Qiagen miRNeasy minikit. The manufacturer's protocol was followed. The quality and quantity of the isolated RNA samples were determined by agarose- gel electrophoresis. For E13.5 microarray, three wildytpe and three mutant RNA samples were pooled separately, whereas for E14.5, two of each were pooled together. The assay was done at the facility of Dr. Robert Geffers, HCI- Helmholtz centre for infection research, Braunschweig. All RNA samples were stored at $-80^{\circ} \mathrm{C}$. For semi- quantitative PCR (SQ-PCR) at E13.5, three other wildytpe and mutant RNA samples were pooled separately, and alongwith the pools that were analysed by Microarray, were reverse transcribed into cDNA. For SQ-PCR at E14.5, besides the wildtype and mutant samples used for microarray, two wildtype and two mutant RNA samples were synthesized into cDNA. For SQ-PCR at E16.5, three wildtype and three mutant RNA samples were pooled separately and reverse transcribed. 
cDNA synthesis was done using the SMART PCR cDNA Synthesis Kit (BD Biosciences/Clontech) and the protocol was adapted from Evrogen (www.evrogen.com, 'Amplification of cDNA samples using SMART technology'). The first- strand cDNA was synthesized using $2 \mu \mathrm{l}$ RNA sample, Super Script II reverse transcriptase (Clontech), and oligo (dT) primer targeted against the poly-A tail of mRNAs and a template- switch oligo (TS-oligo) that base pairs with and anneals to the deoxycytidine stretch synthesized by the reverse transcriptase at the 3' end of the newly synthesised cDNA strand. The product was diluted and stored at $-20^{\circ} \mathrm{C}$. The first strand cDNA was amplified by a PCR reaction with High fidelity Taq polymerase (USB), an oligo (dT) primer and a primer targeted against the 3' overhanging sequence (complementary to the TS-oligo) that was synthesized by the reverse transcriptase in the previous step. The following amplification program was used$95^{\circ} \mathrm{C}-7^{\prime \prime} ; 62^{\circ} \mathrm{C}-20^{\prime \prime} ; 72^{\circ} \mathrm{C}-2^{\prime} ; 19-22$ cycles. The quality and quantity of cDNA was determined on an agarose gel.

\subsection{Semi- quantitative PCR (SQ-PCR)}

Based on the results of the microarray the following putative targets were chosen for analysis by SQ-PCR:-

E13.5 targets- Peg3, Ramp2, Col3a, Robo1, Aff3, Otub1, sp3, Pdia4, Gt12, Runx1tl, Sfpq, Pfn1, Lgals1, Nap115, PCdh9,

E16.5 targets- Ajuba, Foxo1, Cspg2, Klf9, Dl11, Mth2, Gmfß, Mt1, Notch1, s100a10, sorl1, Gnb1, Fgfr3, Gsta4, Wfdc2, Itga6, Foxo3

E14.5 targets (primers were designed by Marten Kuscher)- Bmpr2, Cbln4, Ebf1, Elov17, Fzd1, Gpr7311, Grb14, Ldb3, Ripk4, Stat4, Stk16, Stx3, Wnt5a, Tuba4.

All cDNA sequences were obtained from NCBI Nucleotide resources. They were aligned with genomic sequence on MapViewer under Mouse Genome Resources in NCBI, for determining the boundary between the last two exons at the 3' end. Alternatively, the ENSEMBL database was also used to determine exon boundaries. In case of those target genes for which only an EST sequence was available on NCBI, this sequence was BLASTed against the mouse genome database and the cDNA that showed maximum identity was chosen to design the primers against. Primers of 20-22 bp with a GC content of $50-60 \%$ were designed manually. They were targeted against the last two exons of the cDNA sequence such that the amplified product would span regions of both exons. This would avoid undesirable amplification of an exon from contaminating genomic DNA. The 
following rules were applied for designing the primers:- 1. No GGG/CCC stretches within the sequence; 2. The last base at the 3' end should be either a $\mathrm{G}$ or a $\mathrm{C} ; 3$. The last two bases at the 5' and 3' end should not be complementary to each other; 4 . The last three bases at the 3 ' end of either primer should not have a reverse and complementary sequence within the primers. Sequences were visualized and aligned on Sequencher v4.2/Gene Codes Corporation). Standard PCR programs with annealing temperatures of $58-62^{\circ} \mathrm{C}$ (calculated using the formula- $\mathrm{T}_{\mathrm{ann}}=2(\mathrm{~A}+\mathrm{T})+4(\mathrm{G}+\mathrm{C})$ ) were used to amplify specified cDNA fragments for 27-29 cycles. The products were then analysed by agarose gel electrophoresis and the relative intensities were compared.

\subsection{In situ Hybridisation}

Expression of the following genes was probed:- Cux2, Oct6, Rorß, Svet1, Er81, Tbr1, mSorla, Sfrp1, Sip1, Nex, Celsr3, Fzd3, Vang12, Lmo4, Stau1, Arvcf, Tmem2, cdc51, cdc25a, Baz2a, Fezl, Coup-TF1.

\subsubsection{Transformation}

Plasmids carrying the desired cDNA fragment were transformed into transformation competent E.coli DH5 $\alpha$ cells. An aliquot of $25-40 \mu 1$ of these cells was thawed, mixed with 1-2 $\mu 1$ plasmid DNA and cooled on ice for $20 \mathrm{~min}$. The cells were permeabilised by treating them to a heat shock. They were first incubated at $42^{\circ} \mathrm{C}$ for $45 \mathrm{sec}$, and then left on ice for 3 min. To the transformed mixture of cells, $500 \mu \mathrm{LB}$ medium (without antibiotics) was added and cultured at $37^{\circ} \mathrm{C}$ for $1 \mathrm{hr}$ with agitation. $100-200 \mu \mathrm{l}$ of this mixture was then plated on LB-antibiotic agarose plates and incubated overnight at $37^{\circ} \mathrm{C}$. Individual colonies were subsequently picked and re-inoculated in 5ml LB (+antibiotic) medium, cultured overnight, and either used for plasmid isolation or mixed with $30 \%$ glycerol to prepare a stock that was stored at $-80^{\circ} \mathrm{C}$.

\subsubsection{Plasmid isolation}

E.coli cells carrying the appropriate plasmid (from a glycerol stock) were inoculated into $5 \mathrm{ml} \mathrm{LB}$ medium containing suitable antibiotics, and cultured overnight at $37^{\circ} \mathrm{C}$ with agitation at $220 \mathrm{rpm}$. The culture was precipitated by centrifugation at $6000 \mathrm{rpm}$ for $5 \mathrm{~min}$. 
Plasmid isolation from the bacterial pellet was done using QIAprep Spin Miniprep kit (Qiagen), according to the specifications in the product manual. For riboprobe synthesis, the isolated plasmid was dissolved in $\mathrm{ddH}_{2} \mathrm{O}$ to a concentration of $100-350 \mathrm{ng} / \mu 1$.

\subsubsection{Plasmid linearization}

Most probe sequences (cloned into plasmids) were flanked by two different types of promoters (usually T3, T7 or Sp6) oriented in such a way that they could be used to synthesise either the antisense or the sense RNA. Therefore, depending on the requirement, the purified plasmids were linearised using a specific restriction enzyme (New England Biolabs) that would cleave the cDNA sequence at the end opposite to the promoter that will be used subsequently for probe synthesis. 1-7 $\mu \mathrm{g}$ of plasmid DNA was digested per reaction. 10units of restriction enzyme was used for linearising $1 \mu \mathrm{g}$ plasmid DNA. Reactions were carried out at $37^{\circ} \mathrm{C}$ for $2 \mathrm{hrs}$ to overnight until the linearisation was completed, which in turn was assayed by gel electrophoresis.

\subsubsection{Purification of linearised plasmid DNA- phenol chloroform extraction}

To precipitate the linearised DNA, 3M sodium acetate $(\mathrm{pH}$ 5.5) was added to the restriction mixture at $1 / 10$ th of the total volume, and vortexed. This was followed by addition of an equal volume of Tris-saturated phenol- chloroform/ isoamylethanol, pH8 (Invitrogen). This mixture was vortexed and then centrifuged at $13,200 \mathrm{rpm}$ for $10 \mathrm{~min}$. The proteins precipitate at the interface as white deposits. The upper phase was then transferred to a new tube and 3 volumes of $100 \% \mathrm{EtOH}+0.5 \mu \mathrm{l}$ coloured co-precipitant Pellet Paint (Novagen) was added to it. The solution was inverted several times and kept at $-20^{\circ} \mathrm{C}$ for 1 hour or at $80^{\circ} \mathrm{C}$ for 30 minutes, to facilitate DNA precipitation. It was then centrifuged at $13,200 \mathrm{rpm}$ for $10 \mathrm{~min}$. The pellet was washed with $70 \%$ ethanol, air dried, and resuspended in $\mathrm{ddH}_{2} \mathrm{O}$ to a concentration of $200-500 \mathrm{ng} / \mu 1$. They were quantified on an agarose gel.

\subsubsection{Riboprobe synthesis (for radioactive in situ hybridization)}

$1 \mu \mathrm{g}$ linearised plasmid was used in $10 \mu 1$ reaction volume to synthesise sense and antisense single stranded RNA probes. The reaction mixture for in vitro transcription consisted of a promoter- specific RNA polymerase (Promega), a mixture of ATP, CTP and GTP 
(Boehringer), and ${ }^{35} \mathrm{~S}$ - labeled UTP (Amersham). The reaction was carried out at $37^{\circ} \mathrm{C}$ for 1-2hrs. Adding Sodium chloride- Tris- EDTA buffer quenched the reaction. Nonpolymerised nucleotides were removed by centrifugation through MicroSpin G-50 gel filtration columns (Amersham). The radioactivity count, and hence, the concentration of the riboprobe was measured in a scintillation counter (Beckman). Based on the count $(\mathrm{cpm} / \mu \mathrm{l})$, we calculated the appropriate amount of hybridization buffer that must be subsequently added to dilute the probe to a concentration of $100,000 \mathrm{cpm} / \mu 1$. The probes were stored at $-20^{\circ} \mathrm{C}$ and used within the next $24 \mathrm{hrs}$.

\subsubsection{Radioactive in situ hybridisation}

Paraffin sections were dewaxed in Histoclear (twice, 10min each) and rehydrated through a series of ethanol solutions (100\% to $30 \%)$ of decreasing strengths. The slides were then rinsed in saline and DEPC- treated PBS, fixed in freshly prepared 4\% PFA for $15 \mathrm{~min}$, washed in PBS twice, treated with proteinase K $(20 \mu \mathrm{g} / \mathrm{ml})$, washed and refixed in PFA, and washed again in PBS. The sections were then treated with a freshly prepared acetylation mix $(0.1 \mathrm{M}$ Triethanolamine; $0.05 \mathrm{M}$ acetic anhydride) in order to acetylate proteins like RNAse. This was followed by rehydration through an ascending series of ethanol solutions ( $30 \%$ to $100 \%)$.

Radiolabeled RNA probes were diluted (to a concentration of $100,000 \mathrm{cpm} / \mu \mathrm{l}$.) in the following hybridization buffer:-

50\% Deionized Formamide (Fulka)

$10 \%$ Hybridization salt stock $(0.2 \%$ polyvinylpyrrolidone; $0.2 \%$ Ficoll; $0.1 \mathrm{M}$

$\mathrm{NaH} 2 \mathrm{PO} 4$; 50mM EDTA pH 6.8; 3M NaCl; 0.1M Tris- $\mathrm{HCl}$ pH8 in DEPC-H2O

10\% 1M DTT (Sigma/Promega)

20\% Dextran sulfate 50\% (Amersham)

$500 \mu \mathrm{g} / \mathrm{ml}$ tRNA (Sigma)

$200 \mu \mathrm{g} / \mathrm{ml} \alpha$ SPthio- ATP (Roche)

The riboprobes were heated at $80^{\circ} \mathrm{C}$ for $2 \mathrm{~min}$ (to denature all secondary structures formed by single stranded RNA) and kept in ice for $5 \mathrm{~min}$. They were then applied onto the paraffin sections, and covered with coverslips that had been siliconised in SurfaSil ${ }^{\mathrm{TM}}$ according to 
the manufacturer's instructions. The sections were incubated overnight at $55^{\circ} \mathrm{C}$ in a chamber humidified with 50\% formamide in 2X SSC buffer.

After hybridization, the coverslips were removed gently and the slides were washed with agitation in preheated $2 \mathrm{X} \mathrm{SSC}\left(55^{\circ} \mathrm{C}, 15 \mathrm{~min}\right), 50 \%$ formamide in $2 \mathrm{X} \mathrm{SSC}\left(75^{\circ} \mathrm{C}, 30 \mathrm{~min}\right)$, $50 \%$ formamide in $2 \mathrm{X} \mathrm{SSC}\left(65^{\circ} \mathrm{C}, 30 \mathrm{~min}\right), 50 \%$ formamide in $2 \mathrm{X} \mathrm{SSC}\left(37^{\circ} \mathrm{C}, 30 \mathrm{~min}-2 \mathrm{~h}\right)$, NTE buffer $\left(0.5 \mathrm{M} \mathrm{NaCl} ; 10 \mathrm{mM}\right.$ Tris-HCl; 5mM EDTA pH8) $\left(37^{\circ} \mathrm{C}, 5+15 \mathrm{~min}\right)$, NTE buffer with RNAse A $(20 \mu \mathrm{g} / \mathrm{ml})$ to wash away unbound RNA probe $\left(37^{\circ} \mathrm{C}, 30+15 \mathrm{~min}\right)$, $50 \%$ formamide in $2 \mathrm{X} \mathrm{SSC}\left(65^{\circ} \mathrm{C}, 30 \mathrm{~min}\right), 2 \mathrm{X} \mathrm{SSC}(15 \mathrm{~min}), 0.1 \mathrm{X} \mathrm{SSC}(15 \mathrm{~min})$, and finally, rehydrated through an ascending ethanol series (30\% to $100 \%)$. The slides were air dried and exposed to Biomax X-ray films (Kodak) overnight.

The radiolabeled slides were embedded in NTB-2 emulsion (Kodak) (pre- warmed at $42^{\circ} \mathrm{C}$ ) briefly, dried, and exposed to the emulsion for 3-7 days at $4^{\circ} \mathrm{C}$, depending on the strength of the signal (as assesses by the signal on the X-ray film). The slides were developed by immersing into chilled 16\% (w/v) Kodak D-19 developer solution for 4min, followed by $1 \%$ acetic acid $(1 \mathrm{~min}), 30 \%$ thiosulfate $(4 \mathrm{~min})$, and $\mathrm{H}_{2} \mathrm{O}(10+10+30 \mathrm{~min})$. They were counterstained in a solution containing $4 \%$ Giemsa stock $(0.85 \%$ Giemsa dye, $50 \%$ glycerol and $50 \%$ methanol) and $0.2 \mathrm{M}$ sodium phosphate, $\mathrm{pH}$. Excess stain was washed away with water. The slides were air- dried and mounted in a hydrophobic mounting medium (Eukitt).

\subsection{Slice Culture}

\subsubsection{Slice culture with Fgf9/NT3 treatment}

E16.5-E18.5 (for Fgf9 experiments) and E13.5-E14.5 (for NT3 experiments) wildtype brains were sectioned into $200-250 \mu \mathrm{m}$ thick slices and cultured according to a protocol modified from that described for Slice overlay assays by Polleux F and Ghosh A in STKE, Jun2002. Isolated brains were first embedded in 3\% agarose (for E16.5 and older brains) or 4\% agarose (for E13.5-E14.5 brains) prepared in cHBSS (1X HBSS, 2.5mM Hepes, 30mM D- glucose, $1 \mathrm{mM} \mathrm{CaCl} 2,1 \mathrm{mM} \mathrm{MgSO}_{4}, 4 \mathrm{mM} \mathrm{NaHCO}$ ) medium. Slices were then cut using a Leica Vibratome and placed on cell culture inserts $(1 \mu \mathrm{m}$ pore size, BD Biosciences) that were in turn placed inside the wells of special 6- well plates (with low 
evaporation lids, notched for use with cell culture inserts, FALCON), filled with 1.5-1.8 ml of Slice culture medium. The inserts were coated overnight with laminin (SIGMA) and poly-L-Lysine (SIGMA). The slice culture medium consisted of complete HBSS, Basal medium Eagle, 20mM D-glucose, $1 \mathrm{mM}$ L-glutamine, Penicillin (100U/ml) / Streptomycin $(0.1 \mathrm{mg} / \mathrm{ml}), \mathrm{N} 2$ supplement $(100 \mu \mathrm{l} / 12.5 \mathrm{ml})$ and $10 \%$ heat inactivated Horse serum. The slices were picked randomly from a pool collected by sectioning 6-8 wildtype brains. Slices were treated with, 1. Different concentrations $(20 \mathrm{ng} / \mathrm{ml}, 50 \mathrm{ng} / \mathrm{ml}$ and $80 \mathrm{ng} / \mathrm{ml})$ of recombinant mouse Fgf9 (Peprotech, 450-30) and cultured for 2-6div, or, 2. Different concentrations (100-600ng/ml) of NT3 (Peprotech, 450-03) and cultured at $37^{\circ} \mathrm{C}$ and $5 \%$ $\mathrm{CO}_{2}$ for 2div. Only one dose was given at approx. $1 \mathrm{hr}$ after plating the slices. After culturing, the slices were fixed in 4\% PFA for 30 mins before immunostaining, which was done according to standard protocols described above. In some cases, $6 \mu \mathrm{g} / \mathrm{ml}$ sterile BrdU was added to the cultures at 1div in order to assess effects of Fgf9 on proliferation in the cortex. For blocking non-specific binding sites, slices were incubated in $0.5 \%$ Triton-X 100 and 2\% BSA in PBS for $1 \mathrm{hr}$ at RT. Primary antibody incubation was done overnight at $4{ }^{\circ} \mathrm{C}$, while AlexaFluor tagged secondary antibodies (Molecular Probes/Invitrogen, 1:500) were incubated at RT for $45 \mathrm{~min}$. All sections were incubated for $10 \mathrm{mins}$ in Hoechst dye (SIGMA, B2261, 1:500) before washing in PBS and mounting in Fluorescent mounting medium (DakoCytomation, s3023).

\subsubsection{Slice culture with implantation of Fgf9/NT3 coated beads}

$100 \mu l$ of agarose beads (Affi-gel Blue gel, Bio-Rad Laboratories) were pipetted into a sterile Eppendorf tube, and allowed to settle. The supernatant was removed and the beads were washed thrice in sterile water by adding $1000 \mu$ of water, inverting the tube gently, spinning briefly on a table- top centrifuge, and decanting off the supernatant after every wash. They were then washed in sterile PBS 4 times in a similar manner. For longer storage, the beads were resuspended in PBS containing Penicillin/Streptomycin $(20 \mathrm{U} / \mathrm{ml})$ and kept at $4^{\circ} \mathrm{C}$. For coating the beads with $\mathrm{Fgf9}, 20 \mu \mathrm{l}$ bead solution was mixed with $10 \mu 1$ of $200 \mathrm{ng} / \mu \mathrm{Fgf9}$ solution and incubated overnight at $4^{\circ} \mathrm{C}$. For coating with NT3, the beads were soaked in $20 \mu \mathrm{l}$ of $100 \mu \mathrm{g} / \mathrm{ml}$ NT3 stock solution and kept at $4^{\circ} \mathrm{C}$ overnight. As control, we coated the beads with BSA; $20 \mu$ l bead solution was mixed with $20 \mu$ of $1 \mathrm{mg} / \mathrm{ml}$ BSA solution. 
Capillaries of appropriate nozzle size were prepared in a temperature- controlled pipette puller (PIP5, HEKA) using a two step- method. In the first step, the capillary was melted and stretched, while in the second step, it was melted and the lower half was allowed to stretch and fall freely.

For experiments with Fgf9, E16.5-E17.5 wildtype slices (200 $\mu$ m thick) were sectioned on a Leica vibratome and placed on cell culture inserts inside a 6-well plate, as described above. For experiments with NT3, E13.5 wildtype slices $(300 \mu \mathrm{m}$ thick) were prepared. Before placing the beads on the slices, they were first washed in a large volume of sterile PBS, at least twice, with brief centrifugation between each washing step in order to collect as many beads as possible. Finally, they were washed and then resuspended in $100 \mu 1$ SCM. This solution was then placed on a sterile petriplate as a droplet. Using a mouth pipette, beads of desired size were picked from this droplet by applying slight negative pressure, while observing them under a compound microscope. They were then placed either over the cortical plate or the VZ/SVZ by gently touching the surface of the tissue with the bead; it was not necessary to apply positive pressure to release the beads. Important here is to pick up beads of the right size; too small beads would pass thought he nozzle and very large ones would be difficult to hold at the tip of the capillary. The beads therefore should be of the appropriate size so that they can be held on the tip of the capillary with a slight negative pressure, and which can be easily placed on a slice without having to blow through the pipette.

The cultured slices were fixed after 2div, and immunostained as described before.

\subsection{Slice overlay assay}

This assay was performed according to the protocol described by Polleux F and Ghosh A in STKE, Jun2002. E16.5-E18.5 wildtype brain slices were prepared and plated on cellculture inserts as described before and in the aforementioned publication. E12.5 cortices were dissected from wildtype (GFP-) or transgenic GFP expressing brains in cHBSS. They were then incubated in a solution of papain $(400 \mathrm{U} / 20 \mathrm{ml})$, cysteine $(6.4 \mathrm{mg} / 20 \mathrm{ml})$ and Dissociation medium (DM $\left(98 \mathrm{mM} \mathrm{Na}_{2} \mathrm{SO}_{4}, 30 \mathrm{mM} \mathrm{K}_{2} \mathrm{SO}_{4}, 5.8 \mathrm{mM} \mathrm{MgCl}_{2}, 0.25 \mathrm{mM} \mathrm{CaCl}_{2}\right.$, $1 \mathrm{mM}$ Hepes, $20 \mathrm{mM}$ glucose, $0.001 \%$ Phenol red, $0.125 \mathrm{mN} \mathrm{NaOH})$ ) for $5-10 \mathrm{~min}$ twice, followed by sequential washing in heavy inhibitory solution (60mg BSA + 60mg Trypsin inhibitor in $6 \mathrm{ml} \mathrm{DM}$ ) and light inhibitory solution (1:10 diluted heavy inhibitory solution). Finally, the cells were washed in serum free medium $(100 \mathrm{ml}$ Basal medium Eagle $+1 \mathrm{ml}$ 
$\mathrm{N} 2$ supplement $+0.5 \mathrm{ml} 200 \mathrm{mM}$ L-glutamine $+1 \mathrm{ml}$ Penicillin $(10,000 \mathrm{U} / \mathrm{ml}) /$ Streptomycin $(10 \mathrm{mg} / \mathrm{ml}))$, and resuspended in a fresh aliquot of the same. The number of viable cells was determined by firstly treating an aliquot with Trypan Blue, and then counting the cells using a hemocytometer. Approximately $10^{5}$ to $5 \times 10^{5}$ cells were plated on top of the slices in each well. The cultures were fixed and immunostained after 5-6div, according to the protocol described above.

\subsection{Explant culture (with NT3 treatment)}

E12.5 wildtype and/or mutant brains were dissected out, the basal ganglia were removed, and only the cortices were plated onto laminin and poly-L-Lysine coated cell culture inserts using a wide- mouthed pipette. In one set of experiments, only wildtype explants were cultured in different concentrations of NT3 in the SCM (100-600 ng/ml). In the second set of experiments, wildtype and mutant explants from littermates were cultured in the presence of $10 \mu \mathrm{g} / \mathrm{ml}$ anti-human NT3 function blocking antibody (Promega) or chicken IgY (control). The explants were cultured in SCM for 2-3div before fixing in 4\% PFA for $30 \mathrm{~min}$. The same immunostaining procedure that was used for cultured slices was used here as well.

\subsection{Dissociated cortical cell culture}

Dissociated cells from E12.5 and E13.5 cortices were prepared as described above, and plated on coverslips at a concentration of $0.5 \times 10^{5}$ to $2 \times 10^{5}$ cells per well of a 12- well plate. The latter were coated overnight with poly-L-Lysine and laminin. In the first set of experiments, different dilutions of NT3 $(50,100,200,400,600 \mathrm{ng} / \mathrm{ml})$ were prepared in SCM and added to the cells after they adhered to the coverslips (approx. $1 \mathrm{hr}$ after plating). The cells were then cultured for 2-3 div, fixed in 4\% PFA for 20 min and immunostained for specific markers (Satb2, Ctip2, Sox5, Foxp2).

In the second set of experiments, E12.5 and E13.5 cortical cells were isolated from wildtype and Sip1fl/flNex ${ }^{\text {Cre }}$ brains. $5 \times 10^{4}$ cells per well were plated on coated coverslips of a 24- well plate. 2-3 hrs after plating cells, anti-human NT3 function blocking antibody or chicken IgY (control) was added to the well at a concentration of $20 \mu \mathrm{g} / \mathrm{ml}$. The cultures were fixed after 36- $48 \mathrm{hrs}$, and immunostained for Satb2 and Ctip2. The above protocol was designed based on the experiments reported in (Barnabe-Heider and Miller, 2003). 


\section{RESULTS}

\subsection{Sip1 is expressed in postmitotic cells of the neocortex}

We performed a series of immunostainings against Sip1 in order to characterise its pattern of expression through embryonic development and at early postnatal stages. From these experiments, we could detect expression of Sip1 only in postmitotic cells of the neocortex (Fig2a). Interestingly, when we performed in situ hybridisation to detect Sip1 transcripts, very weak expression of the latter was seen in the germinal zones as well (data not shown). Sip1 expression is seen as early as E12.5 when the first neurons of the CP are born and lasts until early postnatal stages (until P8); at the latter stages, very few cells continue to express high levels of Sip1 while most cortical neurons downregulate its expression (Fig2h-1). At P4, Sip1 is strongly expressed in the upper layers and in layer 6, but only very few cells in layer 5 show strong Sip1 signal. At certain time points, we have also detected weak Sip1 expression in the VZ/SVZ, where some cells are GFAP+ astrocytes. At E17.5, expression of Sip1 in the cingulate cortex gets restricted to layers 5 and 6 , while none is detected in upper layer neurons (Fig2g). However, in the dorsal/lateral differentiating field of the neocortex, Sip1 continues to be expressed in all cells. We next decided to characterise the Sip1 expressing cells of the neocortex. We found that it is expressed in different cell types. At E15.5, all upper layer neurons expressing Brn2 and all deep layer neurons that express Ctip1 also stain for Sip1 (Fig2d,e). At E17.5, Sip1+ cells coexpress either upper layer marker Brn2 or layer 5 marker Ctip2. However, not all Brn2 or Ctip2 expressing cells coexpress Sip1- the two populations seem to subdivide into Sip1+ and Sip1- cells. (Fig2b,c). Besides neurons, we also detected Sip1 expression in some Reelinsecreting cells of the marginal zone and the cortical plate at early postnatal stages (Fig11d,e), and in few GFAP+ astrocytes close to the pial surface. Furthermore, expression of Sip1 protein seems to be exclusive to postmitotic cells of the cortex since none of the proliferating cells (labeled with an antibody against Ki67) express Sip1 at any stage of development (Fig2f). Ki67 is a protein that can be detected in the nucleus during all active phases of the cell cycle, but is absent in quiescent cells; it is therefore used as a marker of cell proliferation (Gerdes et al., 1983; Scholzen and Gerdes, 2000). 

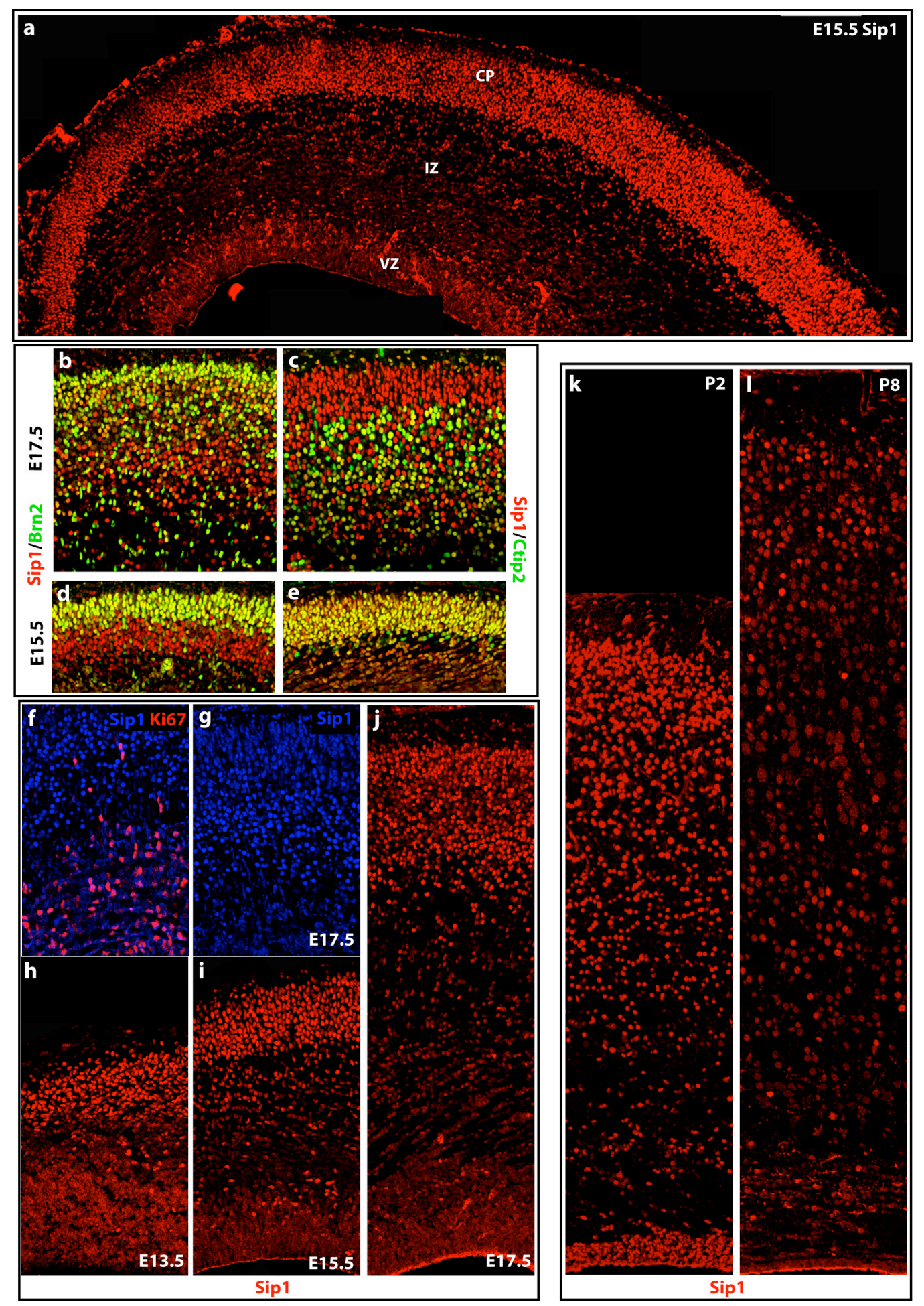

Fig2. Sip1 is expressed strongly and predominantly in postmitotic cells of the neocortex. Immunostaining for Sip1 shows protein expression in the cortical plate at E15.5 (a), although a cohort of Sip1+ interneurons migrating tangentially from the ganglionic eminences can also be seen in the SVZ/IZ of the neocortex. Sip1 is expressed by both upper (Brn2+) as well as deep (Ctip2+) layer neuronal types. However, while at E15.5, all Brn2+ and Ctip2+ cells express Sip1 (b,c), by E17.5 the former populations segregate into Sip1+ and Sip1- cell types (d,e). Sip1 is not expressed in dividing cells (shown here is colabelling with proliferation marker Ki67 at E17.5) (f). At E17.5, Sip1 expression gets restricted to layer 5-6 
neurons only in the cingulate cortex (g). Sipl continues to be expressed strongly in the cortical plate throughout embryonic development until early postnatal stages (h-1).

\subsection{Conditional ablation of Sip1 in cortical progenitors and/or postmitotic neurons}

Due to the embryonic lethality of Sip1 total knockouts (Van de Putte et al., 2003), it was not possible to use them to study Sip1 function in corticogenesis, which begins at E11.5 (2 days after the embryos die) in mice. Hence, we decided to generate and analyse conditional knockouts of Sip1. These mutants carry 2 copies of floxed Sip1 allele (exon7) (Higashi et al., 2002) alongwith a Cre recombinase coding sequence under the control of a tissue/stage - specific promoter. Important functional domains of sip1 protein, including the Smadbinding domain, the homeodomain and the fourth $\mathrm{N}$ - terminal zinc finger, are encoded by exon7 of Sip1 gene (Maruhashi et al., 2005). For the purpose of our studies, we used primarily two Cre-lines, 1. Cre under the Emx1 promoter (Gorski et al., 2002). This promoter is known to be active as early as E9.5 specifically in the cortex (neocortex and hippocampus); hence, Sip1fl/fl-Emx ${ }^{\mathrm{Cre}}$ mutants delete Sip1 in all cortical progenitors and postmitotic cells of the cortex. 2. Cre under the Nex promoter (Goebbels et al., 2006). In the neocortex, this promoter is active only in postmitotic neurons, but not in the germinal zones, interneurons, astrocytes and oligodendrocytes (Goebbels et al., 2006).

To begin with, we confirmed ablation of Sip1 in these mutants by immunostaining. Deletion of Sip1 can be observed already at E13.5 in both Sip1fl/fl-Nex ${ }^{\mathrm{Cre}}$ as well as Sip1fl/fl-Emx ${ }^{\text {Cre }}$ (Fig3a-d). A small fraction of cortical cells seem to escape recombination and continue expressing Sip1. Interestingly, Sip1 is expressed in the cells of the basal ganglia as well. Since neither of the Cre lines are active in the basal ganglia, the cohort of Sip1+ cells seen in the conditional knockouts in the SVZ/IZ and close to the MZ are possibly interneurons born in the ganglionic eminences that migrate tangentially into the cortex. A few Sip1+ cells are also detected in the MZ. 


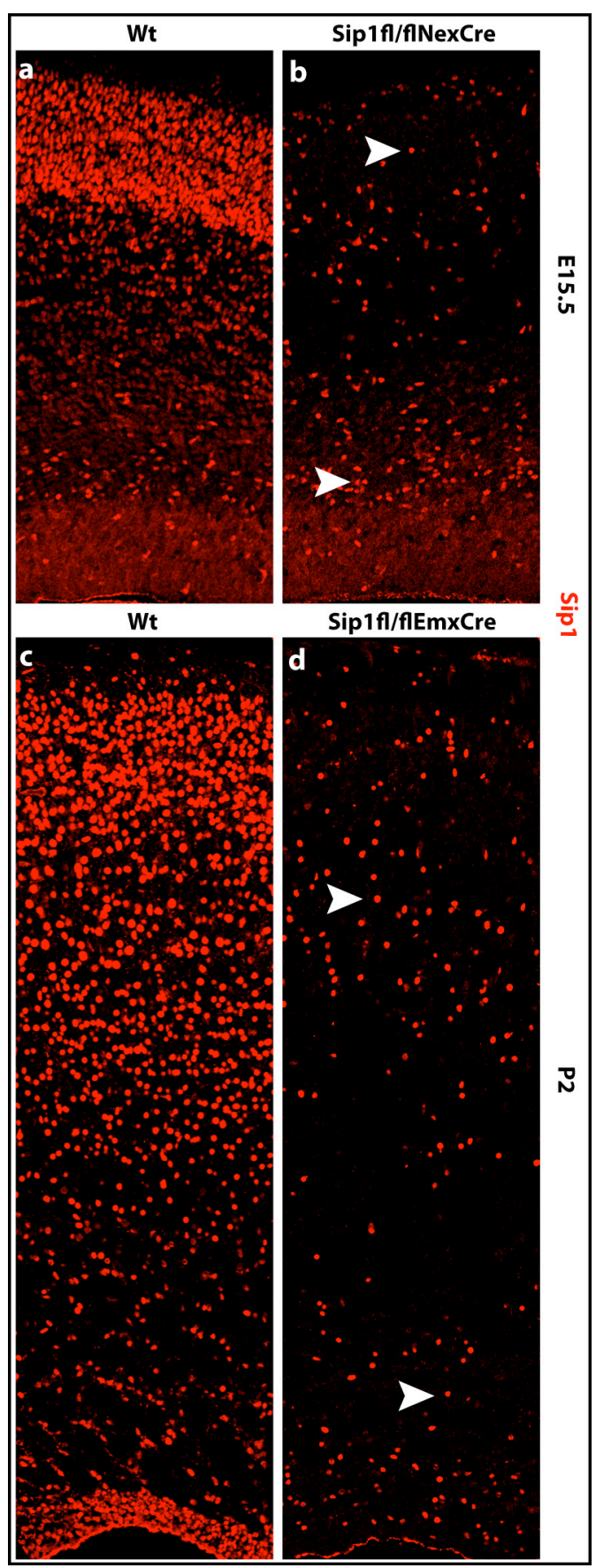

Fig3. Conditional knockouts of Sip1 ablate its expression specifically in the cortex. Two conditional knockouts were generated and studied- floxed Sip1 allele in combination with, 1. Cre under the Nex promoter (designated as Sip1fl/flNexCre), and 2. Cre under the Emx1 promoter (designated as Sip1fl/flEmxCre). Shown here is the lack of Sip1 expression in the two mutants in comparison to wildtype control cortices at E15.5 (a,b) and P2 (c,d). A small percentage of cells continue to express Sip1 (arrowheads); these could either be cells that escaped recombination randomly, or they could be tangentially migrating interneurons from the ganglionic eminences where Sip1 is strongly expressed and is not deleted in the above mutants. 


\subsection{Morphology of Sip1- deficient cortices}

\subsubsection{Conditional Sip1 mutants have a thinner cortex with disorganised stratification of neurons, and absence of corpus callosum and anterior commissure}

As reported earlier, conditional Sip1 mutants lack the corpus callosum and the anterior commissure (Doctoral Thesis, Miquelajauregui A, Mar 2006, University of Goettingen, unpublished data). However, they do contain the Probst bundle on either hemisphere. In order to study the gross morphology of the mutant cortex, we stained all nuclei with the DNA- binding dye Hoechst in postnatal brain sections. The thickness of the cortex is reduced, and the different neuronal layers that are arranged compactly in the wildtype, are dispersed and seem to be loosely packed in both Sip1 conditional knockouts (Fig11a-c). There also seem to be more cells close to the ventricle and in the intermediate zone (IZ). This phenotype was found consistently in all postnatal mutant brains that we studied. Hypoplasia (incomplete development) of the cortex could be due to several reasons including reduced proliferation, increased apoptosis, shortened period of neurogenesis, etc. On the other hand, as a cause of dispersed cortical layers, we suspected either defects in migration of neurons born in the cortex, or alterations in cell fate specification and the dynamics of production of different neuronal types. We performed a series of experiments to address all of these possibilities.

\subsubsection{Absence of Sip1 in the cortex leads to a reduction in deep layer neurons accompanied by an expansion of upper layer cell types}

Our analysis of the neocortical phenotype in Sip1 conditional mutants began with a study of the number, position and distribution of different cell types that constitute the six layers of the cortex. To achieve this, we immunostained wildtype and mutant cortices for a host of layer-specific molecular markers. We used Ctip2 for labeling predominantly layer 5, though it also labels a subpopulation of layer 6 neurons, Foxp 1 and Sox 5 for layer5/6, and Tbr1 for layer 6 (Arlotta et al., 2005; Bulfone et al., 1995; Hevner et al., 2001; Lai et al., 2008). Our results show that the Sip1 mutant cortex consists of fewer deep layer neurons than the wildtype (Fig 4a-f, Fig5a-d). In both mutants, we observed a decrease in the number of layer 5 and 6 neurons as early as E15.5 until early postnatal stages (Fig4k,l). In case of Sip1fl/fl-Nex ${ }^{\mathrm{Cre}}$ the reduction in layer 6 neurons was more profound and could be 
again detected as early as E15.5, while the reduction in layer 5 neurons was less obvious at this stage. Interestingly, by P8 (when expression of Sip1 in the cortex gets highly diminished (Fig21)) no differences in the number of Ctip2+ cells could be seen; however, layer 6 neurons were still strongly reduced. We found Foxp1 and Sox 5 to be expressed predominantly in layer 5 and to a lesser extent in layer 6 at E17.5 (Fig5a,c). In Sip1fl/fl$\mathrm{Nex}^{\mathrm{Cre}}$ cortices, not only did we observe fewer cells of each type, but also more cells expressing both the transcription factors (Fig5b,d,e-f). While in the wildtype, a considerable number of Foxp1+Sox5- cells can be seen in layer3 (Fig5e), in Sip1fl/fl$\mathrm{Nex}^{\mathrm{Cre}}$, such cells are hardly detected (Fig5f). Next, we looked at upper layer neuronal populations by immunostaining for markers Satb2 and Brn2 (Britanova et al., 2005; Sugitani et al., 2002). At prenatal stages of development, there is a definite increase in the thickness of layers 2-4 (Fig4g-j). At early postnatal stages, however, we did not observe any obvious expansion. It is known that at E15.5 a significant number of cells in the cortical plate express both Satb2 as well as Ctip2. These cells are believed to represent a transitory stage before differentiating into Satb2+ upper layer neurons (Britanova et al., 2008). Interestingly, we observed an increase in the number of Satb2+Ctip2+ cells in mutant cortices at E15.5 (Fig5i,j), probably as a consequence of premature differentiation of cortical progenitors towards an upper layer lineage. While in the wildytpe, several

Satb2+Ctip2- cells could be detected (Fig5i), they were hardly seen in Sip1fl/fl-Nex ${ }^{\mathrm{Cre}}$ (Fig5j). We observed a similar mixed identity of deep layer neurons at E16.5; while in wildtype cortices, a significant number of Ctip2+Tbr1- layer 5 neurons could be seen, in Sip1fl/fl-Emx ${ }^{\text {Cre }}$, very few of these cells exist at this stage and most Ctip2+ cells coexpress Tbr1 (Fig5g,h). Interestingly, in comparison to the wildtype, many more Tbr1+Ctip2- cells can be seen in the mutant cortex at this stage (Fig5g,h).

\subsubsection{Ablation of Sip1 has no effect on early proliferation and cell cycle kinetics of cortical progenitors}

During early corticogenesis (E12.5-E15.5), we did not observe any consistent changes in the level of proliferation both at the ventricular zone (VZ) as well as at the subventricular zone (SVZ) in Sip1 mutants. We labeled all M-phase cells using an antibody against phospho-Histone3 (PH3) (Fig6a-i) and M-phase radial glial cells using an antibody against phospho-Vimentin (pVim) (Fig6a-c) (Hendzel et al., 1997; Kamei et al., 1998). At E12.5- 


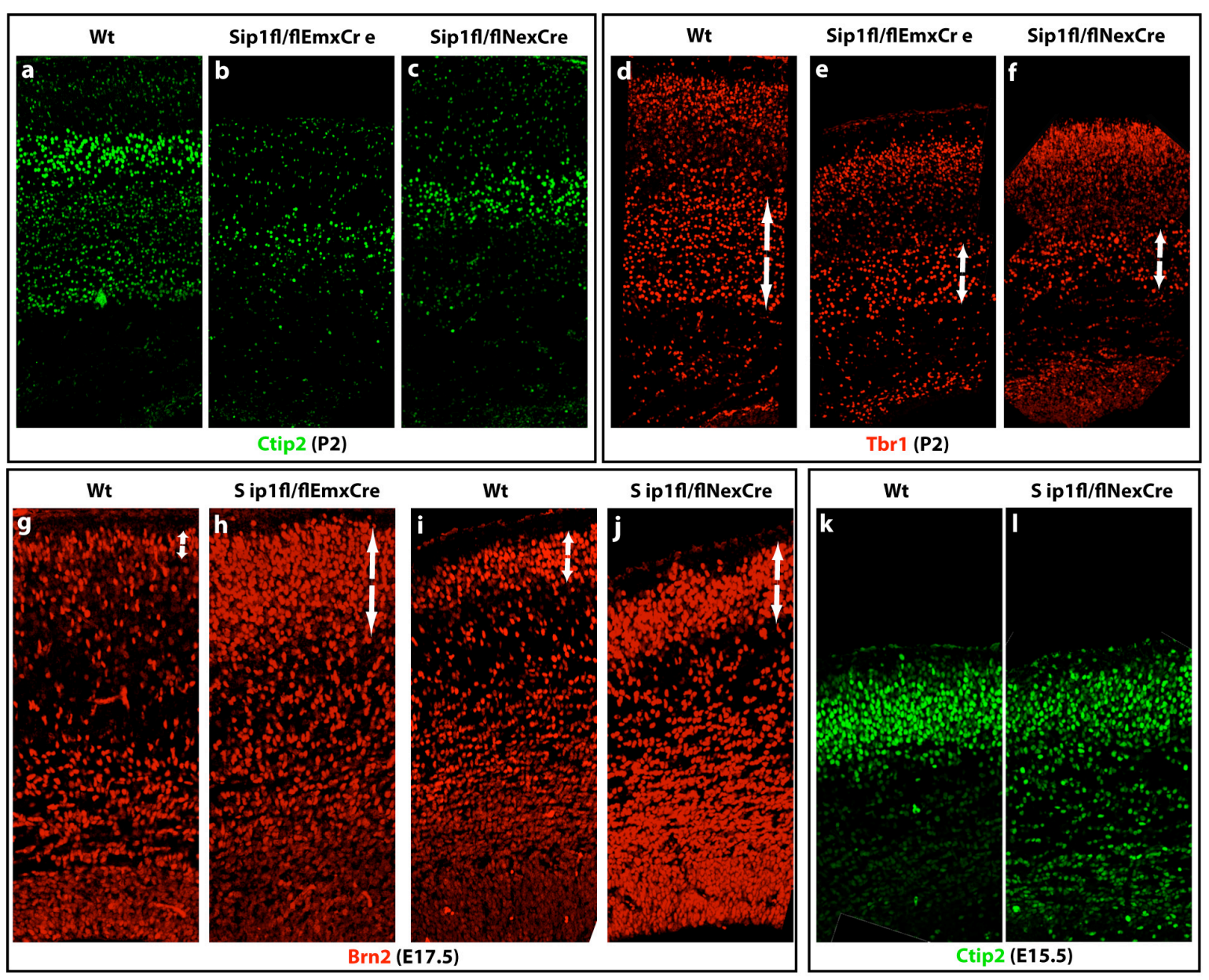

Fig4. Deletion of Sip1 leads to reduced number of deep layer neurons concomitant with increased numbers of upper layer neurons at prenatal stages of development. Immunostaining for different layer 5/6 specific molecular markers (Ctip2 (a-c) for layer5 and Tbr1 (d-f, arrows) for layer 6) at P2 shows that the Sip1 mutant cortex consists of fewer deep layer neurons than the Wt. In case of Sip1fl/fl-Nex ${ }^{\text {Cre }}$, we observed a decrease in the number of Ctip2+ neurons as early as E15.5 $(\mathrm{k}, 1)$. Immunostaining for upper layer neuronal markers (Satb2 and Brn2 $(\mathrm{g}-\mathrm{j}))$ indicates an increase in their numbers at prenatal stages of development. Shown here is expression of Brn2 in Sip1fl/fl-Emx ${ }^{\mathrm{Cre}}$ at E17.5 (g,h) and in Sip1fl/fl-Nex ${ }^{\mathrm{Cre}}$ E15.5 (I,j).

E15.5, while most $\mathrm{PH} 3+$ and $\mathrm{pVim}+$ cells were distributed around the lumen, another cohort of M-phase cells was found localised to the basal VZ/ presumptive SVZ (Fig6a-i). Three sub-populations of M-phase cells were seen- PH3+pVim-, PH3-pVim+, and, $\mathrm{PH} 3+\mathrm{pVim}+$. Neither of these were altered in Sip1 mutants. We then adopted an alternative approach to study possible alterations in proliferation in the cortex by labeling a subgroup of dividing cells (those that are at the S-phase of cell cycle) at E15.5 with a 1 and $1 / 2 \mathrm{hr}$ BrdU pulse. BrdU is a thymidine analog that integrates into replicating DNA at the 
S-phase of cell cycle (Hardonk and Harms, 1990). This was followed by immunostaining for the BrdU label (Fig4j-1); from this analysis, we did not detect any obvious differences in the extent of proliferation.
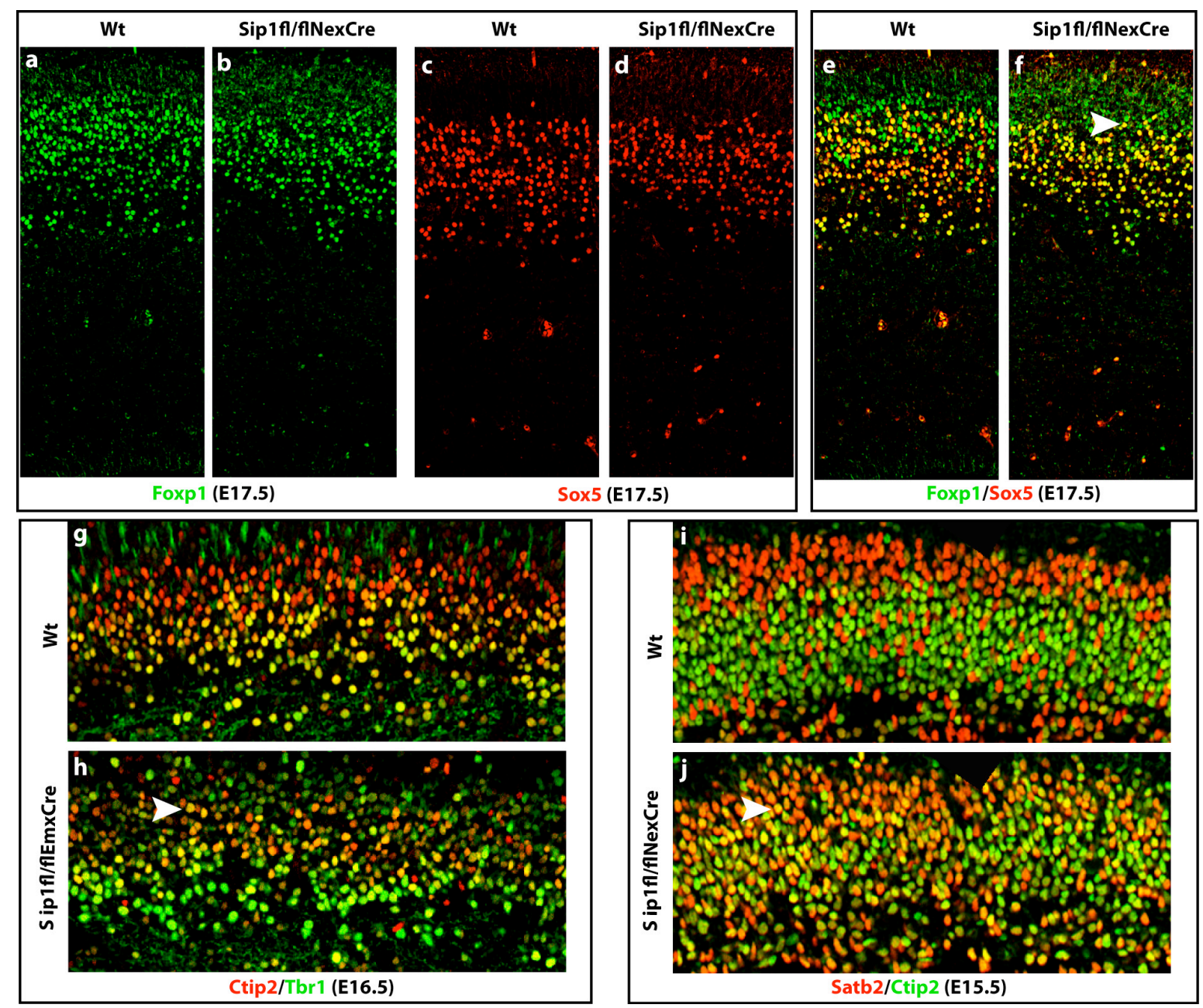

Fig5. Reduction of cortical layers $5 / 6$ in Sip1 conditional mutants is accompanied by delayed maturation and/or mixed identity of some deep layer neurons. Expression of layer5/6 markers Foxp1 $(a, b)$ and $\operatorname{Sox} 5(c, d)$ at E17.5 is reduced in $\operatorname{Sip} 1 \mathrm{fl} / \mathrm{fl}-\mathrm{Nex}^{\mathrm{Cre}}(\mathrm{b}, \mathrm{d})$ in comparison to the wildytpe $(\mathrm{a}, \mathrm{c})$. In both mutants, cells coexpressing two different layer- specific markers (e-j, arrowheads) were more in number than those expressing exclusively one marker, reflecting either mixed neuronal identity or delayed maturation. Shown here is costaining for Foxp1 and Sox 5 at E17.5 in Sip1fl/fl- $\mathrm{Nex}^{\mathrm{Cre}}(\mathrm{e}, \mathrm{f})$, Ctip2 and Tbr1 at E16.5 in Sip1fl/fl-Emx ${ }^{\text {Cre }}(\mathrm{g}, \mathrm{h})$, and Satb2 and Ctip2 at E15.5 in Sip1fl/fl-Nex ${ }^{\mathrm{Cre}}(\mathrm{I}, \mathrm{j})$.

Next, we decided to study specific progenitor types of the VZ/SVZ. In order to achieve this, we immunolabeled wildtype and mutant cortices with antibodies against Pax6 (expressed by most cells of the VZ) (Fig6o,p), Nestin and Blbp (expressed by radial glial cells), and Tbr2 (expressed by cells of the SVZ) (Fig6m,n) (Englund et al., 2005; Feng et 
al., 1994; Gotz et al., 1998; Misson et al., 1988). We found no differences in the expression of Pax6. Hence, we concluded that ablation of Sip1 either in progenitors or postmitotic cells or both, does not affect proliferation of $\mathrm{VZ}$ progenitors during the peak period of neurogenesis. However, when we examined the expression of Tbr2 closely, it seemed to us that there were many more Tbr2+ cells basal to the SVZ (in the IZ) in the wildtype in comparison to Sip1fl/fl-Emx ${ }^{\text {Cre }}$ (Fig6m,n). This observation has been discussed later. We also used anti-Hu antibody to mark postmitotic neurons, and found no difference in the thickness of $\mathrm{Hu}$ expressing cortical plate during early development (Fig6d-f).

In order to study possible alterations in the mitotic cycle of cortical progenitors, we studied two crucial parameters- Mitotic exit, and interkinetic nuclear migration. In order to determine the rate of mitotic exit, we calculated the proportion of dividing cells that quit the cell cycle and became postmitotic cells within $24 \mathrm{hrs}$. For this, BrdU was injected into pregnant females at E12.5 and the embryos were dissected out and fixed at E13.5; the cortices were then stained for BrdU (Fig6q,u) and the proliferation marker Ki67 (Fig6r,v). The fraction of cells that stain for BrdU but not Ki67 amongst the population of BrdU+ cells essentially reflects the rate of mitotic exit (Fig6s,w) (Chenn and Walsh, 2002). From E12.5 to E13.5, we found that about $14 \%$ cells exit the cell cycle. This percentage was not different in Sip1fl/fl-Emx ${ }^{\mathrm{Cre}}$ mutant cortices. (based on preliminary quantification).

Once a cohort of S-phase cells is labeled through BrdU injection, based on the position of the tagged cells after definite time intervals, it is possible to speculate on whether the movement of nuclei at the VZ towards and away from the ventricle is affected or not. In wildtype embryos, one hour after BrdU labeling, the nuclei are located away from the apical surface of the VZ, while 6 hours later they return back to the ventricle. We performed this experiment at E15.5 and found no significant differences between wildtype and Sip1fl/fl-Emx ${ }^{\text {Cre }}$ (data not shown) (Gotz et al., 1998). Altogether, it seems that Sip1 has no influence on cell cycle regulation in the developing neocortex.

\subsubsection{Apoptosis is not enhanced during embryonic development in the absence of Sip1}

Since we observed a reduction in deep layer neuronal types already at E15.5, we speculated that in case increased apoptosis was responsible for this, we should be able to detect it at this stage or perhaps even earlier through TUNEL assay. At E14.5 and E15.5 

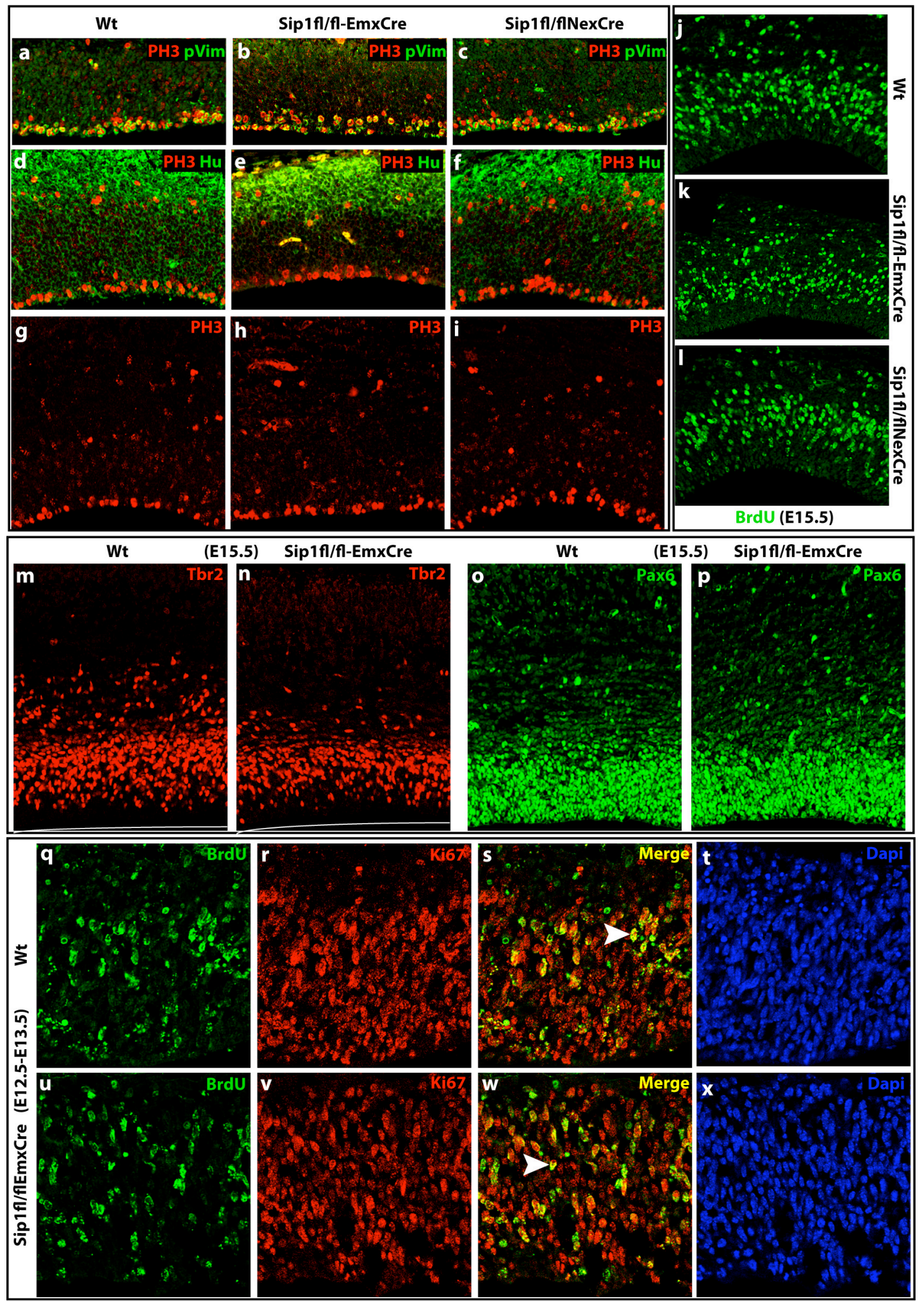

Fig6. Sip1 does not regulate proliferation and cell cycle kinetics during early corticogenesis. At E12.5 (a-c), M- phase cells were detected by labeling for PH3 and pVimentin; at E13.5 (d-f) besides PH3, young postmitotic neurons were additionally labeled for Hu; at E15.5 (g-i), PH3 staining was used to detect both apically as well as basally dividing cells. No differences were seen in the level of proliferation between wt 
and either Sip1 mutant, as shown here by a 1 and $1 / 2$ BrdU pulse at E15.5 (j-1). Expression of Pax6 in the VZ $(\mathrm{o}, \mathrm{p})$ at E15.5 is unaltered, while that of Tbr2 in the $\operatorname{SVZ}(\mathrm{m}, \mathrm{n})$ seems reduced, especially in the IZ/basal SVZ. The rate at which cells exit the cell cycle is determined by labeling dividing cells with BrdU (q,u) 24 hrs prior to analyzing them for expression of Ki67 (r,v) expressed at all stages of cell cycle. The fraction of BrdU+ cells that no longer express $\operatorname{Ki67}(\mathrm{s}, \mathrm{w})$ represent the rate of mitotic exit. Based on preliminary quantification, no differences could be detected in this fraction between wt and mutants. Arrowheads indicate cells expressing both BrdU and Ki67.

we detected very few apoptotic nuclei in the VZ/SVZ in both wildtype and Sip1fl/fl$\mathrm{Emx}{ }^{\mathrm{Cre}}$, but none in the cortical plate. No obvious differences in the number of TUNEL+ nuclei could be found.

\subsubsection{Sip1 controls proliferation and specification of astrocytic precursors during later stages of corticogenesis}

Although proliferation during the primarily neurogenic period (E11.5-E16.5) of corticogenesis did not seem affected by the absence of Sip1, our experiments at later stages showed otherwise. In order to label a subpopulation of dividing cells (those in the Sphase), we labeled E17.5 embryos with a 1 and 1/2 hr BrdU pulse injected intraperitoneally into pregnant females (Fig7a,b). We also immunostained fixed samples for proliferation markers such as Ki67, PCNA, Pax6 ( to label VZ progenitors) Tbr2 (to label SVZ progenitors), Blbp (to label astrocytic precursors), and PH3 (to label M-phase cells). We detected an enhanced and abnormal proliferation in the cortex, especially in the region of the cingulate cortex. As has been quantified here, not only did we find more dividing cells, they also seem to be dispersed largely over the SVZ/IZ/CP instead of being localised mostly to the VZ/SVZ as in wildtype brains (Fig7a,b,o). We divided one radial unit into five equal sized bins and counted the number of Ki67+ cells within each bin. The immunostaining against Tbr2 shows clearly how most VZ progenitors are either absent or displaced in the mutant cingulate cortex $($ Fig $7 \mathrm{~m}, \mathrm{n})$. Interestingly, almost all of these abventricularly dividing cells express the radial glial marker Blbp (Fig7c-h). It has been claimed that expression of Blbp is characteristic to differentiating astrocytic precursor cells (Hartfuss et al., 2001; Nieto et al., 2001; Rousselot et al., 1997). Also, most though not all of these cells express Pax6; cells expressing Pax6 are also dispersed through the cortex (Fig7i,j). Moreover, there seem to be more cells expressing Tbr2 in the SVZ of the cingulate cortex; interestingly, in both wildtype and mutant cortices, almost the same 


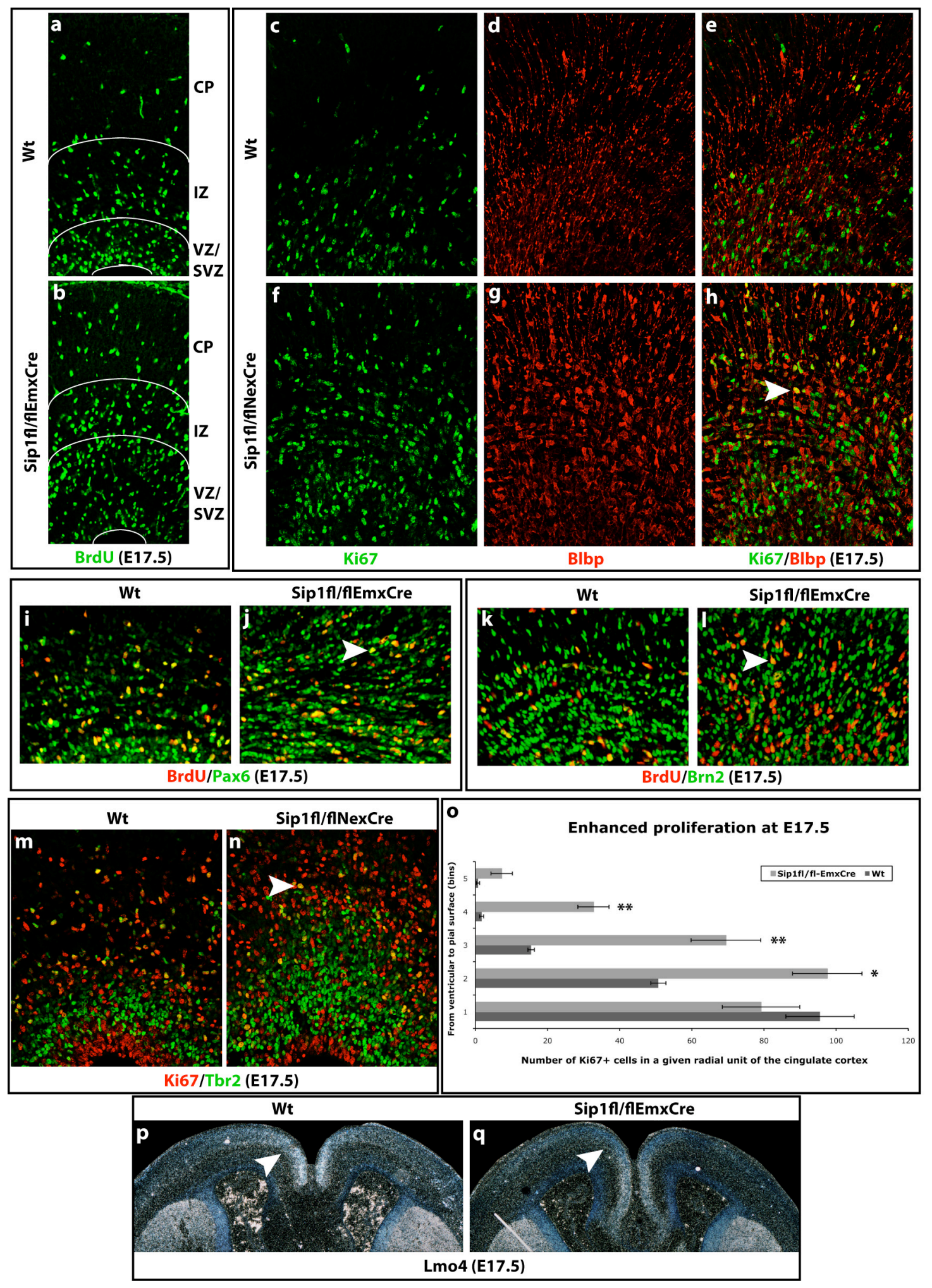

Fig7. Conditional ablation of Sip1 induces increased proliferation of neocortical astrocytic precursors at E17.5. A 1 and $1 / 2 \mathrm{hr}$ BrdU pulse to label one fraction of dividing cells at E17.5 shows an increased number of progenitors, many of which are dispersed away from the VZ in the mutant cingulate cortex (a,b, quantified in o). The entire radial unit from the ventricle to the pial surface in the region of the cingulate 
cortex was divided into five equal- sized bins numbered 1 to 5 (shown in the y-axis); within this area, Ki67+ cells were counted (o). Student's t-test was performed using $3 \mathrm{Wt}$ and 3 mutant brains; bin2 * $\mathrm{p}=0.0098$, bin3 $* * \mathrm{p}=0.005$, bin $4 * \mathrm{*}=0.0019$. Most ectopically dividing Ki67+ cells express the astrocytic radial glial marker Blbp in mutants (f-h, arrowhead), while very little coexpression is detected in wildtype cortices at E17.5 (ce). Most abventricularly dividing cells show strong Pax6 expression, while only some contain high levels Brn2; most cells either stain weakly or not at all for Brn2. Shown here is colocalisation of Pax6 and Brn2 with BrdU pulsed for 1 and $1 / 2 \mathrm{hr}$ in $\mathrm{Wt}(\mathrm{i}, \mathrm{k})$ and $\operatorname{Sip} 1 \mathrm{fl} / \mathrm{fl}-\mathrm{Emx}{ }^{\mathrm{Cre}}(\mathrm{j}, 1)$. Although there is a relative expansion of Tbr2+ cells in the mutant SVZ, most of them do not coexpress Ki67 (m,n). Arrowheads indicate colocalisation. At E17.5, expression of Lmo4 in the Wt can be seen in the medial and cingulate cortex (p); in Sip1fl/fl-Emx ${ }^{\mathrm{Cre}}$, expression in the cingulate cortex is highly reduced (q, arrowheads point to the cingulate cortex). In both cases a robust signal can be seen in the ganglionic eminences.

proportion of dividing cells express Tbr2. In other words, most abventricularly dividing cells in Sip1 mutants don't express Tbr2 (Fig7m,n). The characteristics of the increased number of non- dividing Tbr2+ cells is unknown; it is likely that they are either quiescent or have already exit the cell cycle.

In order to characterise the enhanced proliferation further, we looked for the expression of Brn2 in these cells. Brn2 is known to be expressed transiently in cells destined to become upper layer neurons while they are still located in the germinal zones. While in the wildtype cortex, we found most BrdU labeled cells to express Brn2 (since a significant percentage of upper layer neurons are still produced at E17.5), in mutant cortices, few expressed Brn2 at high levels while most showed weak to almost no Brn2 staining (Fig7k,1). All of the above features further corroborated our speculation that the enhanced proliferation seen at this stage is that of progenitors committed towards an astrocytic rather than a neuronal lineage.

Next, we looked at the expression of Lmo4 at E17.5 at rostral levels of the neocortex. Lmo4 is a transcription factor expressed in layers 2-3 and 5 at early postnatal stages (Bulchand et al., 2003). Further characterization suggested that in layer 5, it is expressed specifically in callosally projecting neurons, and is excluded from corticospinal motor neurons (Arlotta et al., 2005). In the wildytpe, we found strong expression in the medial and cingulate cortex (Fig7p). In contrast, in Sip1fl/fl-Emx ${ }^{\mathrm{Cre}}$, expression in the cingulate cortex seems highly reduced, while that in the medial pallium is unaltered (Fig7q). Expression of Lmo4 in the ganglionic eminences can be considered as an internal control 
(Fig7p,q) since the Emx1 promoter shows no activity in the subpallium, and hence, expression of Sip1 is not affected here. This observation is interesting considering the fact that at this stage, Satb2+ cells (that mostly project along the corpus callosum) are increased in numbers in the mutant cortex. Whether there is any correlation between reduced Lmo4 expression and increased Blbp/Olig2 expression in the cingulate cortex is yet to be determined.

\subsubsection{Premature production of layer 2-5 neurons in Sip1 conditional mutants}

A shift in the relative proportions of upper and deep layer neurons could arise due to several possible reasons, including changes in proliferation and apoptosis of young neurons. One other possible explanation would be changes in the timing of production of the different cell types during development as a consequence of precocious or delayed commitment of cortical progenitors towards specific neuronal lineages. Moreover, reduction of deep layers concomitant with expansion of upper layers, suggests that the latter are generated precociously at the expense of the former.

In order to test this hypothesis, we performed birthdating experiments involving BrdU pulses at specific timepoints during corticogenesis followed by chasing the pulsed brains to early postnatal stages. The scheme shown in Fig8a depicts how and when the pulse-chases were done. Our analyses showed that there is no change in the time course of production of Tbr1+ layer 6 neurons though the window of generation seems to expand over a larger time period (Fig8b,q,r). The fraction of cells born at E12.5 that express Tbr1 at P2 remains unaltered between wildtype and mutant cortices. However, the peak of generation of layer 2-5 neurons (Ctip2+, Foxp2+, Satb2+, Brn2+) is shifted to earlier stages of development. In wildtype cortices, Ctip2+ layer 5 neurons and Foxp2+ layer 3-5 cells are born primarily at E12.5-E13.5 (Fig8c,d). In Sip1 mutants, a much larger proportion of these cells are specified already at E12.5 (Fig8c,d). Similarly, Satb2+ layer 2-4 cells are born in the wildytpe primarily at E15.5; in mutants, a major fraction is generated already at E12.5 (Fig8e). Production of Brn2+ layer 2-4 neurons is distributed over a longer phase in wildtype embryos, whereas in mutant cortices, this phase is clearly moved to early stages (Fig8f). Shown in Fig8 are representative graphs (c-f) and co- immunostained images (g-j, $\mathrm{m}-\mathrm{p})$ illustrating the same. In wildtype cortices, cells born at E12.5/E13.5 despite being dispersed all over the cortical plate, settle mostly in the deep layers; in Sip1 mutant 

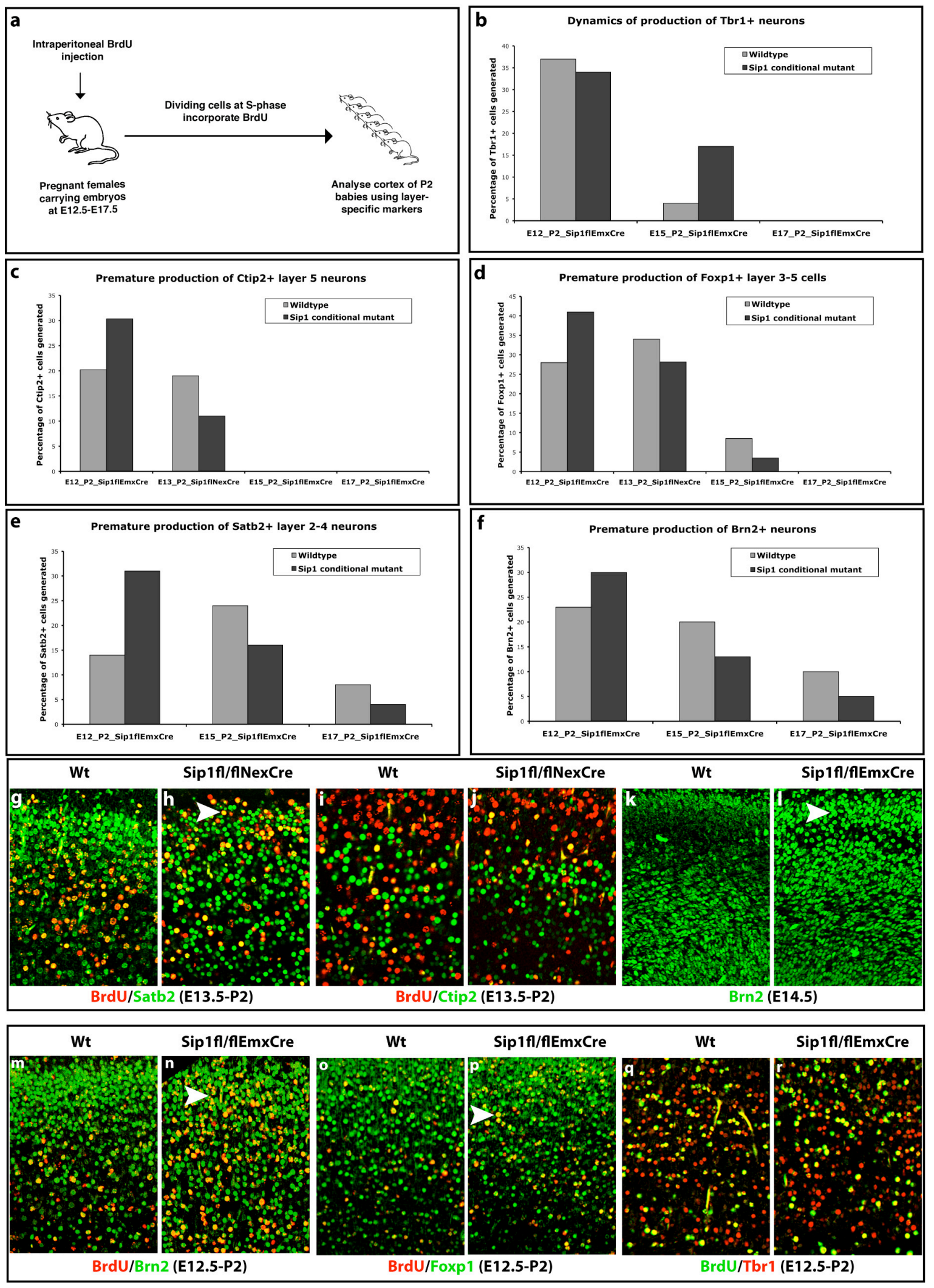

Fig8. Absence of Sip1 in the cortical plate induces precocious generation of layer 2- 5 cell types. The scheme of BrdU pulse- chase experiments to trace the fate of cells born at a certain stage of embryonic 
development is shown (a). Graphical representation of the dynamics of production of Tbr1+ layer 6 (b), Ctip2+ layer 5 (c), Foxp1+ layer 3-5 (d), Satb2+ layer 2-4 (e) and Brn2+ layer 2-4 (f) cells is shown; this indicates premature production of layer 2-5 neurons in the cortex, although the onset of neuronogenesis seems unchanged (b). Each graph depicts data from 1wildtype and 1 mutant; further quantification and statistical analysis on these data was done by our collaborators (Seuntjens and Huvlebroeck, University of Leuven). Representative double immunostained images show more cells labeled at E13.5 becoming Satb2+ neurons (g,h, arrowhead) and fewer becoming Ctip2+ (I,j) at P2 in Sip1fl/fl-Nex ${ }^{\mathrm{Cre}}$. Also, more cells labeled at E12.5 become Brn2+ (m,n, arrowhead) or Foxp1+ (o.p) at P2 in Sip1fl/fl-Emx ${ }^{\text {Cre }}$, while no such shift can be seen in the generation of Tbr1+ neurons (q,r). Additional evidence for the shift in upper layer neurogenesis comes from the premature expression of Brn2 in the cortical plate at E14.5 in Sip1fl/fl-Emx ${ }^{\mathrm{Cre}}$ (1, arrowhead), while no such expression can be detected in wildtype cortices at this stage (k).

cortices, however, not only do these cells settle at the upper layers of the $\mathrm{CP}$, they also express markers like Satb2 and Brn2, characteristic of upper layer neurons (Fig8g,h,m,n), and Foxp1, expressed in layers 3-5 (Fig8o,p). Furthermore, there seem to be fewer Ctip2+ cells born at E13.5 in the mutant (Fig8i,j), since their generation commences prematurely and takes place at high levels already at E12.5.

Considering the timing of production of Satb2+ and Brn2+ cells, it seems that in comparison to wildtype cortices, neurogenesis ends almost completely by E17.5 in Sip1 mutants, although a small percentage of cells born at this stage do differentiate into neurons (Fig8e,f).

As a consequence of the shift in cortical neurogenesis, Brn2+ cells destined to become upper layer neurons already start to appear in the lateral cortex in mutants at E13.5 while there are none observed in the wildtype at this stage. At E14.5 the premature expansion of Brn2+ population is more apparent (Fig8k,1). Also, a higher number of Er81+ layer 5 cells were found in the cortical plate, especially in the lateral region, in Sip1fl/fl-Emx ${ }^{\mathrm{Cre}}$ at E13.5. Altogether, deletion of Sip1 from the cortical plate seems to influence, non- cell autonomously, the fate of progenitors located in the VZ/SVZ, such that the precisely timed sequential generation of different cortical cell types is altered.

Eventually, at late postnatal stages, it seems that there are fewer neurons in the cortex of Sip1 conditional mutants than in wildtype (Fig11a-c). Together with the fact that neuronal production is shifted to earlier stages in these mutants, we expected a premature end of 
neurogenesis already at E15.5 when the first committed astrocytic precursors are born in both wildtype and mutants. In order to verify this, we looked at first at the number of cells born at E15.5; from the BrdU pulse chase experiments that were done to determine the birthdates of different cortical neuronal types, we concluded that there are fewer cells born at this stage (very few astrocytes, and the rest are neurons). Secondly, as mentioned before, we detected fewer Tbr2+ cells in the IZ in Sip1fl/fl-Emx ${ }^{\mathrm{Cre}}$ when compared with wildtype at E15.5 (Fig6m,n). Also, within the SVZ, there seem to be fewer Tbr2+ progenitors, though this observation has to be reconfirmed. The reduction in Tbr2+ cells in the IZ also suggests a decrease in neurogenesis at E15.5 in mutant cortices. This claim is supported by the following facts- first of all, our studies have shown that not all Tbr2+ cells are proliferating and that many cells continue to express Tbr2 even after they exit the cell cycle (further discussed in later sections); secondly, several independent reports have so far failed to establish the ability of Tbr2+ cells to generate glia, so that Tbr2 expression is fast becoming an indication of neuronal commitment. Altogether, the results of the birthdating experiments and the reduction in Tbr2+ cells in the IZ, strongly suggest that in Sip1 conditional mutants, there is indeed a premature end to neurogenesis, coinciding with precocious onset of gliogenesis (discussed below).

\subsubsection{Enhanced postnatal astrocytogenesis and premature specification of glial progenitors in the neocortex}

Since in both $\operatorname{Sip} 1 \mathrm{fl} / \mathrm{fl}-\mathrm{Emx}{ }^{\mathrm{Cre}}$ as well as $\mathrm{Sip} 1 \mathrm{fl} / \mathrm{fl}-\mathrm{Nex}^{\mathrm{Cre}}$ neurogenesis seems to end by E15.5, we suspected cortical progenitors at and after this stage to give rise to astrocytes, the next cell type generated in the cortex during development. Hence, we traced the fate of cortical progenitors of E15.5-E17.5 through BrdU pulse-chase experiments. We found that many cells born at E16.5 and E17.5 settled in upper layers of the cortical plate and expressed corresponding neuronal markers in wildtype cortices, whereas in Sip1 mutants, most of them ended up close to the ventricle in the region of the cingulate cortex. These cells did not express any upper layer neuronal marker. Interestingly, when we checked the expression of glial markers in the cortex at early postnatal stages, we detected atleast a five fold increase in GFAP expression in precisely the same region in Sip1 mutants (Fig9a,b,i) GFAP is considered a marker of terminal astrocyte differentiation (Dahl and Bignami, 1973). This suggested that progenitors at and after E15.5 were indeed getting committed towards an astrocytic fate, implying premature gliogenesis. We evaluated this further by 


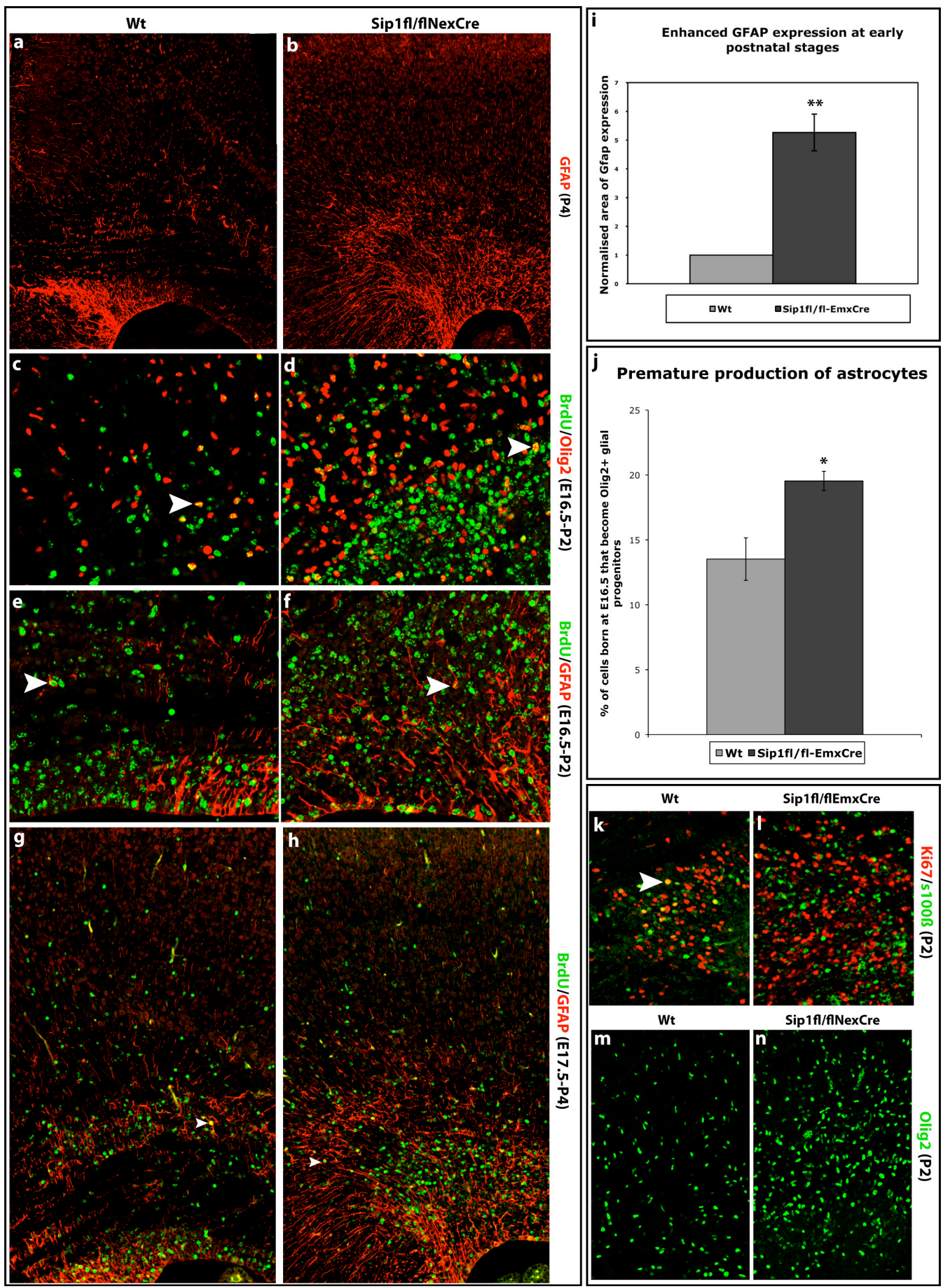

Fig9. Enhanced gliogenesis accompanied by premature specification of glial precursors in Sip1 depleted cortex. At early postnatal stages, immunostaining for astrocytic marker GFAP shows increased expression in both conditional mutants, especially in the cingulate cortex $(a, b)$. This effect was quantified in the cingulate cortex where 5-6 times higher GFAP expression was seen in Sip1 fl/fl-Emx ${ }^{\mathrm{Cre}}$ in comparison to 
the wildtype at P2 (i). Student's t-test was performed using $3 \mathrm{Wt}$ and 3 mutant brains from different litters. ${ }^{*} \mathrm{p}=0.002$. BrdU pulsed cells from E16.5 show increased coexpression of GFAP and Olig2 in Sip1fl/fl-Nex ${ }^{\mathrm{Cre}}$ (d,f, arrowheads) when compared to Wt (c,e). This effect has been quantified as the percentage of Olig2+ cells at P2 that are specified at E16.5 in Wt vs Sip1fl/fl-Nex ${ }^{\mathrm{Cre}}$ (j). Student's t-test was performed using $3 \mathrm{Wt}$ and 3 mutant brains. ${ }^{*} p=0.02$. Also, more cells born at E17.5 not only localize to the SVZ/IZ where most astrocytes are situated, but also express GFAP (g,h, arrowheads). The excessive astrocytes produced in Sip1 mutants are mature and not dividing, as shown by a double immunostaining for Ki67 and s100ß (k,1). Similar to GFAP, there is an increase in the number of Olig2+ cells as well, in Sip1fl/fl-Nex ${ }^{\mathrm{Cre}}(\mathrm{m}, \mathrm{n})$. Arrowheads indicate cells born presumably within the neocortex and not migrating from the ganglionic eminences. Error bars indicate S.E.M.

costaining BrdU labeled cells from E17.5 with GFAP at P2/P4. As expected, not only did we observe more cells born at E17.5 but also more of them expressed GFAP (Fig9g,h). We quantified this effect by double immunostaining for BrdU and Olig2. Olig2 is a bHLH transcription factor, which is not only essential for the development of motoneurons and oligodendrocytes in the spinal cord, but also plays a crucial role in deciding neuronal versus glial fate in cortical SVZ. It is known to be expressed transiently in immature astrocytes and VZ progenitors, and was shown recently to be important for white matter astrocyte development (Cai et al., 2007; Marshall et al., 2005). Our own observations in cortical slices cultured in vitro show a substantial amount of GFAP+ astrocytes coexpressing Olig2. Expectedly, we also detected increased numbers of Olig2 + cells at postnatal stages in Sip1fl/fl-Nex ${ }^{\mathrm{Cre}}$ (Fig9m,n). From the pulse- chase experiments, it was interesting to note that in wildtype mice, the commitment of cortical progenitors towards an astrocytic lineage already begins at E15.5. Very few BrdU labeled cells of E15.5 and E16.5, however, can be seen to coexpress GFAP or Olig2 at P2 (Fig9c,e). In both Sip1f1/fl$\mathrm{Emx}^{\mathrm{Cre}}$ as well as Sip1fl/fl-Nex ${ }^{\mathrm{Cre}}$, however, a significantly higher percentage of Olig2+ glial precursors and GFAP+ astrocytes are born at E16.5 (Fig9d,f). This effect has been quantified in Fig9j. The extent of colocalisation between BrdU and GFAP is difficult to quantify due to the pattern of staining of BrdU and GFAP (Fig9e-h). Hence, we preferred to analyse BrdU/Olig2 stainings for the purpose of quantification. We also compared the percentage of cells born at E17.5 that ended up as upper layer neurons in the postnatal cortex in wildtype and Sip1fl/fl-Emx ${ }^{\mathrm{Cre}}$ and found that this value seems to decrease in the latter (Fig8e,f). We could detect enhanced astrocytogenesis in the postnatal cingulate cortex until P17 in Sip1fl/fl-Emx ${ }^{\mathrm{Cre}}$.We next asked whether these excessive GFAP+ cells 


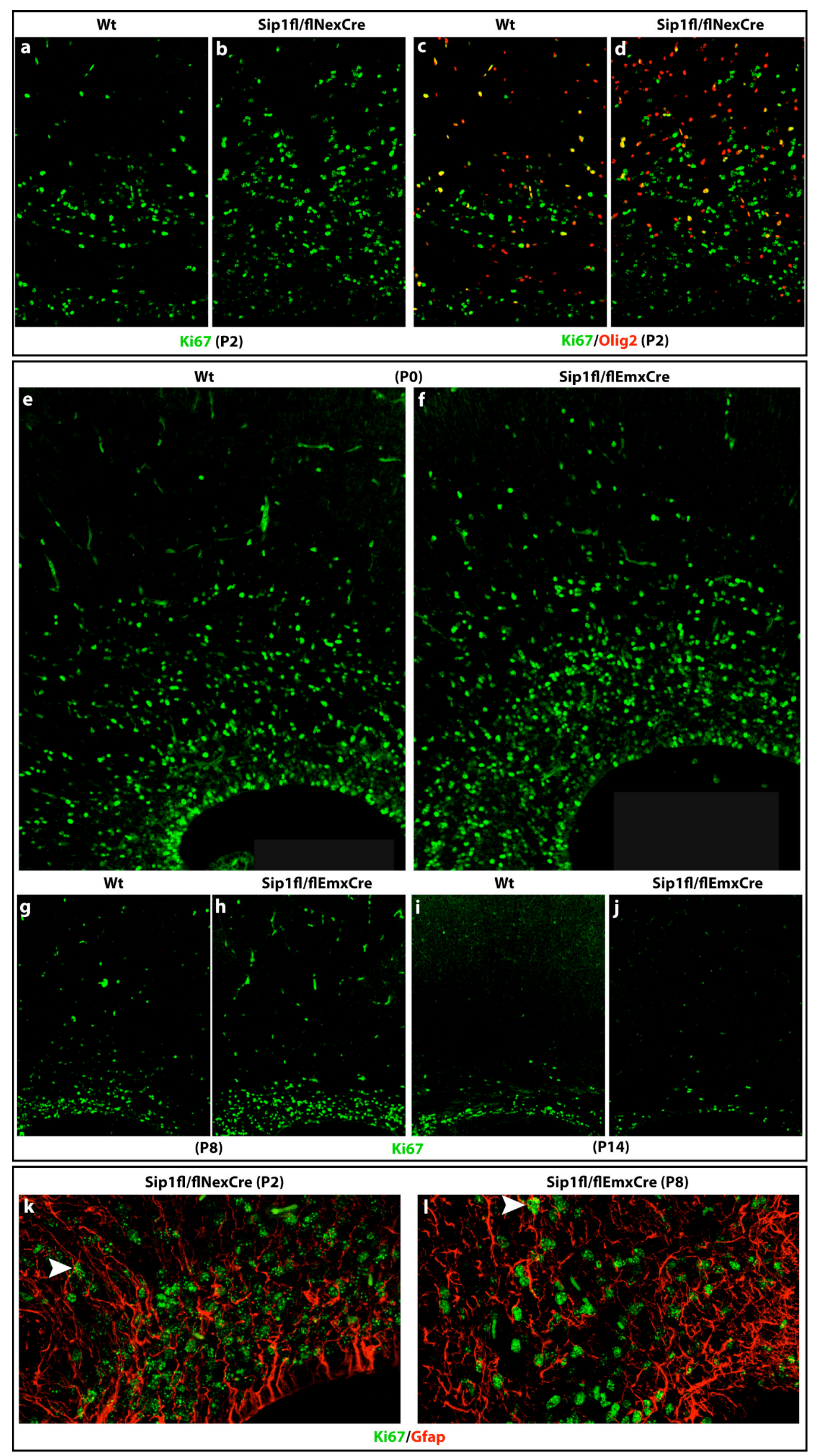


Fig10. Increased proliferation in the cingulate cortex continues to early postnatal stages in the absence of Sip1. Increased expression of Ki67 close to the ventricle can be seen in both conditional knockouts at P0 $(\mathrm{e}, \mathrm{f}), \mathrm{P} 2(\mathrm{a}, \mathrm{b})$, until P8 (g,h). At P14, this effect is no longer detected $(\mathrm{I}, \mathrm{j})$. At P2 in Sip1fl/fl-Nex ${ }^{\mathrm{Cre}}$, while some Ki67+ cells express Olig2 and/or GFAP, many don't (c,d,k,). At P8, again, both Ki67+GFAP+ as well as KI67+GFAP- cells can be seen around the ventricle in Sip1fl/fl-Emx ${ }^{\mathrm{Cre}}$ (1). Arrowheads indicate cells expressing Ki67 and GFAP.

were dividing or differentiated astrocytes. To address this question, we performed two sets of experiments- we did double immunostainings against, 1. The astrocytic marker s100ß and the proliferation marker Ki67 (Fig9k,1), and, 2. Ki67 and GFAP (Fig10k,1). While in case of the former, colocalisation was easy to assess because of the nuclear pattern of staining of both markers, in case of the latter, we imaged z-stacks followed by analysis as maximum projections (2D and $3 \mathrm{D}$ ). We believe that while the number of dividing astrocytes is not different between wildtype and Sip1 mutants, there are more differentiating astrocytes in the latter. It is important to note here that the appearance of GFAP astrocytes in both wildtype and Sip1 mutant cortices follows the same time course. The first astrocytes in the cortex are not detected before P2 in either the wildtype or the mutant. In other words, though there seems to be a precocious specification of astrocytic progenitors in the mutants, their differentiation into mature astrocytes is not shifted to earlier stages of development.

\subsubsection{Increased proliferation continues to early postnatal stages in the absence of Sip1}

We followed the enhanced proliferation at E17.5 to early postnatal stages, and found that the expression of Ki67 is higher in Sip1 mutants until at least P8 (Fig8a,b,e-h), which in turn coincides with the duration of high Sip1 expression in the neocortex (Fig21). The distribution of these cells around the ventricle is not different from the wildtype. While characterising these postnatally dividing cells in the mutants, we found that although some of them do express Olig2 and are possibly glial progenitors, there are many more that do not (Fig10c,d), and are probably undetermined progenitors, since we have no reason to believe that they could be neuronal precursors. As discussed above, we strongly suspect that neurogenesis ends prematurely in Sip1 mutants. Interestingly, at P14, this phenotype can no longer be observed; in fact, there seem to be fewer Ki67+ cells around the ventricle in Sip1fl/fl-Emx ${ }^{\mathrm{Cre}}$ cortices at this stage (Fig10i,j). As mentioned before, since the increase in proliferation spatially coincides with increase in GFAP expression, we determined the 
extent of colocalisation between the two. We detected both Ki67+GFAP+ dividing astrocytes as well as Ki67+GFAP- possibly uncommitted progenitors (Fig10k,1).

\subsubsection{Hampered migration of neurons born at and after E15.5}

As mentioned before, we suspected either defective migration or alterations in cell fate specification to be a cause of what seems as dispersed and incompact layering in the Sip1

mutant cortices (Fig11a-c). To begin with, we performed BrdU pulse- chase experiments to investigate into the two possibilities. We reasoned that if abnormal lamination is caused by cell fate defects, neurons labeled with BrdU at a certain stage of development should appear at comparable positions within the wildtype and mutant cortices. On the other hand, if neuronal migration is affected, they would end up occupying different positions. In Sip1 mutants, when BrdU was injected at E15.5 and chased to P2, we observed several cells

born at E15.5 that were located at the SVZ/IZ instead of upper cortical layers (Fig111,m). While some of these cells did express glial markers like GFAP or Olig2, most others expressed Brn2, expressed by young upper layer neurons before and during migration. Similarly, most cells born at E17.5 were found to be stuck in the SVZ/IZ/deep layers (Fig11n,o); again, many of these, especially those in the deep cortical layers, expressed Brn2. It therefore seems that radial migration of neurons born at and after E15.5 is hampered in Sip1 conditional mutants. On the other hand, when BrdU injections were done at E12.5/E13.5 and chased to P2, the labeled cells were found to predominantly occupy upper cortical layers in both mutants rather than deep layers. (Fig11p,q).

In order to investigate further into the potential cause of defective migration in Sip1 mutant cortices, we checked the expression of several molecules involved in regulating this process either directly or indirectly. Doublecortin (Dcx) is known to be essential for radial migration especially of upper cortical plate neurons. Both knock down of Dcx as well as double mutation of Dex and Dclk leads to abnormal migration in the cortex (Bai et al., 2003; Deuel et al., 2006). We did not find any obvious differences in the pattern of expression of Dcx in Sip1 mutants. At E15.5, in both wildtype and Sip1fl/fl-Emx ${ }^{\mathrm{Cre}}$, strong Dcx expression was seen in the upper part of the cortical plate, while relatively weaker expression was detected in the deeper parts of the CP, basal SVZ and IZ (Fig12i,j). 


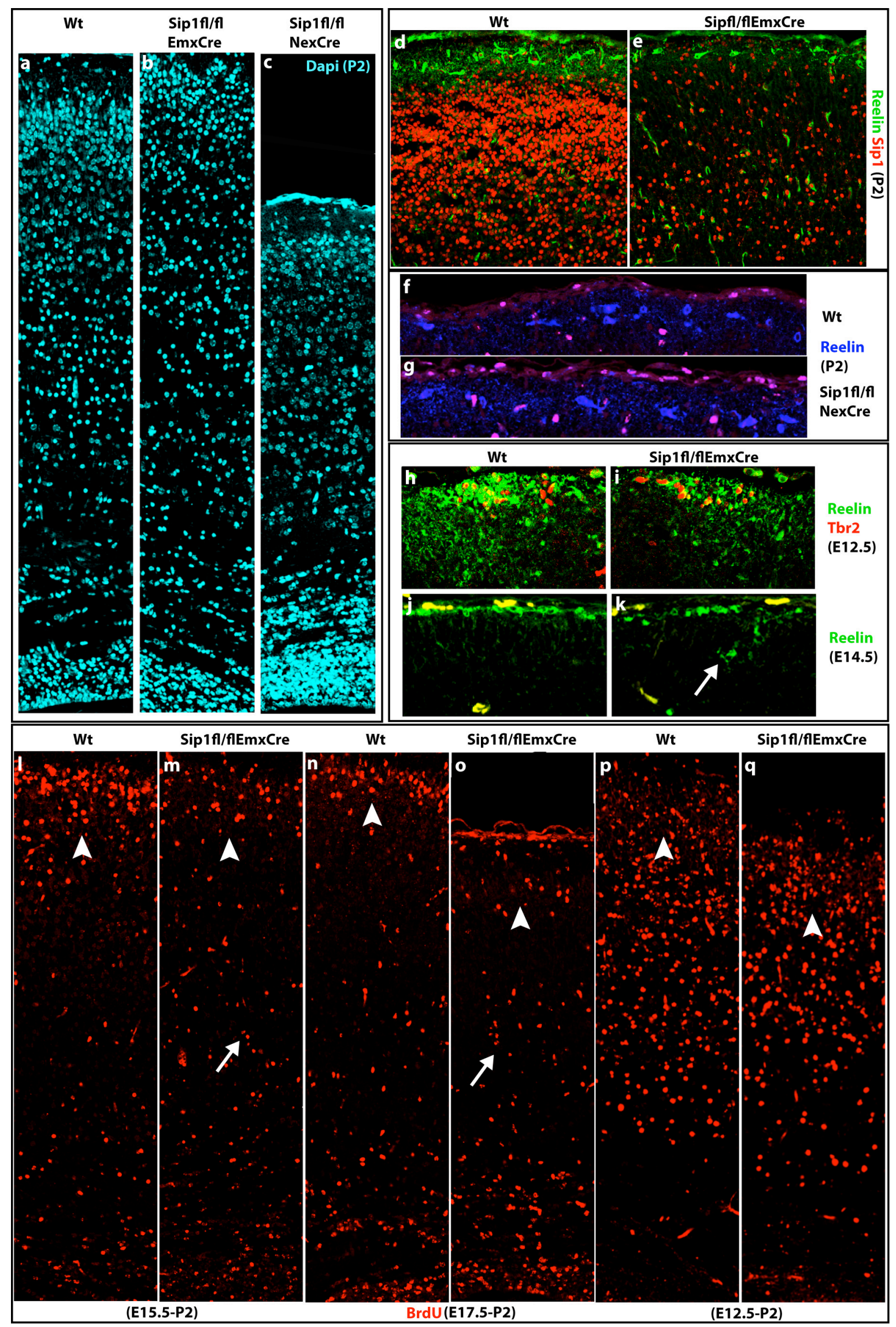


Fig11. Young neurons born after E15.5 do not migrate properly to their final destination within the cortical plate; defects in Reelin expression might not be the cause. Staining of Wt and mutant cortices for DAPI clearly shows the disorganized stratification of cortical layers at P2 (a-c). Expression of Reelin within the marginal zone is reduced at P2 in $\operatorname{Sip} 1 \mathrm{fl} / \mathrm{fl}-\mathrm{Emx}^{\mathrm{Cre}}(\mathrm{d}, \mathrm{e})$ but not in Sip1fl/fl-Nex ${ }^{\mathrm{Cre}}$ (f,g). In Sip1fl/fl$\mathrm{Emx}^{\mathrm{Cre}}$ several Reelin+ cells can be seen within the cortical plate that express Sip1 (e). Co-expression of Reelin and Tbr2 in the putative piriform cortex at E12.5 is reduced in Sip1fl/fl-Emx ${ }^{\mathrm{Cre}}$ (h,i). Ectopically located Reelin+ cells (arrow) can be seen within the cortical plate in Sip1fl/fl-Emx ${ }^{\mathrm{Cre}}$ at E14.5 (j,k). BrdU pulse- chase experiments show that neurons born at and after E15.5 fail to migrate to the appropriate location (arrowheads) and are instead stuck in the IZ/deep layers (arrows). Shown here are E15.5 to P2 chase $(1, \mathrm{~m})$ and E17.5 to P2 chase $(\mathrm{n}, \mathrm{o})$ in wt and Sip1fl/f1-Emx ${ }^{\mathrm{Cre}}$. Corroborating the premature specification of upper layer neurons, cells born at E12.5 settle at upper layers in Sip1fl/fl-Emx ${ }^{\mathrm{Cre}}$ (q, arrowhead) by P2, while in Wt they are dispersed mostly through layers 4-6 (p).

Reelin is a secreted signaling molecule that ensures proper migration of young neurons along radial glial fibers. Mutations in Reelin, its receptors VLDLR and ApoER2, and downstream effectors (Dab1) all lead to similar phenotypes that involve a migration delay leading to abnormal neuronal positioning and inverted cortical layers (Tissir and Goffinet, 2003). In Sip1fl/fl-Emx ${ }^{\mathrm{Cre}}$ we did observe a decrease in Reelin expression in comparison to the wildtype (Fig11d,e). Besides the Cajal-Retzius cells of the MZ, several Reelin+ cells were also observed within wildtype and mutant cortical plate. Interestingly, in these mutants almost all Reelin+ cells of the cortical plate were the ones that continued to express Sip1, either because they escaped Cre- mediated recombination, or because they are interneurons that were born in the ganglionic eminences and that migrated tangentially into the neocortex (Fig11d,e). When we checked Reelin expression at earlier stages of development, we detected two major phenotypic effects; 1. At E12.5, Reelin expression is restricted mostly to the putative piriform cortex where they coexpress Tbr2. In Sip1fl/fl$\mathrm{Emx}^{\mathrm{Cre}}$ there were fewer Reelin+ cells in this region (Fig11h,i); 2. At E14.5 cells secreting Reelin are localised to the marginal zone. In Sip1fl/fl-Emx ${ }^{\mathrm{Cre}}$ not only are these cells fewer in number, many of them were localised ectopically in the cortical plate (Fig $11 \mathrm{j}, \mathrm{k})$. It therefore seems that absence of Sip1 in cortical progenitors leads to a reduction in levels of Reelin, which might in turn lead to the dispersion of cortical layers. However, when Sip1 is ablated from only the postmitotic cells of the cortex ( $\left.\operatorname{Sip} 1 \mathrm{fl} / \mathrm{fl}-\mathrm{Nex}{ }^{\mathrm{Cre}}\right)$, Reelin expression remains unchanged (Fig11f,g), suggesting that the migration delay in Sip1 mutants is either due to a deficiency in effectors downstream of Reelin or due to a deficiency in alternative pathways like the p35/Cdk5 mediated control of neuronal migration (Gilmore et al., 1998; Ohshima et al., 2001). Alternatively, such a migration defect could also be caused by a 

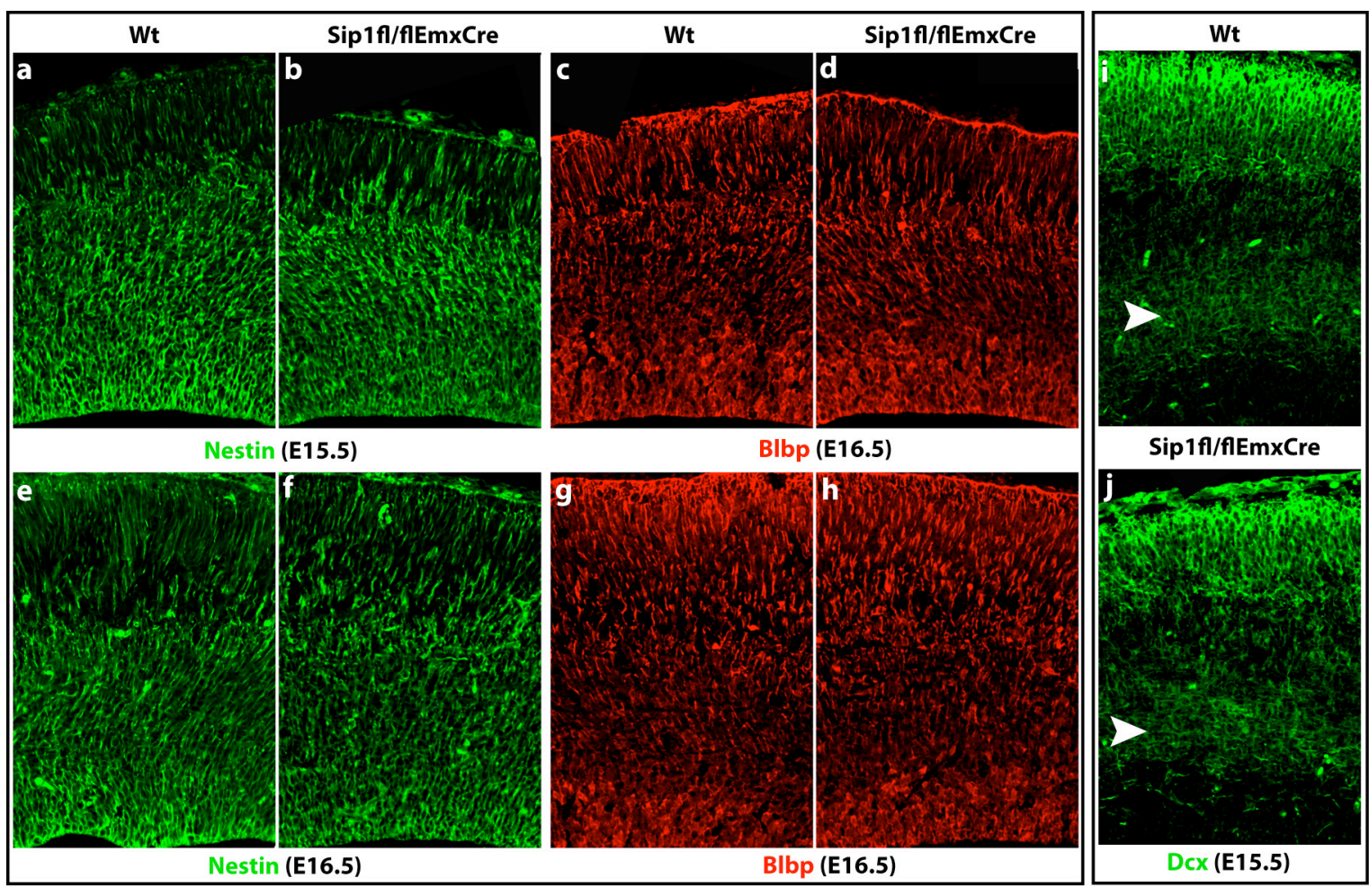

Fig12. Cortical deletion of Sip1 does not affect radial glial processes or the expression Doublecortin, precluding them as a cause of hampered migration. Expression of Nestin and Blbp is unaltered at E15.5 (a-d) and E16.5 (e-h). Strong expression of Dcx can be seen in the cortical plate close to the pial surface in both Wt (i) and Sip1fl/fl-Emx ${ }^{\mathrm{Cre}}$ (j) at E15.5; there is also weaker expression in the basal SVZ/IZ, which also seems unaffected in the mutant cortex (arrowhead).

disruption in radial glial processes that are often used as a scaffold to migrate on, by several young neurons until they reach their final position in the cortical plate (Nadarajah, 2003). In order to check the integrity of radial glial processes in Sip1 mutants, we labeled them using radial glial markers Nestin and Blbp (Hartfuss et al., 2001; Hockfield and McKay, 1985). We detected no abnormalities in the morphology of these processes in Sip1fl/fl-Emx ${ }^{\text {Cre }}$ at E15.5/E16.5 (Fig12a-h). Radial glial processes, as the name suggests, extend radially from the apical to the pial surface. Although the staining for Nestin is clearly cytoplasmic, Blbp expression can often be detected in the cell bodies of radial glial cells located in the VZ. 


\subsection{Sip1- mediated signaling in the neocortex}

\subsubsection{Molecular targets of Sip1 in the cortex}

In order to investigate the molecular mechanism(s) behind the diverse range of phenotypic characteristics observed in Sip1fl/fl-Emx ${ }^{\mathrm{Cre}}$ and Sip1fl/fl-Nex ${ }^{\mathrm{Cre}}$, we set out to first determine the genes whose expression is either directly or indirectly controlled by Sip1 levels in the cortex. We performed microarray based comparison of gene expression between wildtype and mutant cortex tissue at E13.5, E14.5 and E16.5. We reasoned that while the former two developmental stages would be crucial in premature upper layer production, the latter would be critical for precocious gliogenic specification of cortical precursors. Since Sip1 is known to be primarily a transcriptional repressor, we focused on those genes whose expression was upregulated in Sip1 conditional mutants. Also, since it seems that deletion of Sip1 in postmitotic cells alone ( $\left.\operatorname{Sip} 1 \mathrm{fl} / \mathrm{fl}-\mathrm{Nex}{ }^{\mathrm{Cre}}\right)$ is sufficient to alter progenitor fate, we hypothesised that Sip1 might actually mediate its function via secreted growth factors and/or cytokines. Hence, we further focused on these genes and those encoding the corresponding receptors. The expression levels of the most promising candidates were further characterised by semi-quantitative PCR (SQ-PCR) using primer pairs targeted to the last two exons at the 3 ' end of the mRNA (Fig13b-e). A list of the most promising targets is shown in Fig13a. Of these, we decided to explore the role of Neurotrophin-3 and Fgf9 in particular, since both are secreted growth factors. Next, we confirmed upregulation of neurotrophin-3 and Fgf9 in Sip1 mutants by in situ hybridisation, which was done in collaboration with Dr.Eve Seuntjens and Prof. Danny Huylebroeck at the University of Leuven, Belgium. Interestingly, we found that the onset of expression of these two factors is shifted to one day earlier in Sip1fl/fl-Nex ${ }^{\mathrm{Cre}}$. This in turn led us to speculate that the premature generation of upper layer neurons in the absence of sufficient levels of Sip1 could be mediated via NT3, while the precocious astrocytogenesis could be mediated via Fgf9. We next performed in vitro experiments to prove the same. 
a

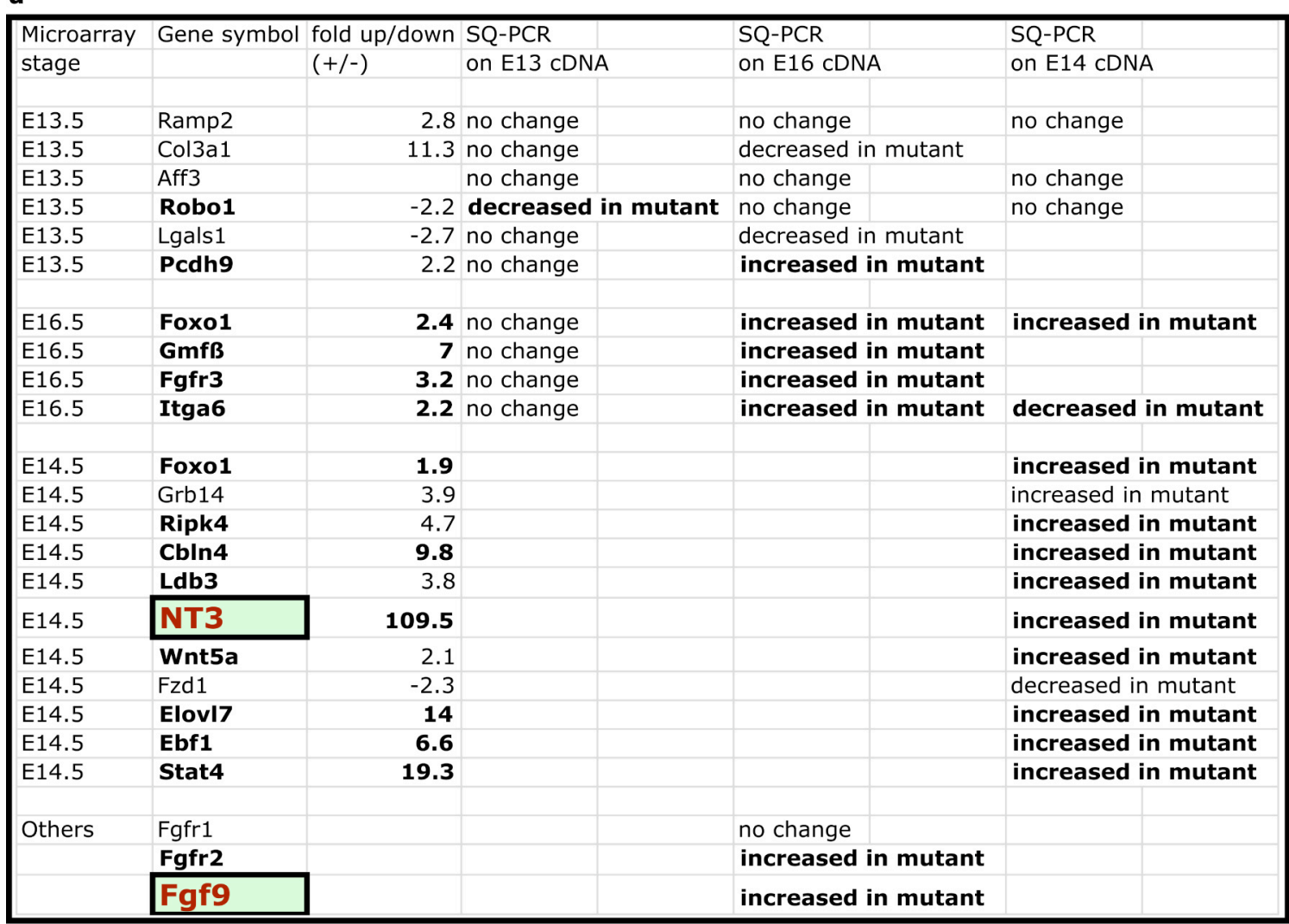
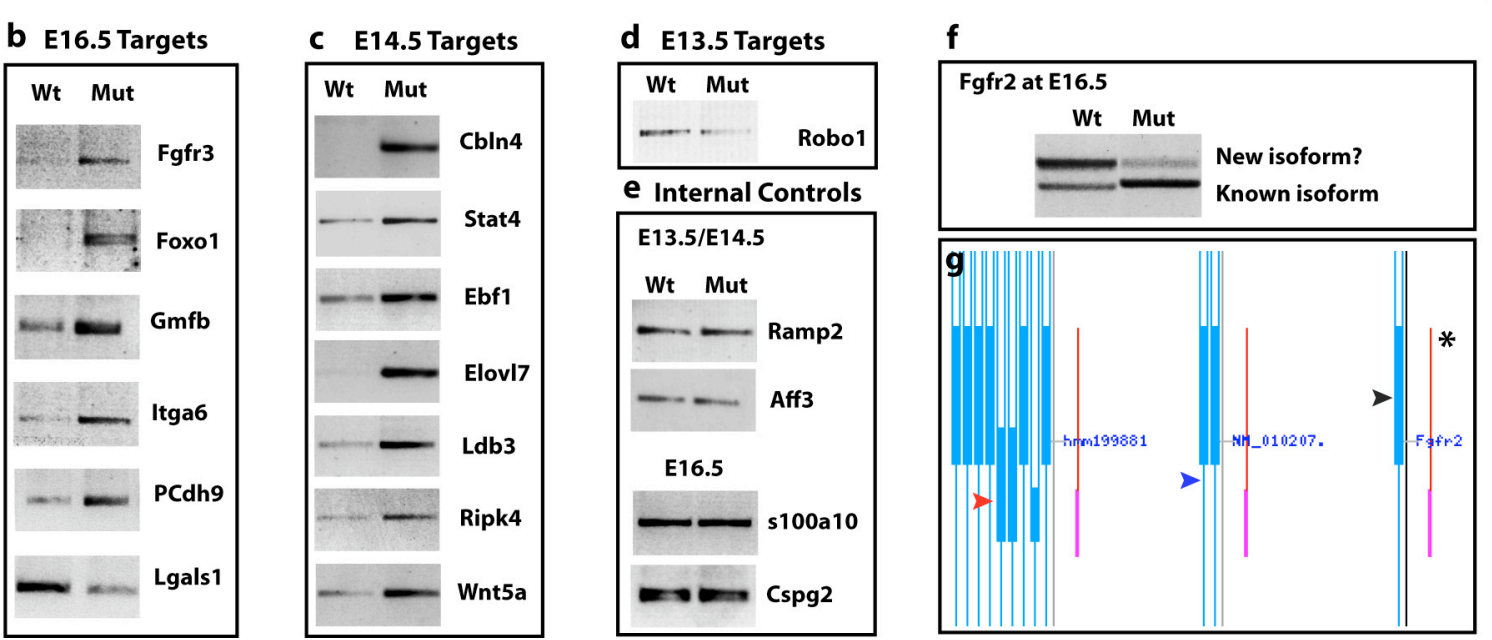

Fig13. Sip1 regulates expression of several genes in the developing neocortex, including a newly identified isoform of Fgfr2. The most promising targets of Sip1 in the neocortex (based on microarray and semi- quantitative PCR (SQ-PCR) based comparison of gene expression) at E13.5, E14.5 and E16.5 are summarized (a). The result of SQ-PCR for genes whose expression is strongly up-/downregulated in Sip1fl/fl-Emx ${ }^{\mathrm{Cre}}$ at E16.5 (b), E14.5 (c) and E13.5 (d) is also shown. Some genes whose expression remains unaltered were used as internal controls (e). SQ-PCR for Fgfr2 cDNA revealed 2 PCR products- the expected isoform, which is upregulated, and an as yet unidentified splice variant in the cortex, which is downregulated (f). An alignment of the new isoform against the mouse genome database on MapViewer suggests the existence of an unannotated exon within the 3' intronic sequence of Fgfr2 gene (g). * is the sequence of the SQ-PCR product that aligns with the last coding exon (black arrowhead) and partially with an intronic 
sequence (blue arrowhead) of Fgfr2 gene. The sequence of the new variant also matches a computationally predicted exon (red arrowhead). The data shown in $\mathrm{f}$ and $\mathrm{g}$ was procured and analysed together with Marten Kuscher.

\subsubsection{Fgf signaling is affected in Sip1 mutant cortices- likely to be mediated via Fgf9 and Fgfr2/Fgfr3}

Besides Fgf9, we identified Fgfr3 as a putative Sip1 target through SQ-PCR at E16.5 (Fig13b). As reported earlier, Fgfr3 expression cannot be detected at this stage in the cortex (Inglis-Broadgate et al., 2005); however, in the cortical tissue isolated from Sip1fl/fl-Emx ${ }^{\mathrm{Cre}}$ we could already detect a considerable level of the corresponding cDNA. We also detected an increase in the expression of Fgfr2 (Fig13f). To our surprise, we also detected an additional PCR product in the semi- quantitative PCRs that were done to amplify the 3' end of Fgfr2 cDNA. Together with Marten Kuscher in our lab, we sequenced this unknown fragment, and BLASTed and aligned it against the mouse genome database in MapViewer on NCBI. We found that this PCR product contains an unannotated sequence that aligns with an intronic sequence of the Fgfr2 gene (Fig13g). It also aligns with a computationally predicted exonic sequence (Fig13g). It therefore seems to be an as yet unknown alternatively spliced isoform of this receptor in cortical tissue.

Interestingly, its levels were actually reduced in mutant cortices (Fig13f,g). Since all three Fgf receptors have been implicated in corticogenesis, and since both Fgfr1 and Fgfr2 are expressed in the cortical VZ at E16.5, we next looked at the levels of Fgfr1 in Sip1 mutant cortices. We did not detect any changes with regard to the cDNA levels. Furthermore, in order to assess possible genetic interactions between Sip1 and Fgfr1, we generated a Sip1/Fgfr1-Emx ${ }^{\text {Cre }}$ double conditional mutant, and analysed the extent of proliferation at $\mathrm{P} 1$. It is important to reiterate that in Sip1 fl/fl-Emx ${ }^{\mathrm{Cre}}$ there is an increase in proliferation at the SVZ of the cingulate cortex from E17.5 onwards. An immunostaining for proliferation marker PCNA indicated a much higher number of dividing cells in Sip1fl/fl/Fgfr1fl/fl$\mathrm{Emx}^{\mathrm{Cre}}$ double mutants in comparison to wildtype and Fgfr1fl/fl- $\mathrm{Emx}^{\mathrm{Cre}}$ where the extent of proliferation was similar to that in the wildtype (Fig14e-g). Therefore, neither does lack of Fgfr1 itself influence cortical cell division at birth, nor does its absence in Sip1 mutants rescue the enhanced proliferation seen in the latter (Fig14e-g). 


\subsubsection{Exogenous application of Fgf9 to wildtype cortical slices induces proliferation of}

Olig2+ glial precursors

In order to check whether premature upregulation of Fgf9 in Sip1 conditional mutants is indeed the cause of excessive gliogenesis in these mutants, we cultured E16.5 wildtype cortical slices in the presence and absence of Fgf9 in the culture medium. After two days in culture, the slices treated with Fgf9 were found to contain 2.7 times more Olig2+ cells in comparison to control slices (Fig14a-d). It is interesting to note that the excess of Olig2+
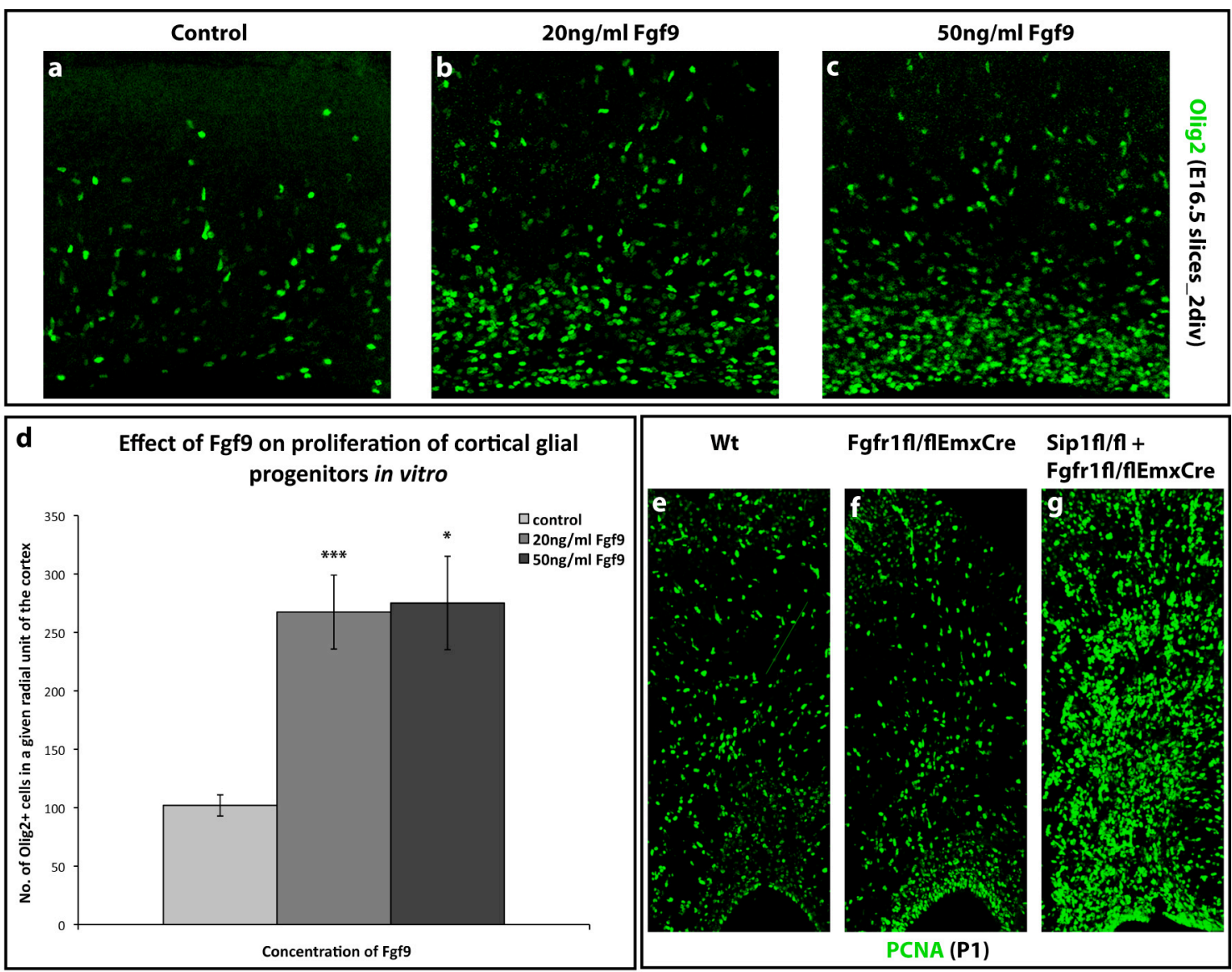

Fig14. Fgf9 can induce increased production of Olig2 expressing glial progenitors in vitro. E16.5 Wt slices treated with different concentrations of Fgf9 and cultured for 2div were stained for Olig2. Control slices (a), and slices treated with 20ng/ml Fgf9 (b) and 50ng/ml Fgf9 (c) are shown. The number of Olig2+ cells under different conditions was quantified over the entire radial unit of a specified width, over 3 independent experiments using slices from 5 to 8 wildtype brains per experiment. There were 2.7 times more Olig2 + cells in Fgf9 treated slices in comparison to control slices (D). Student's t-test: $* * * p=0.0001$, ${ }^{*} \mathrm{p}=0.01$. Error bars indicate S.E.M. Immunostaining for PCNA at P1 in Wt (e), Fgfr1fl/fl-Emx ${ }^{\mathrm{Cre}}$ (f) and Sip1fl/fl-Fgfr1fl/fl-Emx ${ }^{\mathrm{Cre}}(\mathrm{g})$ shows neither a difference in the number of dividing cells in the absence of Fgfr1, nor a rescue of Sip1- mediated increase in early postnatal proliferation. 

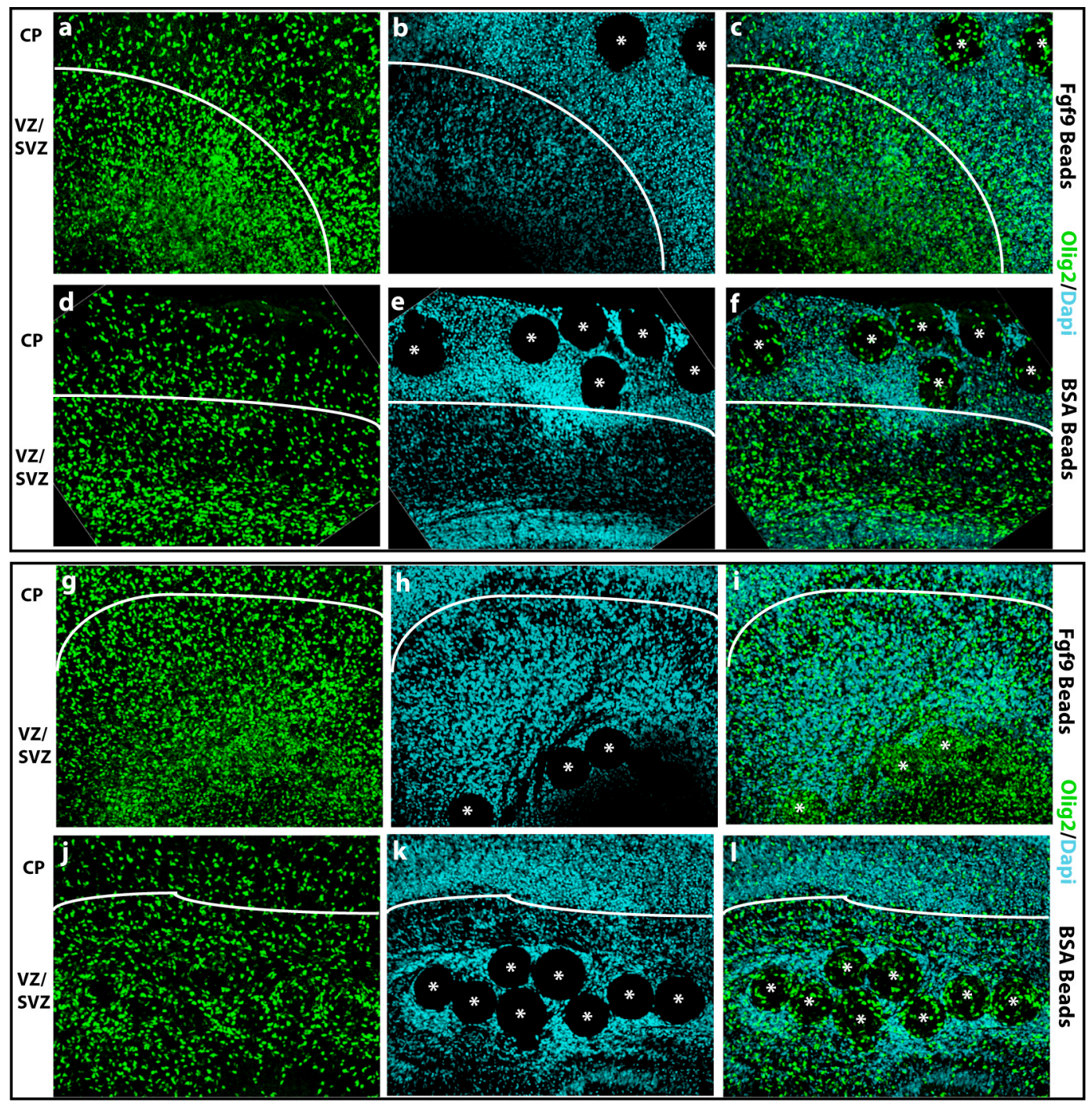

$m$ Effect of FGF9 coated beads on gliogenesis in E17.5 Wt cortical slices

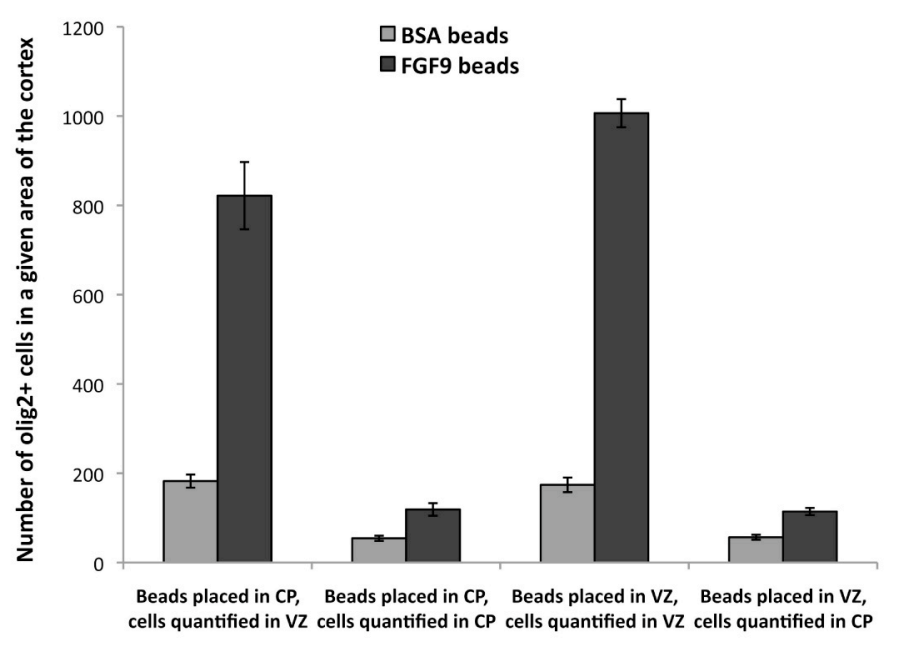

Fig15. Localised application of Fgf9 in the cortical plate can induce gliogenic proliferation in the ventricular zone, suggesting existence of Fgf receptors on radial glial processes. Fgf9 (a-c, g-i) and BSA (d-f, j-1) coated beads were placed in either the cortical plate (a-f) or the VZ/SVZ (g-1) of E17.5 wildtype 
slices and cultured for $2 \mathrm{div}$. Shown here is staining for Olig2 and nuclei (DAPI). The increase in the number of Olig2 + cells under the four different conditions is quantified within defined regions of either the cortical plate or the VZ/SVZ (m). Atleast 10 slices per condition collected randomly from 5 embryos were analysed. Error bars indicate S.E.M.

cells were mostly localised close to the ventricle, although Fgf9 was homogenously present throughout the culture medium. We hypothesised that Fgf9 binds to Fgf receptors that are expressed either on the processes or on the surface of the cell bodies of radial glial cells; this in turn leads to activation of a signaling cascade that most likely alters gene expression within these cells leading them to switch fate towards a glial lineage and start expressing Olig2. For those that are already committed towards an Olig2-lineage, the Fgf9 signaling probably causes them to divide further and increase their pool size. This is probably why we see increased Olig2 expression at the ventricle on Fgf9 treatment, since radial glial cells are located in the VZ. In order to investigate this mechanism further, we decided to study the consequences of localised application of Fgf9 to cortical slices. For this, Fgf9 coated Affigel Beads were placed either in the CP or the VZ/SVZ of E16.5/E17.5 wildtype slices. We reasoned that if Fgf9 signals to progenitors via radial glial processes, placing Fgf9coated beads in the cortical plate should result in activation of Fgf receptors localized on them, leading to a signaling cascade that would increase the number of Olig2+ cells at the germinal zone. The second possible scenario would be that Fgf9- mediated signaling does not take place via radial glial processes, but instead through direct diffusion of Fgf9 to the germinal zone where it would bind to the corresponding receptors and induce enhanced astrocytic commitment/proliferation. In this case, placing Fgf9- coated beads in the CP should not alter the numbers of Olig2+ cells at the VZ/SVZ. In both scenarios, however, we would expect implantation of Fgf9- coated beads in the VZ/SVZ to induce gliogenic proliferation in that region. As a control we placed BSA-coated beads in the $\mathrm{CP}$ and the VZ/SVZ. We found that after two days in culture, slices beaded with Fgf9- coated beads contained much more Olig2+ cells at the ventricle, irrespective of the location of Fgf9 source (Fig15a-c, g-i). Olig2 expression in control slices implanted with BSA- coated beads was as expected for this stage of development (Fig15d-f, j-1). Therefore, it seems that Fgf receptors are indeed distributed on the processes as well as on the cell bodies of radial glial cells. 


\subsubsection{Exogenous application of Neurotrophin-3 to wildtype cortical cells/explants is not sufficient to change the proportion of upper versus deep layer neurons}

Upregulation of NT3 in Sip1 mutant cortices coincides with the premature production of upper layer neurons. NT3 is known to bind TrkC receptors (Huang and Reichardt, 2003), which were shown to be expressed throughout the neocortex at high levels at E14.5 and earlier (unpublished data from Dr. Eve Seuntjens, University of Leuven). We speculated that if wildtype cortical cells/tissue are exposed to high levels of NT3 at stages earlier than the normal onset of expression, they might trigger a signaling cascade that might in turn mediate the cell fate switch of early cortical progenitors towards an upper layer lineage. To check if this is indeed the case, we performed a set of in vitro experiments. At first, E12.5 cortices were dissociated into single cells and cultured in a medium containing high concentrations of NT3 for 2-3 div. We then checked the proportion of cells expressing deep layer markers Ctip2/Sox5/Foxp1 versus those expressing upper layer markers Satb2/Brn2. We did not find any significant differences. As a corollary, we used a function blocking anti- NT3 antibody to block endogenously produced NT3 in wildtype and Sip1 mutant cortical cells, and again checked the ratio of different cell types. Since NT3 is not expressed endogenously in wildtype cortices at E12.5, we did not expect any changes in the cell numbers in the presence of the anti- NT3 antibody. However, the latter might block the upregulated NT3 in Sip1 mutants, and rescue the effect of premature differentiation into layer 5 and layer 2-4 neurons. Although there seems to be a decrease in the percentage of Satb2+ and Ctip2+ cells generated by mutant cortical progenitors in the presence of anti- NT3 antibody, this decrease was not statistically significant (Fig16h-k). Hence, we decided to revert to cortical explants where the tissue structure (radial glial processes, extracellular matrix, cell- cell contact, microenvironments etc.) is maintained, and is therefore more similar to conditions in vivo (Fig16a.b,f,g). We isolated entire cerebral cortices (either including or excluding the basal ganglia) of E12.5-E13.5 wildtype and Sip1 conditional mutants, and placed them in culture for 2-3div. In this case, we calculated the ratio of Satb2+:Ctip2+ cells at different concentrations of NT3. We reasoned that if NT3 alone can indeed induce cortical progenitors to differentiate into upper layer neurons, the given ratio should increase in its presence. However, even when added at concentrations of upto $600 \mathrm{ng} / \mathrm{ml}$, NT3 did not change this ratio whatsoever (Fig16c). We also found no differences when NT3 was added to dissociated E13.5 cortical cells. One of the possible reasons why we do not see any effect of NT3 on cell fate is that the local concentration of 


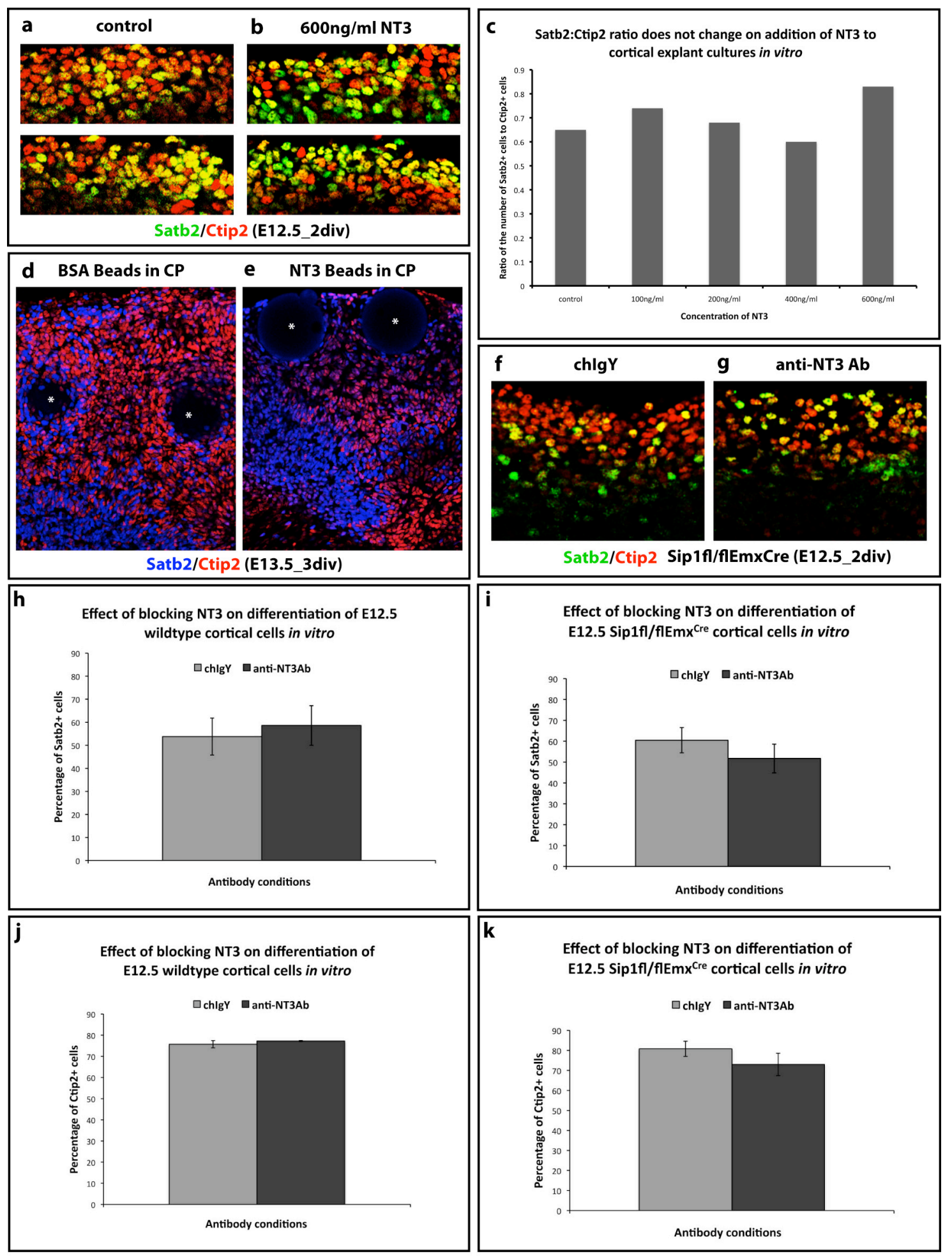

Fig16. NT3 alone cannot induce changes in proportions of upper layer vs deep layer neurons in vitro.

E12.5 cortical explants cultured for 2div with and without exogenous NT3 were immunostained for Satb2 and Ctip2 (a,b). No obvious differences can be seen in the relative proportions of the two cell types (c). NT3coated and BSA- coated (as control) beads were placed in the cortical plate on E13.5 wildtype slices and cultured for 3div, followed by staining for Satb2 and Ctip2 (d,e). Again, no differences were observed. As a corollary, function- blocking anti- NT3 antibody was used on E12.5 cortical explants to block endogenous activity in Wt and Sip1fl/fl-Emx ${ }^{\mathrm{Cre}}$ slices, cultured for 2div. Chicken IgY antibody was used as control. 
Mutant slices stained for Satb2 and Ctip2 are shown (f,g). The relative proportions of the two cell types seem unaffected. The same approach was also used on dissociated E12.5 cortical cells (Wt and Sip1fl/fl$\mathrm{Emx}{ }^{\mathrm{Cre}}$ ) cultured in vitro in the presence/ absence of the antibody for 2div. The percentage of cells expressing Satb2 (h,i) and Ctip2 (j,k) was quantified. In Sip1 fl/fl-Emx ${ }^{\mathrm{Cre}}$ cell culture, although there seems to be a trend towards decreased layer 5 (Ctip2+) and layer 2-4 (Satb2+) differentiation in the presence of the anti- NT3 antibody, this difference was not statistically significant (i,k).

NT3 that cells in the mutant cortex are exposed to is very high and that adding NT3 to the culture medium is not sufficient to reach such high levels in vitro. Therefore, in the final set of experiments, we coated Affigel beads with NT3 and placed them on E13.5 wildtype cortical slices and cultured them for 3div. This was followed by immunostaining for Satb2, Ctip2 and Sox5 (Fig16d,e). There does not seem to be any obvious difference in the proportion of any of these cell types. It therefore seems that NT3 alone cannot influence cell fate switch in cortical progenitors but that it might act in combination with other cytokines/ growth factors.

\subsubsection{BMP signaling is activated in ectopically dividing cells in Sip1 conditional mutants at E17.5}

Next, we tried to investigate which gliogenic signaling pathway(s) is/are activated as a consequence of deleting Siplin the differentiating field of the cortex. It was shown more than a decade ago that BMPs can induce SVZ progenitors to commit to an astroglial lineage (Gross et al., 1996). Recent work has shown that BMPs can induce neuroepithelial cells to become astrocytes (Bonaguidi et al., 2005; Nakashima et al., 2001; Yanagisawa et al., 2001). Since our results indicate enhanced proliferation of presumably astrocytic precursors at E17.5 in Sip1fl/fl-Emx ${ }^{\mathrm{Cre}}$ and $\mathrm{Sip} 1 \mathrm{fl} / \mathrm{fl}-\mathrm{Nex}^{\mathrm{Cre}}$, as well as precocious commitment to astrocytic lineage at E16.5, we decided to test whether increased BMP activity plays any role in inducing this phenotype. To detect this signaling pathway, we immunostained wildtype and mutant cortices for phosphorylated Smad1/5/8. It is known that transcription factors Smad1/5/8 are downstream effectors of the BMP signaling pathway that get phosphorylated on activation of BMP receptor (Mehler et al., 1997). It is interesting to note here that this staining can also be used as a marker for progenitors committed towards an astrocytic lineage since it has been well established that BMP activity at late stages of corticogenesis will lead cells to differentiate into astrocytes 


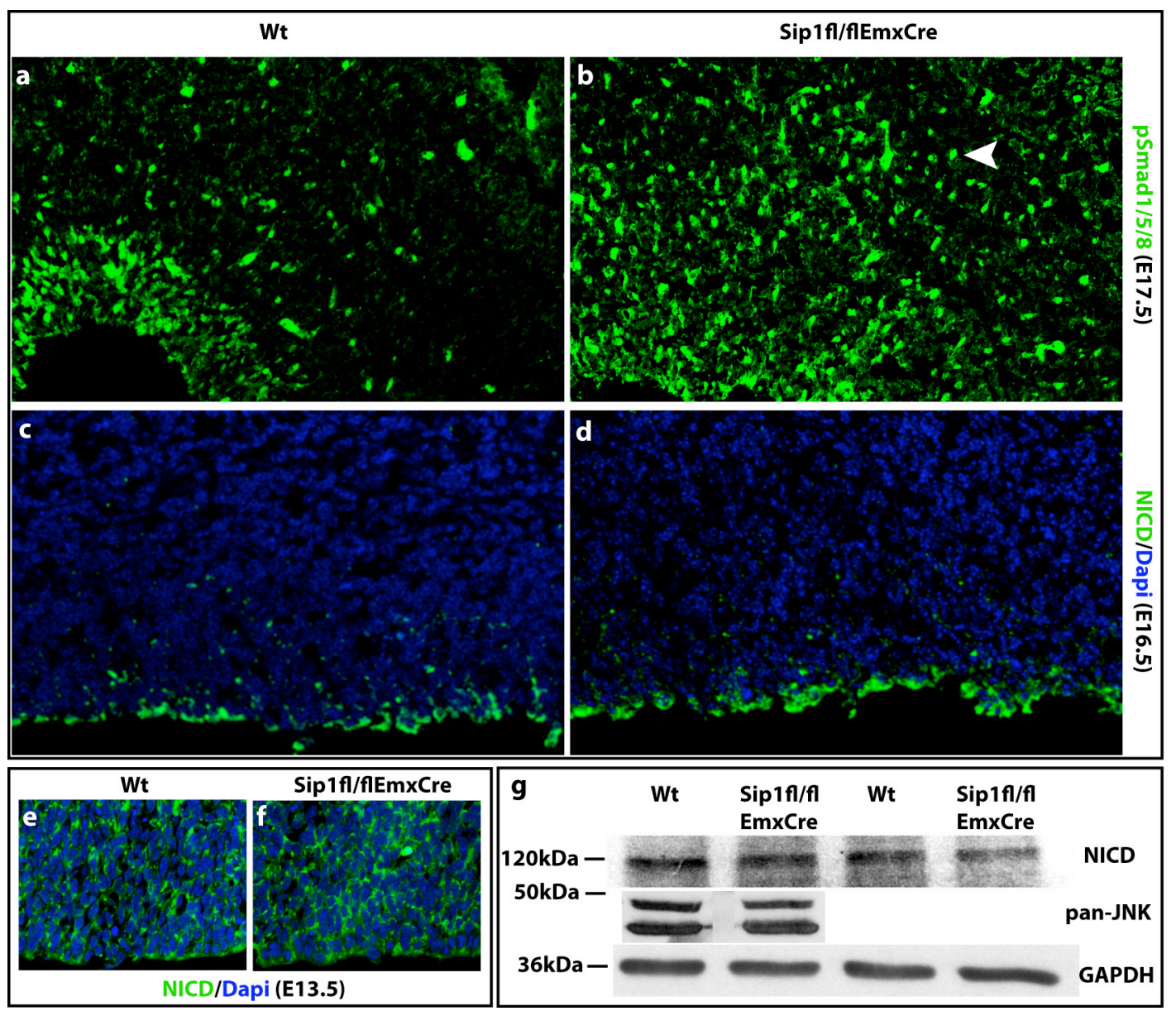

Fig17. Strong BMP activity is seen in abventricularly dividing cells of Sip1fl/fl-Emx ${ }^{\text {Cre }}$ at E17.5; level of Notch signaling is not affected. Immunostaining for phosphorylated Smad1/5/8, downstream effectors of BMP signaling, at E17.5 indicates strong activity in the VZ in Wt (a) and in basally dispersed cells (arrowhead) in Sip1fl/fl-Emx ${ }^{\text {Cre }}$ cortices (b). Extent of Notch signaling was detected by staining for the intracellular domain of activated Notch (NICD); shown here is ventricular localization of the same at E16.5 in both Wt (c) and Sip1fl/fl-Emx ${ }^{\mathrm{Cre}}$ (d). No changes in NICD expression was observed at E13.5 (e,f). Levels of NICD in cortical protein extracts from E16.5 was checked by Western blot (g). No differences were seen between Wt and Sip1fl/fl-Emx ${ }^{\text {Cre }}$. Expression of JNKs in cortical tissue was also not affected, as detected here on a Western blot using a pan- JNK antibody $(\mathrm{g})$.

(Gross et al., 1996; Nakashima et al., 2001). While we detected strong BMP activity in VZ cells in wildtype cortices at E17.5, in Sip1 fl/fl-Emx ${ }^{\mathrm{Cre}}$ these were dispersed away from the VZ (Fig17a,b), a distribution that is clearly reminiscent of the ectopically dividing $\mathrm{Ki} 67+/ \mathrm{PCNA}+/ \mathrm{Blbp}+$ cells that we observed in both conditional mutants at this stage (Fig5c-h). This in turn suggests that the excess of ectopically dividing cells seen in the 
mutant cingulate cortex at E17.5 are indeed astrocytic precursors, and that BMP signaling drives these cells towards differentiating into GFAP+ astrocytes. Also interesting to note was the high medial to low lateral gradient of BMP activity at the VZ, considering that there is a BMP signaling centre at the cortical hem close to the medial cortex. This gradient however does not seem to have changed in mutant cortices. Although dispersed pSmad1/5/8+ cells can definitely be seen in mutant cortices at this stage, it has not yet been determined if there are more cells showing BMP activity. At E16.5, we did not detect any increase in $\mathrm{pSmad} 1 / 5 / 8$ staining in the mutant.

\subsubsection{Notch signaling is not affected in Sip1 depleted cortices}

One of the pleiotropic functions of Notch receptor/ Delta ligand mediated signaling is to promote astrocytic differentiation; during corticogenesis this happens during late stages (Chambers et al., 2001; Gaiano et al., 2000; Ge et al., 2002; Grandbarbe et al., 2003; Louvi and Artavanis-Tsakonas, 2006; Tanigaki et al., 2001). On being activated, the intracellular domain of Notch (NICD) gets cleaved, translocates to the nucleus, and activates transcription of target genes (Louvi and Artavanis-Tsakonas, 2006). We immunostained E13.5-E16.5 wildtype and mutant cortices for NICD, a marker of Notch activity. As expected, at E16.5 most of the activity was observed at the VZ; however, based on the pattern and intensity of staining, we did not find any differences between wildtype and Sip1fl/fl-Emx ${ }^{\text {Cre }}$ cortices (Fig17c,d). In order to further confirm that there is indeed no enhancement of Notch activity in mutant cortices, we compared the levels of NICD in both tissues at E16.5 by Western blot (Fig17g) Again, we found no differences. At E13.5 NICD signal was seen all over the cortex in both wildtype and Sip1fl/fl-Emx ${ }^{\mathrm{Cre}}(\mathrm{Fig} 17 \mathrm{e}, \mathrm{f})$ while at E14.5, we could detect only very weak activity; there do not seem to be any significant differences. At these stages, the staining was detected around rather than inside the nucleus. Although in some sections, NICD levels did seem to be higher in mutant cortices at E13.5, this observation has to be confirmed by Western blotting, to exclude common staining artifacts. Next, we checked the expression of JNKs (c-Jun N- terminal kinases) that act downstream of Wnt ligand- Fzd receptor interaction in a non- canonical signaling pathway. We did not detect any differences in the expression levels of JNK (Fig17g), but could not estimate the levels of phosphorylated, and hence activated, JNK. 


\subsubsection{In vitro experiments fail to demonstrate the ability of early cortical progenitors to adopt later cell fate in a Sip1 deficient microenvironment}

\subsubsection{Differentiation of younger progenitors on older cortical slices}

Based on our result that young cortical progenitors can respond to deletion of Sip1 in the cortical plate and switch fate to later cell types, we proposed a hypothesis. We speculated that the extracellular signals received by a progenitor should be very critical to determining its fate. In young wildtype embryos, Sip1, being primarily a transcriptional repressor, partially represses the expression of a secreted factor in the cells of the cortical plate; this in turn ensures differentiation of early precursors into deep layer neurons (Fig18a). In older embryos, with increase in the number of cells in the cortical plate, and hence, increased production of this secreted molecule, the concentration of the latter crosses a threshold value and induces VZ/SVZ cells to differentiate into upper layer neurons instead (Fig18b). When Sip1 is mutated, the repression of this factor is inhibited, as a consequence of which, its levels reach the threshold earlier, and the microenvironment in the early cortex becomes more conducive to upper layer generation (Fig18c). Hence, if early precursors were to be plated on an older cortical slice, they should differentiate into upper instead of deep layer neurons. In order to investigate the plausibility of such a mechanism, we performed Slice overlay assays.

In the first set of experiments, we isolated cortices from GFP expressing transgenic mice and dissociated them into single cells. These were then overlaid on E13.5 and E15.5 wildtype slices and cultured for 2-4div. The slices were then fixed and immunostained for Satb2 (to label upper layer neurons) and Ctip2 (to label deep layer neurons). Under the conditions that were used for culturing, two kinds of GFP+ cells were observed- those that formed neurospheres all over the cortex, and those that integrated into the slices and differentiated into neurons (Fig18d-g). We calculated the proportion of GFP+Satb2+ and GFP+Ctip2+Satb2- cells over the total number of GFP+ cells. Since it was recently reported that cells expressing both Ctip2 and Satb2 at any stage of development eventually become Satb2+Ctip2- upper layer neurons (Britanova et al., 2008), we decided to consider all GFP+Ctip2+Satb2+ cells as those destined to become GFP+Satb2+. From two independent experiments, we found that while the proportion of Ctip $2+\mathrm{GFP}+$ cells remains the same on E13.5 versus E15.5 slices, the proportion of GFP+Satb2+ cells is 2-3 times 

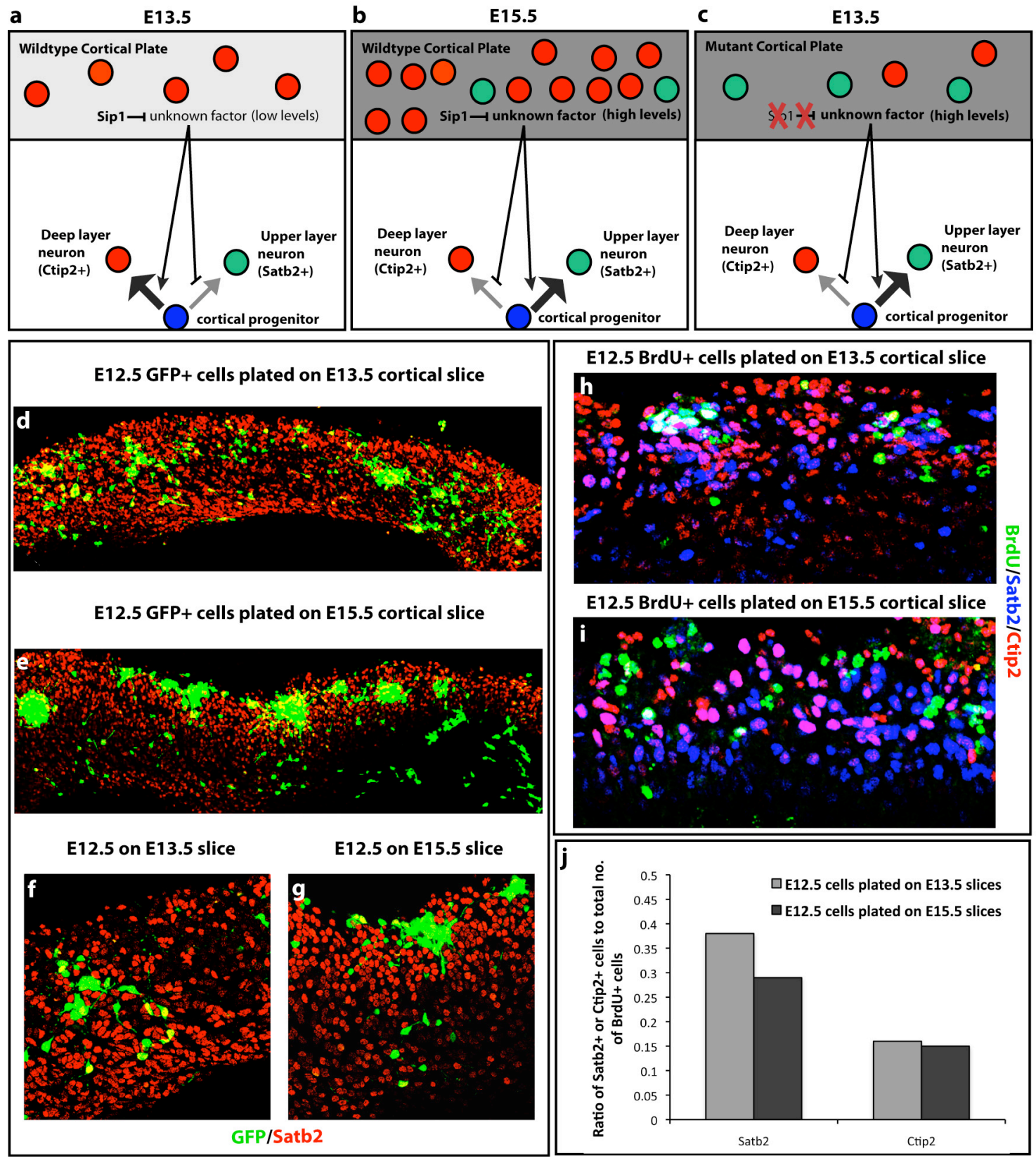

Fig18. Neuronal differentiation of young cortical precursors on older slices in vitro. The model shown in a-c proposes the existence of an unknown factor whose expression is partially repressed by Sip1 in the cortical plate neurons. At early stages (E13.5), fewer CP neurons result in below threshold concentration of this factor leading to generation of deep layer neurons by VZ progenitors (a). At later stages (E15.5), increased number of neurons result in concentrations above the threshold, leading to generation of upper layer neurons (b). In Sip1 mutants, absence of Sip1 releases the repression on the production of this factor, leading to it reaching threshold levels earlier than normal (E13.5), which in turn, would lead to premature generation of upper layer neurons (c). GFP+ wildtype E12.5 cortical cells plated on E13.5 (d,f) and E15.5 $(e, g)$ slices and cultured for $3 \mathrm{div}$ can be seen to integrate into the slices, divide to form small clusters of cells, 
extend processes, and also form neurospheres throughout the medio- lateral axis. Some of them express Satb2 (d,e). Shown here are images procured at 20X (d,e) and 40X (f,g). Dividing cells at E12.5 labeled with a half hour BrdU pulse plated on E13.5 (h) and E15.5 (i) cortical slices were immunostained for Satb2 and Ctip2 after 2div. Quantification from three independent experiments (two with GFP+ E12.5 cells and one with BrdU+ E12.5 cells (j)) indicates reduced ability of E12.5 precursors to differentiate into Satb2+ cells on E15.5 slices in comparison with E13.5 slices. The proportion of cells differentiating into Ctip2+ layer 5 neurons, however, does not change with the age of the cortical slice $(j)$.

lesser on E15.5 slices than on E13.5 slices. This is an intriguing observation. It is possible that of the cells that are being plated, some are already committed towards either a deep layer fate or an upper layer fate, and irrespective of the age of the cortical slice, they differentiate into Ctip2+ layer 5 neurons or Satb2+ upper layer neurons. It seemed to us that the cells whose fate was being mapped were already committed to one of the two lineages. Since at E12.5 there is already a sizeable population of committed precursors as well as young neurons in the neocortex, our experimental setup could be misleading. To overcome this problem, and to ensure that we are tracing the fate of only dividing cells, we designed a second approach.

In this second set of experiments, a subpopulation of dividing cells at E12.5 were labeled by intraperitoneal BrdU injections half an hour before dissociating and plating on E13.5/E15.5 cortical slices. After 4div, they were stained for BrdU, Satb2 and Ctip2 (Fig18h,i). Since neither Satb2 nor Ctip2 is expressed in proliferating cells, we were assured that all BrdU+Satb2+/BrdU+Ctip2+ cells differentiated after plating on the slices. We determined the relative proportions of BrdU + cells that became either Satb2+ or Ctip2+. Once again, while the proportion of Ctip2 differentiation did not vary between E13.5 and E15.5 conditions, the percentage of BrdU+ cells that became Satb2+ was lesser on E15.5 slices than on E13.5 slices (Fig18j). It is quite likely that early UL progenitors are unable to respond to the differentiation cues in a late stage cortex. Altogether, it seems that at least in our culture system, extracellular conditions cannot influence the fate of early (E12.5) cortical progenitors. Interestingly, we also noted that the percentage of plated cells that become Satb2+ is higher if only dividing cells are considered instead of all cortical cells. On the other hand, the percentage of plated cells that become Ctip2+ is lower. This could imply that a considerable amount of cells at E12.5 (GFP+), that were either in their last cell cycle or just exit the cell cycle before being overlaid on E13.5/E15.5 slices, were already committed towards a layer 5 lineage. 


\subsubsection{Differentiation of younger progenitors on older wildtype versus mutant cortical slices}

We have shown that in Sip1fl/fl-Nex ${ }^{\mathrm{Cre}}$, the onset of gliogenesis is shifted by at least one day, and the proliferation of astrocytic precursors is enhanced at E17.5. These observations raise the possibility that absence of Sip1- mediated feedback signaling from the cortical plate to the VZ/SVZ coerce cortical progenitors to adopt a gliogenic fate. In other words, a gliogenic cue exists in the Sip1- deficient cortex (Fig19a,b). Since it has been shown that young precursors are responsive to cues that can trigger them to differentiate towards an older fate, we hypothesized that if early precursors were to be plated on an E16.5/E17.5 mutant slice, these cells would differentiate into astrocytes more frequently than when plated on wildtype slices (Fig19a,b). Therefore, in the next set of Slice overlay assays, E13.5-E14.5 GFP+ cells were overlaid on E16.5-E18.5 wildtype and mutant cortical slices and cultured for 6-7div. We used both Sip1fl/fl-Emx ${ }^{\mathrm{Cre}}$ and Sip1fl/fl-Nex ${ }^{\mathrm{Cre}}$ slices for this experiment.

We first did a pilot experiment to prove that in our assay system, number of E13.5 GFP+ cells differentiating into GFAP+ astrocytes increases progressively with the number of days in culture, when plated on E16.5 wildtype slices. The first instances of GFP+ cells differentiating into GFAP+ astrocytes are detected at 7div. Thereafter, astrocytic differentiation increases steadily (Fig19c).

However, when we compared wildtype and mutant cultures for the proportion of cells differentiating into astrocytes, we detected, based on preliminary results, a higher propensity of E13.5 cortical cells to differentiate into astrocytes on E17.5 wildtype slices $(5.3 \%)$ than on Sip1fl/fl-Nex ${ }^{\text {Cre }}$ slices (1.4\%) (Fig19d-f) after 6div. Once again, to ensure that we trace the fate of only those E13.5 cells that were dividing at the time of overlay, a cohort of progenitors were labeled with BrdU by intraperitoneal injections half an hour before dissociating and plating. After 7div, slices were stained for GFAP and BrdU. To overcome the technical difficulties in colocalising BrdU and GFAP signals, we tried to image $z$ - stacks and reconstruct $3 \mathrm{D}$ projections to assay for the same. Preliminary analysis indicated no differences in the proportion of BrdU+GFAP + cells between wildtype and mutant slices. 

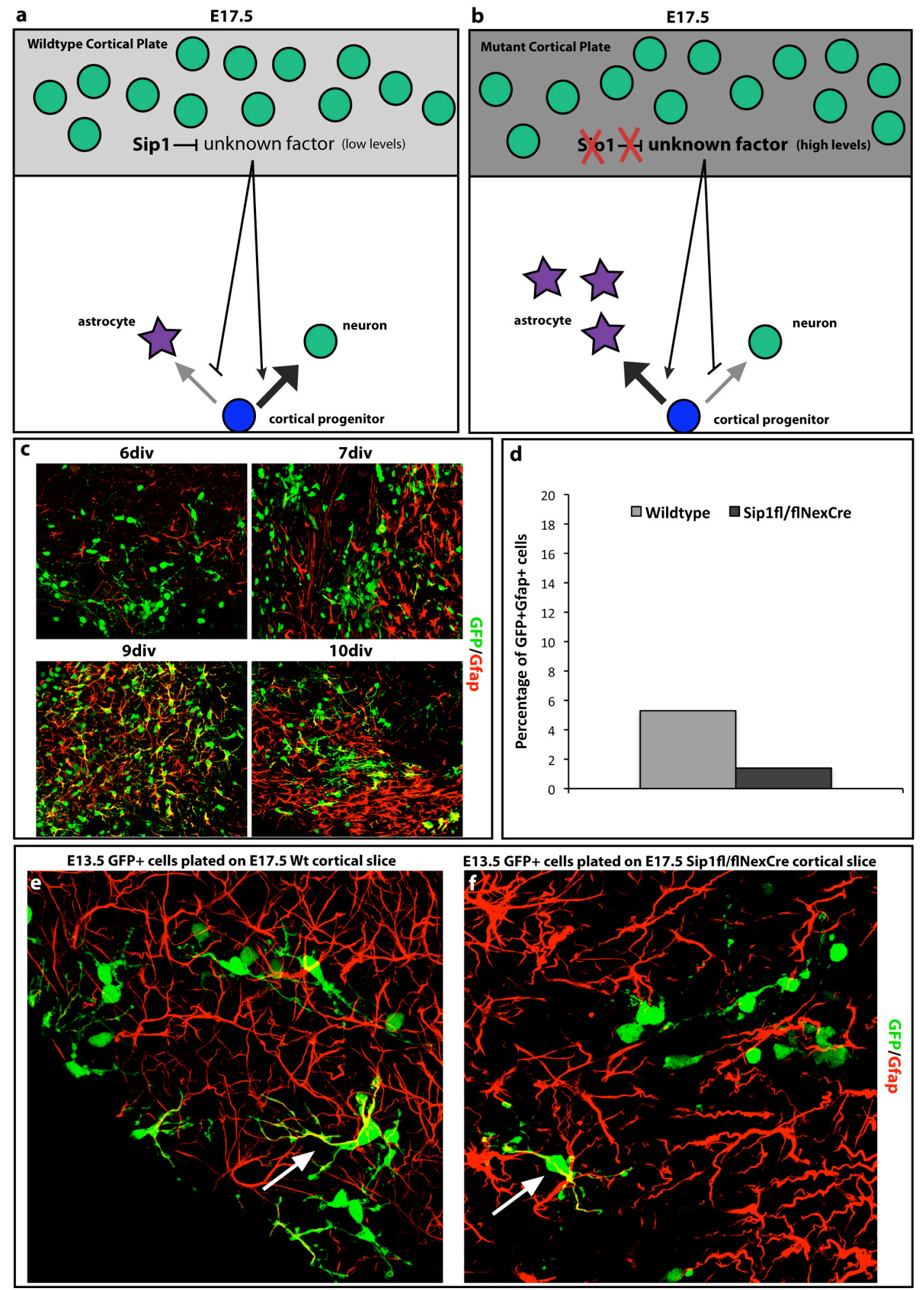

Fig19. Gliogenic differentiation of early precursors on older wildtype and mutant cortical slices in vitro. The model shown in a-b proposes the existence of an unknown factor whose expression is partially repressed by Sip1 in the cortical plate neurons during late corticogenesis (E17.5). In wildtype cortices, low levels of this factor control the propensity of progenitors to generate astrocytes (a). In mutant slices, absence of Sip1 releases the repression of this factor leading to high concentrations of the latter, which in turn induces 
premature and enhanced astrocytogenesis (b). To determine the propensity of GFP+ E13.5 cortical cells to differentiate into GFAP+ astrocytes in vitro, they were plated on E16.5 slices and cultured for 5-10 div (c). The first GFP+ astrocytes could be seen after 7div, and their numbers increased progressively with increasing number of days in culture. Shown here are stainings at 6, 7, 9 and 10div (c). GFP+ E13.5 cells overlaid on E17.5 wt (e) and Sip1fl/fl-Nex ${ }^{\mathrm{Cre}}$ (f) slices and cultured for 6div were immnostained for GFAP, and the presence of GFP + GFAP + cells was analysed. Preliminary results suggested higher percentage of GFP + cells becoming GFAP+ on wt than on mutant slices (d).

Furthermore, when we plated BrdU labeled E14.5 precursors on E18.5 slices (astrocytic period in corticogenesis), and calculated the proportion of BrdU+ cells differentiating into either Satb2+, or Ctip2+, or Satb2-Ctip2- (presumably astrocytic) cells, we found no differences in either of these values between wildtype and Sip1fl/fl-Emx ${ }^{\mathrm{Cre}}$ slices.

\subsubsection{Deletion of Sfrp1 in Sip1 conditional knockouts does not rescue the neocortical defects seen in these mutants}

Previous work done in the lab involved an extensive study of the expression of the Wnt antagonist Sfrp1 in wildtype and Sip1 mutant cortices (Miquelajauregui et al., 2007). It was found that not only is it expressed in a pattern complementary to that of Sip1 in wildtype cortices at embryonic to early postnatal stages, but that it is also dramatically upregulated all over the cortex at E17.5 in the absence of Sip1 (Miquelajauregui et al., 2007). Since this phenotype coincided with the increased proliferation of astrocytic precursors in Sip1 mutants, we hypothesised that Sfrp1, a secreted molecule, signals back to $\mathrm{VZ} / \mathrm{SVZ}$ progenitors and triggers either proliferation of existing glial precursors or induces a cell fate switch towards an astrocytic lineage. We performed several experiments to confirm this hypothesis. First of all, we bred both Sip1 conditional mutants with Sfrp1 total knockouts to obtain double mutants that lacked both Sip1 as well as a functional copy of Sfrp1 allele. This was followed by analysis of deep layer populations, and postnatal astrocytogenesis and proliferation. The rationale behind this approach was that in Sip1 mutants, in the absence of Sfrp1, no upregulation would be possible and hence all the phenotypic effects mediated by it would be rescued. However, we detected no such rescue. We began our analysis by confirming deletion of Sfrp1 in these mutants. In the absence of a good antibody against Sfrp1, we performed in situ hybridisation to confirm deletion of Sfrp1 mRNA in the double mutants. As shown, Sfrp1 transcripts seem to have reduced in comparison to wildtype and Sip1fl/fl-Emx ${ }^{\mathrm{Cre}}$ (Fig20a-c). We then immunostained P8 


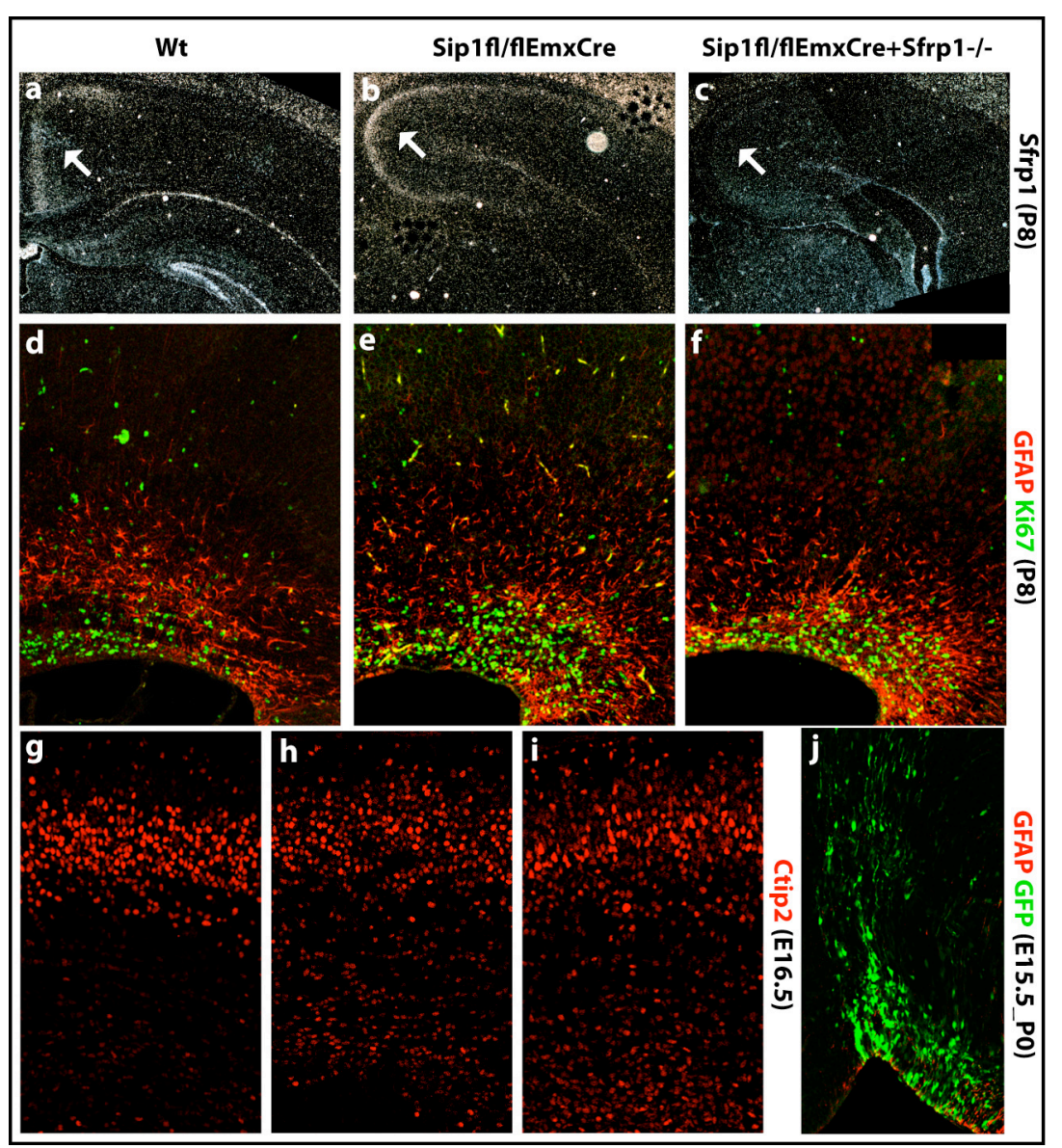

Fig20. Ablation of Sfrp1 in Sip1 conditional mutants does not rescue the mutant phenotype. A probe targeted against the deleted exon of Sfrp1 gene was used to detect the level of expression in Wt, Sip1fl/flEmx ${ }^{\mathrm{Cre}}$, and Sip1fl/fl-Emx ${ }^{\mathrm{Cre}}$ Sfrp1-/- , by in situ hybridization at P8 (a-c). The reduction in Sfrp1 transcript levels can be seen in the double mutant. Immunostaining for GFAP and Ki67 at P8 clearly shows no rescue of Sip1 mutant phenotype in the double mutants (d-f). The reduction of Ctip2+ cells at E16.5 in Sip1fl/fl$\mathrm{Emx}{ }^{\mathrm{Cre}}$ remains in the double mutant as well (g-i). Overexpression of Sfrp1 (alongwith GFP reporter) in the cingulate cortex by in utero electroporation at E15.5, followed by analysis at P0 shows no colocalisation between GFP and GFAP (j), and hence, possibly no influence of Sfrp1 on cortical gliogenesis.

cortices for GFAP and Ki67. As expected, there was an increased expression of both in Sip1fl/fl-Emx ${ }^{\mathrm{Cre}}$ especially in the region of the cingulate cortex. This enhanced expression was observed in the double mutants as well, implying clearly that deletion of Sfrp1 could not rescue the gliogenic phenotype of Sip1 mutants (Fig20d-f). Although the upregulation of Sfrp1 in the neocortex at early embryonic stages was not obvious, we nevertheless checked for expression of deep layer markers in the double mutants at E16.5. Again, the reduction in deep layer neuronal populations seemed as strong as in Sip1 mutants (Fig20g- 
i). Finally, to test possible cell autonomous as well as non- cell autonomous effects of overexpressing Sfrp1 (since Sfrp1 is a secreted molecule) in the cingulate cortex, especially in context of gliogenesis, we adopted an in utero approach. Here, in collaboration with Dr. Federico Calegari (Max Planck Institute of Cell Biology and Genetics, Dresden), Sfrp1 overexpressing constructs were electroporated into E15.5 forebrain, which were then analysed at P0 for GFAP expression. The constructs encode Sfrp1 under the CMV promoter, and also contain GFP coding sequence under the SV40 promoter. We found GFP expression close to the ventricle, in migrating cells as well as some cells in the cortical plate (Fig20j). However, we found no differences in GFAP expression between the electroporated and control hemispheres, and also no colocalisation between GFP and GFAP (Fig20j). This observation suggests that Sfrp1 might not influence cortical astrocytogenesis, neither within the same cell nor on neighbouring cells. However, it is also likely that P0 is too early a stage to check GFAP expression; an alternative would be to use an earlier expressed marker like Olig2. 


\section{DISCUSSION}

In an attempt to explore the puzzling and complex dynamics of cortex development, we set out to investigate the functions of a transcriptional repressor expressed at very high levels in the developing neocortex almost exclusively in its differentiating field. From a subtractive hybridisation based screening, Sip1 was identified as a gene differentially expressed between early and late stages of mouse corticogenesis, and hence selected for further studies. Since Sip1 deficient mice have been shown to die at E9.5 with major anomalies in neurulation and neural crest migration, we opted to generate and analyse cortex- specific conditional knockouts. We have shown that in the neocortex, strong expression of Sip1 is confined predominantly to postmitotic cells, starting at E12.5 until early postnatal stages. Most, though not all, upper and deep layer neurons express Sip1. At P8, Sip1 transcripts were detected primarily in the postmitotic cingulate and medial cortex, while Sip1 protein could be detected in very few cells scattered throughout the neocortex. We have also shown that E17.5 onwards, the expression of Sip1 in the cingulate cortex is stronger in layers 5-6 than in layers 2-4.

\subsection{Sip1 controls sequential cell fate switch in cortical progenitors during the course of embryonic development}

On the basis of our analysis of two different cortex- specific conditional knockouts of Sip1, we have been able to demonstrate that this transcriptional repressor plays a key role in regulating the timing of cell fate switch in the neocortex by controlling the activity of certain signaling pathways.

Sip1 deficient neocortex is thinner, possibly due to fewer neurons, accompanied by reduced layers 5 and 6 and a concomitant expansion of layers 2-4 at prenatal stages of development. Possible reasons would include alterations in proliferation, cell cycle kinetics, apoptosis, neuronal fate specification and/or differentiation.

Our analyses have shown that specific deletion of Sip1 in the postmitotic compartment of the neocortex leads to premature specification of cortical progenitors towards an upper layer lineage at early stages, and a glial lineage at later stages of corticogenesis (Fig21). 
While the dynamics of generation of the early born layer 6 neurons is not affected in Sip1 conditional mutants, Ctip2+ layer 5 neurons, Foxp1+ layer 3-5 neurons, and Satb2+ and Brn2+ layer 2-4 neurons are all generated prematurely (Fig8). Their peak of production is shifted temporally by at least one day. It is important to note that for all of these cell types, irrespective of their usual time of generation, in Sip1 mutants the peak of production is shifted to earlier stages (E12.5-E13.5). On the other hand, we detected no alteration in either the extent of proliferation or the rate of mitotic exit at these stages. In fact, expression of $\mathrm{Hu}$ (a marker of young postmitotic neurons) at E12.5 and E13.5 indicates no changes in either the thickness or the cell density of the mutant cortical plate (Fig6). This implies, together with the results of Tbr1 birthdating, that the onset of neurogenesis in Sip1 mutants is not altered. Furthermore, we have also determined the function of Sip1 in controlling the timing and extent of astrocyte production in the neocortex. Our pulse chase experiments prove increased and earlier specification of both Olig2+ as well as GFAP + glial cells in the mutants (Fig9). Altogether, we believe that the onset of neurogenesis in the cortex is not affected by Sip1 deletion; however, once the process commences, Sip1 plays a major role in the temporal control of the sequential generation of different cell types, irrespective of the number of each type generated.

From our extensive analysis of proliferation and cell cycle kinetics in Sip1 mutants, we can conclude that Sip1 has no influence on mitotic divisions of early cortical progenitors. However, Sip1 does induce expansion of the pool of glial progenitors (expressing Blbp/ Olig2) at E17.5 in a non- cell autonomous manner (Fig7).

Apoptosis is not responsible for the reduction of deep layer neurons in Sip1 mutants. Although it has been shown before that apoptotic cell death increases in layer 6 in Sip1 mutant cortices at early postnatal stages (Doctoral Thesis, Miquelajauregui A, Mar 2006, University of Goettingen, unpublished data) we did not detect any such effect during early embryonic development, when the reduction in deep layers is already obvious. This suggests that apoptotic cell death is not the primary reason behind the appearance of fewer layer 5/6 neurons in mutant cortices.

The fact that GFAP+ astrocytes are detected in mutant cortices not before their usual time of appearance, albeit at higher numbers, indicates that the function of Sip1 might be limited to influencing the commitment of progenitors towards a certain lineage and inducing them to expand their pool size, but that the final differentiation takes place at its 

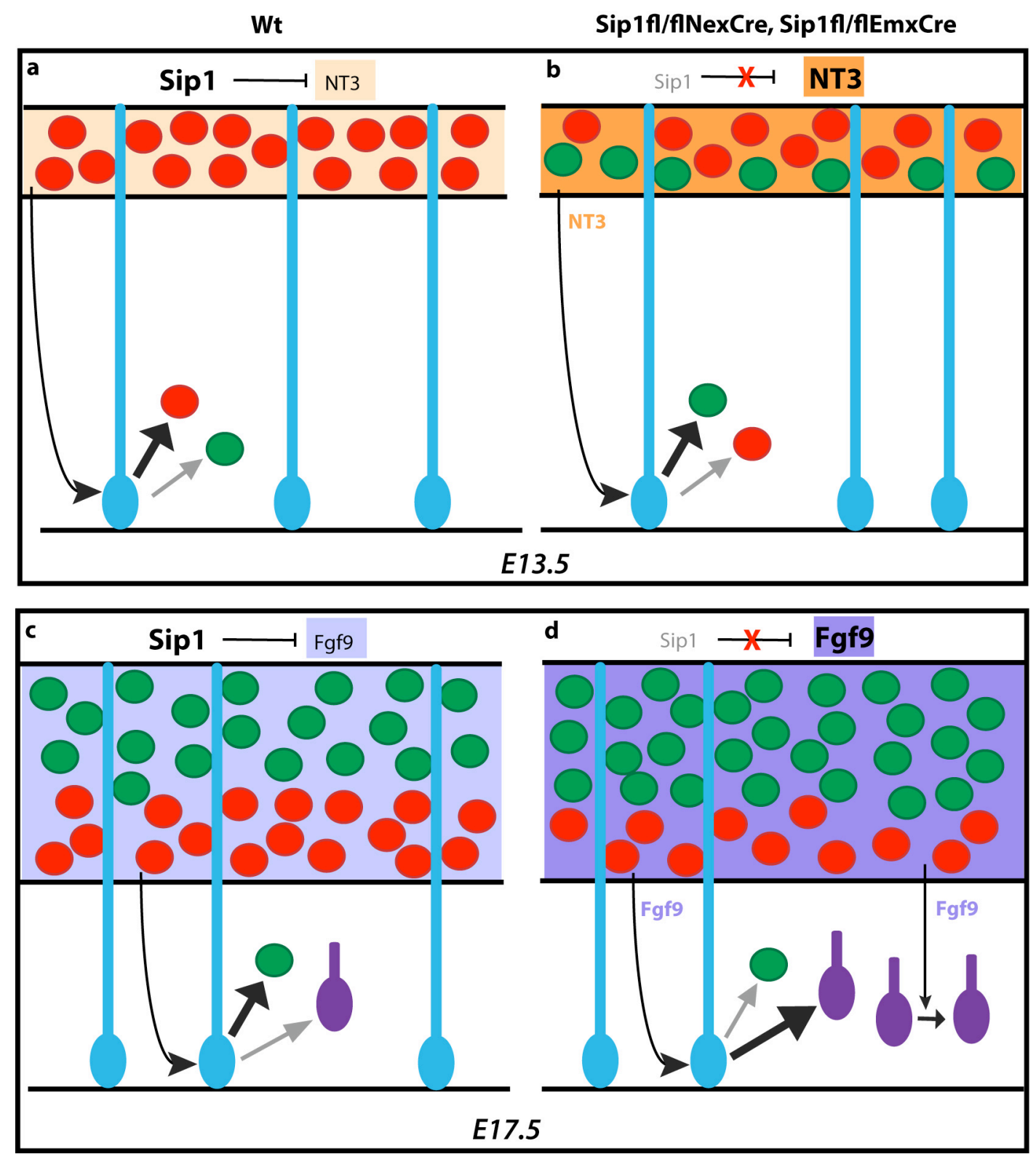

Deep layer neuron

Upper layer neuron

\begin{tabular}{l|l}
$\begin{array}{l}\text { Radial glial } \\
\text { cell/neuronal } \\
\text { progenitor }\end{array}$ & $\begin{array}{l}\text { Radial glial } \\
\text { cell/glial } \\
\text { progenitor }\end{array}$
\end{tabular}

Fig21. A model proposing Sip1- mediated non- cell autonomous control of cortical progenitor fate. At early stages of corticogenesis (E13.5), Sip1, expressed strongly by cortical plate neurons, represses NT3 expression, which in turn ensures generation of deep layer rather than upper layer neurons (a). In Sip1 conditional mutants, lack of Sip1 leads to premature overexpression of NT3, which signals back to VZ progenitors to induce a fate switch from DL to UL neuron production (b). At later stages (E17.5), Sip1 represses Fgf9 expression in $\mathrm{CP}$ neurons, and feedback signaling from $\mathrm{CP}$ to $\mathrm{VZ}$ ensures a precisely regulated end of neurogenesis and onset of gliogenesis (c). In Sip1 conditional mutants, absence of Sip1 in the $\mathrm{CP}$ leads to precocious upregulation of Fgf9, which signals back to $\mathrm{VZ}$ precursors to firstly, induce 
premature neurogenic to gliogenic fate switch, and secondly, to promote proliferation of progenitors committed to an astrocytic lineage (d).

own designated time. However, in case of Brn2+ upper layer neurons, which are indeed detected precociously in the mutant cortex, even the differentiation of committed progenitors seems to have shifted to at least one day earlier. This, however, may not be completely true, since Brn2 is known to be expressed in migrating neurons as well, before they fully differentiate into mature upper layer neurons. Since our claim is based mostly on BrdU pulse- chase experiments, there could be an alternative explanation to our observations. The possibility that upper layer neurons are born in their usual temporal sequence but that it is the deep layer neurons that undergo a postmitotic cell fate shift to an upper layer neuron by early postnatal stages, cannot be precluded. On the other hand, if we were to apply the same logic to the neuron to glial fate switch at later stages, the likelihood of a neuron transdifferentiating into an astrocyte seems very low. Also, using the Fgf9 coated beads on slice cultures, we have shown that the increased number of Olig2 + cells is confined to the region close to the VZ (Fig15). This implies that Fgf9 expression in the cortical plate signals back to the VZ/SVZ and induces proliferation of glial precursors. Moreover, it is highly likely that Sip1 acts via the same conceptual mechanism at both early and late stages, though the intermediate signaling pathways might be different. Therefore, we strongly suspect Sip1 to non- cell autonomously influence progenitor commitment rather than be involved in maintenance of the differentiated state of a neuron.

So far, several instances of premature astrocyte production in the cortex have been reported. These include, 1. Exogenous application of growth factors such as LIF, CNTF, CT-1, Fgf2, CNTF and BMPs to neural stem cell cultures; 2. lack of function of neurogenic bHLH genes such as Ngn2,Mash1 and Ngn1 (Nieto et al., 2001; Sun et al., 2001); 3. Overexpression of EGF receptor (Burrows et al., 1997; Sun et al., 2005); 4. Genetic ablation of certain growth factors, receptors, signaling molecules and transcription factors. Some examples include, neuregulin receptors ErbB2 and ErbB4 (Sardi et al., 2006; Schmid et al., 2003), SHP-2, a growth factor regulated phosphatase known to modulate MEK-ERK and gp130-JAK-STAT pathways (Miller and Gauthier, 2007) and N-CoR (Hermanson et al., 2002) (other reports reviewed in (Miller and Gauthier, 2007). Our studies report for the first time, precocious astrocytogenesis as a consequence of deletion 
of a postmitotic transcription factor leading to gliogenic feedback signaling from the cortical plate to the VZ/SVZ.

The only instances of premature cortical neurogenesis that have been reported so far involve a shift in the peak of the entire neurogenesis and not just of some cell types, as we have shown in our conditional mutants. Examples of the former include, knockout of Id4 (Yun et al., 2004) and several Notch pathway related mutants (reviewed in (Yoon and Gaiano, 2005).

\subsection{Selective role of Sip1 in progenitor self- renewal in the cortex}

The very first cortical precursors determined to generate astrocytes appear at E15.5/E16.5 in wildtype mice. Lack of Sip1 in the cortical plate results in firstly, increased probability of gliogenic specification, and secondly, an active expansion of the pool of gliogenic progenitors. We have shown that most of the astrocytic precursors in Sip1 conditional mutants are located abventricularly, and express BLBP, Olig2 and Pax6, while a few of them express Tbr2 (Fig7). Besides this population, we also detected an expansion in the population of Tbr2 expressing non- dividing (Ki67-PCNA-) cells at the SVZ in the cingulate cortex in Sip1fl/flNex ${ }^{\mathrm{Cre}}$ at E17.5 (Fig7). This is an intriguing observation since on one hand, our data indicate a premature end of neurogenesis in Sip1 mutants, while on the other hand Tbr2+ cells have never been shown to generate glial cells (Kowalczyk et al., 2009; Noctor et al., 2004). It would be interesting to characterise and trace the fate of these cells in the future.

Surprisingly, although it seems that absence of Sip1 leads to a premature commitment of early progenitors towards an upper layer lineage (layers 2-4, shown on the basis of 3 molecular markers) we did not detect any increase in Tbr2 expression at early stages of development until E15.5. Contradictory to our observations at E17.5, the number of Tbr2+ cells in the IZ, and possibly in the SVZ as well, is reduced in Sip1 conditional knockouts at E15.5, presumably marking the premature end to neurogenesis. Tbr2 was shown to be essential for the production of Satb2+, Brn2+, Svet1+ and Cux2+ upper layer neurons, though it must be pointed out that in Tbr2 conditional knockouts, upper layers do not fail to form. They only lack expression of common upper layer markers (Arnold et al., 2008). Nevertheless, Tbr2 is commonly considered as a marker of upper layer progenitors. Since 
there are no reported molecular markers to differentiate between precursors committed to producing layer 6 neurons versus those committed to generate layer 5 neurons, it would be difficult to detect an increase in layer 5 progenitors in Sip1 mutant cortices.

We also show that the increased proliferation that commences at E17.5 in the absence of Sip, continues atleast until P8 (Fig10). While some of these dividing cells express Olig2 and are hence, gliogenic, there are several that express GFAP, or neither of the two. Those that express GFAP could be either astrocytic or multipotent, since GFAP is expressed in postnatal and adult neural stem cells of the cortical SVZ, which can potentially generate both neurons and glia (Doetsch et al., 1999; Imura et al., 2003; Laywell et al., 2000). Those that express neither Olig2 nor GFAP are likely to be a type of non- committed progenitors.

\subsection{Role of Sip1 in establishing the morphology of the neocortex}

\subsubsection{Deletion of Sip1 hampers migration of late born cortical neurons}

We have seen that a delay in radial migration of young neurons born at and after E15.5 is seen in both Sip1 conditional mutants, despite the fact that while in Sip1fl/fl-Emx ${ }^{\mathrm{Cre}}$, Reelin expression is reduced and might partially explain the phenotype, in Sip1fl/fl-Nex ${ }^{\mathrm{Cre}}$ it remains unaffected (Fig11). It has been shown that overexpression of Reelin in the ventricular zone cells of reeler mice can lead to a rescue of the inverted cortical layers in these mutants, implying that the physical location of the source of Reelin is not so significant as long as there is a supply within the cortex (Magdaleno et al., 2002). The dispersion of Reelin- secreting cells at E14.5, before the onset of the phenotype, therefore, might not really add to the subsequent hampered migration of neurons. Moreover, the morphology of radial glial processes, often used by migrating neurons as a scaffold, is unaffected in Sip1 conditional mutants (Fig12). Altogether, this implies that the general migration delay in these mutants is either due to a deficiency in effectors downstream of Reelin or due to a defect in alternative pathways like the $\mathrm{p} 35 / \mathrm{Cdk} 5$ mediated signaling.

In this context, we made an interesting preliminary finding. We found that one of the putative Sip1 targets in the cortex, Itga6 (Integrin alpha 6, a cell- matrix adhesion molecule), is downregulated at E14.5 and upregulated at E16.5 in Sip1fl/fl-Emx ${ }^{\mathrm{Cre}}$, as determined by semi- quantitative PCR (Fig13). Though not much work has been published 
on putative functions of Itga6 in cortical lamination/migration, there are indications that it might indeed be involved in the same (Georges-Labouesse et al., 1998). In any case, dynamic changes in adhesion properties between cells and the extracellular matrix are expected to influence the rate of migration of young neurons away from the germinal zones (Anton et al., 1999; Schmid et al., 2004). We believe that changes in the level of Itga6 might influence the former, and therefore be partly responsible for the hampered migration of late born upper layer neurons.

Interestingly, the migration of earlier born neurons seems unaltered, if not faster. We have shown that in Sip1 conditional mutants, cell born at E12.5-E13.5 mostly occupy upper cortical layers (Fig11p,q). Coupled to the fact that these cells express upper layer neuronal markers, this particular phenotype seems to be a consequence of premature shift in cell fate specification of cortical progenitors rather than abnormalities in migration of young neurons.

\subsubsection{Disorganised stratification of cortical layers is due to both premature production of layer 2-5 neurons as well as delayed migration of late born UL neurons}

Compaction of the six different layers of the neocortex is clearly disrupted in both Sip1 conditional knockouts. Defective neurogenesis could be largely responsible for this phenotype. In wildtype embryos, cells born at E12.5/E13.5 are distributed radially throughout the neocortex, but localised mostly to layers 5-6. In Sip1 mutants, however, most of them are located in the upper layers 2-4, possibly due to the premature specification of these neurons. Accompanied by the fact that neurons born at and after E15.5 are fewer and seem to migrate slower in the mutants than in the wildtype, it is not surprising that the final lamination of the neocortex is distorted. In other words, the hampered migration at later stages and cell fate shift at earlier stages, leads to a loss of compaction and hence, dispersion, of cortical layers later.

\subsubsection{Thinner cortex and absence of corpus callosum in Sip1 conditional mutants}

The reduction in thickness of the Sip1 mutant cortex is intriguing. Considering that in these mutants, early proliferation is not changed and that late proliferation is actually increased, without much difference in the level of apoptosis, it is indeed surprising to find an overall 
thinning of the cortex. However, we did observe a premature end to neurogenesis despite the fact that the onset of neurogenesis does not seem to be shifted, based on birthdating of Tbr1+ layer 6 cells, and expression of neuronal marker Hu at E12.5. This in turn implies that the overall duration of neurogenesis is reduced in Sip1 mutants without any changes in the rate of proliferation, mitotic exit, or cell cycle length (Data from collaboratorsSeuntjens E, Huylebroeck D), possibly leading to the generation of fewer neurons, and hence a thinner cortex. Also, the extent of apoptosis, though seen to some degree in deep layers at early postnatal stages (Doctoral Thesis, Miquelajauregui A, Mar 2006, University of Goettingen, unpublished data) does not seem to be high enough to result in a thinner cortex. Moreover, although the shift in neuronogenesis of layers 2-5 is followed by premature gliogenesis, the increase in astrocyte production is apparently not sufficient to make up for the overall size reduction. The function of the excess of astrocytes in the mutant cortex is yet to be determined.

It is interesting to note that in contrast to the rest of the neocortex, expression of Sip1 in the cingulate cortex is restricted to deep layer neurons at E17.5, and that absence of Sip1 leads to agenesis of the corpus callosum and formation of a Probst bundle. It has been shown that some of the earliest born cells of the cingulate cortex extend pioneering axons to initiate corpus callosum formation (Koester and O'Leary, 1994; Rash and Richards, 2001). Together, this suggests that in the cingulate cortex, Sip1 might have a function different from its role in the rest of the dorsal cortex. Moreover, It has been reported previously that neither the integrity of the cortical hem nor BMP signaling at the dorsal telencephalic midline is affected in Sip1 conditional mutants (Doctoral Thesis, Miquelajauregui A, Mar 2006, University of Goettingen, unpublished data).

Besides the corpus callosum, a second thinner bundle of fibres connecting the two cerebral hemispheres is the anterior commissure (AC). It lies anterior to the fornix, and mainly connects the two halves of the olfactory bulb and the temporal cortex. It is often used by callosally projecting neurons as an alternative route to reach the contralateral hemisphere in mutants where the corpus callosum fails to develop (Britanova et al., 2008; Chen et al., 2008; Livy et al., 1997). Several mouse mutants show abnormalities in formation of the AC, usually associated with a general failure of midline crossing (Armentano et al., 2006; Chen et al., 2007; Deuel et al., 2006). The Slit/Robo family of guidance cues and receptors seem to be essential in establishing a normal AC (Bagri et al., 2002; Kidd et al., 1998). 
Most commissural neurons originate from layer 2-4. The lack of anterior commissure in our mutants could be either due to a failure of midline crossing (which would also explain the formation of Probst bundles) or due to the failure of commissural neurons to extend axons towards the midline. The mechanism by which Sip1 might influence axonal crossing at the midline is yet to be determined.

\subsection{Sip1- mediated feedback signaling from postmitotic neurons to progenitor cells}

Our data on premature layer 2-5 production and precocious gliogenesis in a conditional mutant where Sip1 is specifically ablated only in the differentiating field of the cortex, and not the progenitors (Sip1fl/fl-Nex ${ }^{\mathrm{Cre}}$ ), suggest a non- cell autonomous role of Sip1 in regulating progenitor fate. Together with the fact that Sip1 is strongly expressed in the cortical plate, that absence of Sip1 leads to premature overexpression of secreted molecules NT3 and Fgf9 in the cortical plate at different timepoints in development, and that Fgf9 alone can induce gliogenic proliferation in the VZ in vitro, Sip1 certainly seems to induce a signal back to the cortical germinal zone to ensure sequential generation of cortical cell types (Fig21).

So far, not much evidence supporting neuron to progenitor feedback signaling as an extrinsic regulator of neuronal number/ progenitor fate in the cortex is available. Besides BDNF (discussed later), several experiments in the retina prove the existence of a celltype specific feedback signal that can prevent early progenitors from differentiating into early- born retinal cell types, and trigger a fate switch towards late- born cells. (reviewed in (Pearson and Doe, 2004). Interestingly, in ferret cortices, when layer 4 progenitors were specifically ablated at a certain timepoint of development by MAM treatment, no disruption in lamination was observed. Layer 4 neurons as well as subsequently born cell types seemed to be generated normally, implying that cortical progenitors can somehow sense the number of specific cell types (in this case, layer 4 cells) already generated in the cortex, and when inconsistencies are detected, they would extend the time of neurogenesis accordingly to make sure that eventually, a normal cortex is created. In this scenario, it is highly likely that this is mediated via feedback signaling from cortical neurons to precursors (Noctor et al., 1999). Sip1- mediated feedback signaling from postmitotic neurons to progenitor cells to regulate neuronal production is the first evidence supporting such a mechanism in the cortex. 
The ability of CP neurons to relay information on neuronal numbers to the germinal zone was first proposed more than a decade ago. The work involved a study of the production of corticospinal motor neurons (CSMNs) in reeler mice. These mutants are characterised by radial intermixing of cortical neurons. It was shown that in these mice, the rate of neuron production at early stages (when CSMN neurons are born) is lower than wildtype due to decreased mitotic exit. At the same time the probability that a cell born at this stage will become a CSMN is higher in reeler than in wildtype, so that eventually, there are significantly more CSMNs in the former. One possible explanation proposed by the authors suggested the presence of a negative feedback signal from the cortex to the VZ relayed via descending axons from cortical neurons. This signal would instruct the fate of cells during their final mitotis. In reeler, this signal is disrupted, possibly because of fewer cells present in the cortex. As a consequence, the inhibition is repressed and more CSMN neurons are formed within the same time window. In other words, the cortex senses the lack of CSMNs, signals back to progenitors so that the probability that a neuron born now will become a CSMN is higher, and in this way, makes up for the deficiency and eventually ends up with more corticospinal neurons than normal ((Polleux et al., 1998), reviewed in (Dehay and Kennedy, 2007)).

Some secreted cytokines/ chemokines expressed in the neocortex were also shown to be essential for cortical lamination. For instance, Stromal derived factor-1, which is normally expressed in the developing murine cortical plate, was shown to induce enhanced proliferation of late cortical progenitors and ectopic placement of neurons born thereafter (Liapi et al., 2008).

However, so far, only one instance has been reported where a signaling molecule secreted by postmitotic neurons was shown to influence the fate of cortical progenitors. Young neurons secrete the cytokine cardiotrophin-1 that can activate JAK-STAT signaling within early cortical precursors (that were shown to be responsive to certain gliogenic factors) and stimulate astrocytic differentiation. In vivo, CT-1 is expressed in the cortical plate during late corticogenesis, and mutation in CT-1 leads to decreased postnatal astrocytogenesis (Barnabe-Heider et al., 2005). Our studies demonstrate once again, the existence of a feedback signaling mechanism, mediated this time by transcription factor Sip1 and growth 
factor Fgf9, which not only regulates the level but also the onset of gliogenesis in the developing cortex.

\subsubsection{Cortical neurons secrete Fgf9 that signals back to the germinal zones to induce proliferation of astrocytic precursors and/or fate switch of neuronal progenitors towards a glial lineage}

The only member of the Fgf family of growth factors that has been repeatedly implicated in cortical astrocytogenesis, is Fgf2. It increases the competency of progenitors to respond to the astrocytic signal CNTF (Song and Ghosh, 2004). We have shown that Sip1 most likely exerts its gliogenic influence on cortical progenitor fate via FGF9 mediated signaling. Though Fgf9 was reported to be expressed in some CNS neurons including layer 3-5 neurons in the cortex, and glial cells of the spinal cord (Nakamura et al., 1999; Todo et al., 1998) no function of Fgf9 mediated signaling has so far been reported in cortical gliogenesis. However, in other systems such as the retina, Fgf9 is known to influence the lineage commitment of neural progenitors. It has been shown that when Fgf9 is ectopically expressed in early embryonic retinal pigment epithelium (RPE), it leads firstly, to an increased rate of proliferation in the RPE, and secondly, a cell fate switch whereby these cells lose their RPE character and become neural retina (Zhao et al., 2001). Also of interest is the observation that exogenous Fgf9 can induce proliferation of Müller glia in vitro, since the latter display a considerable level of expression of Fgfr2 and Fgfr3. In this case, Fgf9 is synthesised and secreted by retinal neurons that then activate the corresponding signaling pathway in retinal glial cells (Cinaroglu et al., 2005).

It is important to note here that although we find an increase in the expression of Fgfr3 in Sip1 mutants, and can therefore speculate that the precocious FGF9 signaling is mediated via its interaction with Fgfr3, in our in vitro experiments, we found exogenous FGF9 to induce a similar effect in wildtype cortices as well, at a stage when no endogenous Fgfr3 expression has been reported in the VZ (Bansal et al., 2003). Between E11.5 to E14.5, it is expressed in a high caudal to low rostral gradient (Fukuchi-Shimogori and Grove, 2003; Garel et al., 2003). At later stages (E16.5-E17.5) other Fgf receptors, Fgfr1 and Fgfr2, are also expressed in the cortex. While Fgfr1 was shown to be expressed in the VZ (Vaccarino et al., 1999), data from our collaborators indicates Fgfr2 expression in the VZ as well. Also, by SQ-PCR, we found the known isoform of Fgfr2 to be upregulated in cortical 
extracts at E16.5 (Fig13). Interestingly, in both Fgfr2-GFAP ${ }^{\text {Cre }}$ single conditional knockouts and Fgfr1/Fgfr2- GFAP ${ }^{\mathrm{Cre}}$ double mutants, there is a decrease in the density of astrocytes in the dorsal cortex (Smith et al., 2006). Moreover, at early stages, expression of activated Fgfr2 (expressed endogenously in radial glia) in the VZ causes cells of the ganglionic eminences (GE) to become radial glia and express RC2. It was also shown that activation of Notch signaling in GE cells expressing Fgfr2 endogenously, increases their responsiveness to Fgfs in culture. In other words, at early stages, in the GE, Notch activity improves Fgf responsiveness. Together with the fact that activated Notch itself induces radial glial identity and that these cells, at least in the cortex, become astrocytes postnatally, it is plausible that activation of Fgfr2 receptors by Fgf9 (both upregulated in Sip1 mutants) induces these cells to become astrocytic radial glia (Gaiano et al., 2000; Yoon et al., 2004). Therefore, Fgf9 might function via interaction with multiple receptors, not only Fgfr3 with which it supposedly forms the strongest interaction (Arnaud-Dabernat et al., 2007; Garofalo et al., 1999). Interestingly, Fgfr3 was shown to be expressed by astrocytes and their progenitors in the developing spinal cord, and contrary to our model, deletion of Fgfr3 led to an increase in astrocytogenesis in this system (Pringle et al., 2003). This report reflects the pleiotropic functions of Fgf signaling in the developing CNS. In cortical development, Fgfr3 has so far been implicated in controlling cortical size by regulating proliferation and apoptosis early in development (Inglis-Broadgate et al., 2005; Thomson et al., 2007). On the other hand, interfering with Fgfr1 signaling leads to decreased proliferation, abnormal radial glia, thinner cortex, and fewer layer 5/6 neurons (Ohkubo et al., 2004). GFAP-Cre mediated conditional knockout of Fgfr1 leads to lack of corpus callosum due to defective translocation of radial glia from cortical VZ to cortical midline. It has also been shown that exogenous Fgf8 can induce PCNA+Blbp+ radial glial cells to migrate into the cortical plate. It was also reported that the number of hippocampal radial glial progenitors is highly reduced in Fgfr1- GFAP ${ }^{\mathrm{Cre}}$ mice (Ohkubo et al., 2004). Our own preliminary studies with Sip1-Fgfr1 double conditional mutants revealed no genetic interaction between the two molecules, and no influence of Fgfr1 on early postnatal proliferation in the cortex (Fig14). This, in turn, could also be due to redundant functions of Fgfr1/2/3.

Our studies have also revealed the existence of an as yet unidentified splice variant of Fgfr2 in the neocortex. We have shown that it differs from known isoforms at least with regard to its 3' sequence, which seems to be transcribed from an unannotated exon within 
the 3' intronic sequence of Fgfr2 gene. Interestingly, while the known isoform of this receptor is strongly upregulated in Siplfl/fl-Emx ${ }^{\mathrm{Cre}}$, the new variant is starkly downregulated (Fig13). The significance of this observation with regard to the gliogenic phenotype in Sip1 mutants is yet to be determined.

\subsubsection{Premature expression of Neurotrophin-3 in Sip1- deficient cortical plate is not sufficient to induce progenitors to switch fate from generating deep layer neurons to upper layer neurons}

The neurotrophins BDNF and NT-3, alongwith their receptors TrkB and TrkC have been shown to be essential for survival and proliferation of cortical progenitors, as well as their differentiation into neurons. Inhibition of MEK-ERK mediated signaling downstream of Trk receptors led to inhibition of neurogenesis but had no effect on astrocytogenesis (Barnabe-Heider and Miller, 2003; Bartkowska et al., 2007). It has been shown that when overexpressed in cortical precursors, BDNF can promote premature neurogenesis in the VZ/SVZ, suggesting that once secreted, it can activate TrkB mediated signaling non- cell autonomously (Bartkowska et al., 2007). Since inhibition of Trk signaling can delay differentiation of precursors into neurons and hamper their migration to the appropriate location within the cortical plate (Bartkowska et al., 2007), as a corollary, we can expect increased Trk activation (due to upregulation of NT-3) in Sip1 mutants to cause the opposite effect. In other words, higher Trk signaling possibly induces premature neuronal differentiation of E12.5-E13.5 progenitors, which then manage to migrate to their appropriate location (layers 2-4) despite being born early. It was very intriguing to note that when VZ progenitors are exposed to BDNF applied to the lateral ventricle, cortical progenitors undergo a change in their lineage commitment, accelerate the completion of cell cycle, and differentiate into deep layer neurons rather than upper layer neurons. On the other hand, when endogenous BDNF is functionally blocked, upper layer neurons are born prematurely (Fukumitsu et al., 2006). We propose that NT-3, the other member of the same family of neurotrophins, does something similar, however, non- cell autonomously; in other words, when precociously secreted from cortical plate neurons, NT-3 influences the fate of $\mathrm{VZ}$ precursors towards an upper rather than deep layer neuronal type.

Much work has been done to explore the roles of NT3 in corticogenesis. It was shown that exogenous NT3, when added to E13.5-E15,5 cortical cells, can induce cell cycle exit, 
thereby decreasing proliferation and promoting differentiative cell division. It can also increase the duration of cell cycle (Lukaszewicz et al., 2002). Since the timing of mitotic exit determines the final cortical neuronal fate (Caviness et al., 1995; Polleux et al., 2001), any alterations in NT3 levels within the neocortex can be expected to influence fate determination of cortical progenitors. It is essential to keep in mind that NT3 is a secreted molecule and that $\mathrm{TrkC}$ receptors are distributed all over the cortex (data from collaborators Dr. Eve Seuntjens and Prof. Danny Huylebroeck, University of Leuven). However, we did not find any changes in cell cycle exit at E12.5-E13.5 in Sip1 mutants; the duration of the cell cycle seems to be unaltered as well (data from collaborators Dr. Eve Seuntjens and Prof. Danny Huylebroeck, University of Leuven). Also, in our in vitro experiments, neither high concentrations of NT3 in the medium nor localised application through Affigel beads could induce any notable shift in the ratio of upper to deep layer neurons within the early cortex (Fig16). Therefore, it seems unlikely that NT3 alone acts directly on cortical progenitors to influence cell cycle kinetics and hence cell fate. It is more probable that the function of NT3 is mediated via or in combination with other signaling molecules/pathways that eventually trigger specific cascades within cells of the $\mathrm{VZ} / \mathrm{SVZ}$ and induce them to switch fates precociously.

\subsubsection{Differentiation of early cortical progenitors on older wildtype and mutant cortices}

We have shown that in our slice overlay assays E12.5 cortical cells have atleast twice less tendency to differentiate into Satb2+ cells on an E15.5 cortical slice than on an E13.5 cortex. To avoid tracing the fate of already committed precursors, we focused specifically on cells that were in the cell cycle at the time of plating. One likely explanation could be that some of the plated cells, although committed towards an upper layer fate, still need an early stage cortical plate to complete their differentiation. On an E15.5 microenvironment, although committed, they cannot differentiate into Satb2+ cells, probably because the cues needed for E15.5 precursors to become upper layer neurons are different from the cues needed for E13.5 precursors to do the same (Fig18). Furthermore, we also show that E13.5 cortical cells (a stage too early for astrocytic commitment in vivo) differentiate into astrocytes less frequently when plated on E17.5 mutant slices than on wildtype slices of the same age (Fig19). This observation, at first glance, seems contradictory to our hypothesis that Sip1 deficient cortical plate is more conducive to astrocytogenesis. However, in our 
conditional mutants, astrocytic precursors are specified prematurely, which would suggest that the gliogenic signal in Sip1 mutants reaches peak levels earlier than in wildtype (E17.5). The increased proliferation seen at this stage is probably a consequence of the enhanced signaling that occurs a day earlier. Once triggered to proliferate, a certain fraction of astrocytic precursors continue dividing until early postnatal stages. Though not confirmed, it is possible that the amount of gliogenic signal in E17.5 mutant cortices is lesser than in wildtype cortices. Hence, we detect decreased tendency of E13.5 cells to differentiate into astrocytes on mutant slices. Culturing these cells on E16.5 wildtype and mutant slices is perhaps the best alternative.

\subsection{Regulatory signaling pathways influenced by Sip1}

Several signaling pathways have been implicated in cortical astrogliogenesis. These include members of the IL-6 family of cytokines, LIF and CNTF, that exert their effects via JAK/STAT signaling; Notch receptor- Delta ligand; BMPs, especially BMP2/4/7, that act via phosphorylation of Smad1/5/8 and possibly crosstalk with Notch signaling; Fgf2, and EGF among others. According to our data, Sip1 clearly has no detectable influence on the level of Notch signaling either at early or late stages of corticogenesis, though it seems to regulate levels of Fgf- and BMP- mediated signaling.

\subsubsection{Gliogenic feedback signaling in the absence of Sip1 induces BMP activity in abventricular astrocytic precursors at E17.5}

In Sip1fl/fl-Emx ${ }^{\text {Cre }}$, we have shown ectopic BMP activity in cells dispersed within the cortical germinal zone but away from the VZ, at E17.5 (Fig17). It is important at this point to reiterate the fact that in Sip1 mutants, at precisely the same developmental stage, we could detect increased number of dispersed astrocytic precursors. Although, due to technical difficulties, we could not colocalise p-Smad1/5/8 immunostaining with proliferation markers, it is highly likely that they indeed are coexpressed. This leads to the following possibility- absence of Sip1 in the cortical plate induces Fgf9 signaling, which in turn activates BMP activity in the VZ/SVZ, that expectedly, switches progenitor fate towards an astrocytic lineage. If this indeed is true, then it would be the first time that a crosstalk between BMP and FGF signaling is being reported in the cortex. Whether the interaction between the two pathways is direct or indirect, is purely upto speculation. 
Although Sip1 protein could never be detected in the germinal zones, in situ hybridization based detection of Sip1 transcripts has suggested weak expression in the VZ/SVZ. This in turn suggests that in Sip1fl/fl-Emx ${ }^{\text {Cre }}$, lack of this low Sip1 level might directly activate BMP signaling in some cells of the VZ. We proposed earlier that in the presumptive hippocampus, Sip1 forms a transcriptional repressor complex alongwith BMP-Smads (Miquelajauregui et al., 2007), which would suggest that in the absence of Sip1, the transcriptional activation function of BMP Smads might actually increase, which could very well be the case in the neocortex as well.

Interestingly, it was shown that when sympathetic superior cervical ganglia were treated with BMP2, not only did it increase the levels of TrkC mRNA, but it also increased responsiveness to neurotrophin-3. Although at early stages, we did not detect any substantial changes in p-Smad1/5/8 levels based on immunostainings, we cannot preclude BMP-NT3 interactions as a cause of defective lamination in the conditional knockouts. In other words, the DL to UL cell fate switch during early corticogenesis might be mediated by increased NT3 levels alongwith increased BMP activity, which might together enhance the responsiveness of endogenous TrkC receptors to NT3. This would also explain why in our various in vitro experiments, NT3 alone could never induce increased production of upper layer neurons.

\subsection{Molecular targets of Sip1 in the neocortex- transcription factors, growth factors/cytokines and cell adhesion molecules among others}

In order to explore the molecular pathways activated/ repressed by the presence/absence of Sip1, we compared gene expression between wildtype and Sip1fl/fl-Emx ${ }^{\mathrm{Cre}}$ cortices using microarray and SQ-PCR (Fig13). We selected the following putative Sip1 targets for further analysis-

1. Foxo1, a forkhead domain containing transcription factor, upregulated in Sip1 mutants at E14.5 and E16.5. It is involved in controlling cell cycle progression (Kops et al., 2002; Schmidt et al., 2002), and cell death (Barthelemy et al., 2004), among other functions. Interestingly, Foxo proteins have been shown to form transcriptional activator complexes (to activate expression of $\mathrm{Cdk}$ inhibitors) with $\mathrm{Smad} 2$ and Smad4 upon stimulation by 
TGFß (Seoane et al., 2004). Expression of Foxo1 in the developing mouse neocortex has so far not been reported. (Hoekman et al., 2006).

2. Cbln4 (Cerebellin 4 precursor), a cytokine upregulated in Sip1 mutants at E14.5. It is reportedly expressed in the entorrhinal cortex and in cortical layer 4 in adult mice. Expression of other members of this family, Cbln 1 and $\mathrm{Cb} \ln 2$, is more extensive in the cortex during embryonic development and early postnatal stages (Miura et al., 2006). Interestingly, we found an upregulation of STAT4 as well at this stage. STAT4 is a downstream effector of cytokine- mediated signaling cascades (IFN $/ /$, IL-12,23) (Kaplan, 2005). Although no functions of STAT4 have been reported till date in cortical development, it is quite possible that it acts downstream of Cbln4 and that together, they influence gene expression in cortical progenitors. Since by E14.5, the production of upper layer neurons is almost complete in Sip1 mutants, the enhanced Cbln4- STAT4 mediated signaling might also be involved in enabling germinal zone precursors to sense the relative numbers of upper and deep layer neurons already produced in the cortex and thereby instruct them to switch fates to the next cell type that has to be generated.

3. Itga6 (Integrin- $\alpha 6$ ), downregulated at E13.5 and E14.5 but upregulated at E16.5. Possible roles of Itga6 in cortical development have been discussed before.

4. Ebf1 (early B-cell factor1), a transcription factor upregulated in Sip1 mutants at E14.5. In the neocortex, so far, it has been reported to be expressed only in the marginal zone; however, in other regions of the brain it might play a role in neuronal differentiation (Garel et al., 1999). Studies in the chick embryo have further corroborated its essential role in neuronal subtype specification and stabilisation of the committed state of progenitors, possibly downstream to proneural genes. It also seems to be essential for initiating migration of neuroepithelial cells in chick (Garcia-Dominguez et al., 2003). Hence, the increased expression of Ebf1 in Sip1 mutants may strengthen upper layer commitment of cells born before E15.5, even if they were meant to be deep layer neurons. Phenotypic analysis of Sip1 mutants revealed that Satb2+ and Brn2+ neurons born prematurely manage to migrate correctly to the upper layers of the cortical plate already by E15.5; this implies that while in wildtype cortices, early born cells migrate to only the deep layers by this time, in mutants, they migrate faster. The early upregulation of factors like Ebf1 might facilitate this process in Sip1 mutants. 
5. Elovl7 (elongation of long chain fatty acids), upregulated at E14.5, codes for an elongase essential for lipid metabolism (Jakobsson et al., 2006). It is interesting to note that Elovl7 expression is almost undetectable in the wildtype at E14.5, while in the mutant, it seems to be strongly upregulated. Whether this is a cause or a consequence of altered cortical lamination, is currently upto speculation. It is also possible that Elovl7 is expressed specifically in a subpopulation of upper layer neurons, which are expanded in Sip1 mutants at prenatal stages. Elovl7 could thus be a new, as yet uncharacterised layer- specific marker.

6. Ldb3 (LIM- domain binding protein 3), upregulated at E14.5. It is known to be important for integrin function and for assembly of integrin adhesion sites in Drosophila (Jani and Schock, 2007). The related family member, Ldb1, is essential for anteroposterior axial patterning in post- gastrulation mouse embryos (Mukhopadhyay et al., 2003). It has also been suggested that there could be a certain level of redundancy between the different members of this family (Matthews and Visvader, 2003). Interestingly, in Ldb1 mutants, there is a reduction in the expression of Wnt inhibitor Sfrp1 as early as E7.5, while in Sip1 mutants, where Ldb3 is strongly upregulated, Sfrp1 expression is upregulated as well.

7. Ripk4, a serine/threonine kinase, upregulated at E14.5. Ripk4 overexpression was shown to activate JNK signaling in HeLa cells (Meylan et al., 2002). Since JNK activity has the potential to hamper migration of E16.5 neuronal precursors (Mizuno et al., 2005), it is possible that increased Ripk4 activity in Sip1 mutants might, through activation of JNK, inhibit the migration of late born neurons.

8. Wnt5a, upregulated at E14.5. Although Wnt5a is expressed in the cortical hem (Grove et al., 1998), its function in corticogenesis has not yet been reported. Interestingly, the hem is not affected in either Sip1 conditional mutants. First of all, neither Emx1 nor Nex promoter is active in this region (Goebbels et al., 2006; Yoshida et al., 1997). Secondly, expression of Wnt3a and Wnt5a in the hem is reportedly unchanged in Sip1fl/fl-Emx ${ }^{\mathrm{Cre}}$ (Doctoral Thesis, Miquelajauregui A, Mar 2006, University of Goettingen, unpublished data).

9. Robo1, downregulated at E13.5. Robo1 is an axon guidance receptor that, in combination with secreted Slit ligands, prevents axons from inappropriately crossing the 
CNS midline. In the cortex, Robol was found to be essential in forming axonal tracts mostly during late corticogenesis. It is expressed in callosal axons and the hippocampal commissure at E17.5. At early stages, while some reports show expression of Robo1 protein in the MZ and the lower IZ at E15.5 (Andrews et al., 2006), others showed the corresponding transcripts at E13.5 in the cortical plate (Lopez-Bendito et al., 2007). Moreover, there are contrasting reports on the function of Robol in corticogenesis. In one report, callosal and hippocampal axons in Robol knockout mice form tight fascicles and fail to cross the midline. When analysed at E14.5, both thalamocortical as well as corticofugal axons were found to advance faster towards their target in comparison to the wildtype. Also, in the absence of Robo1, interneurons migrate faster into the cortex as early as E12.5 (Andrews et al., 2006). In the second report, only very minor defects in callosal connections were found in Robo1 mutants. Only Robo1/2 double knockouts displayed severe axon guidance defects(Lopez-Bendito et al., 2007). However, so far, no role of Robol has been reported in cortical lamination.

10. Gmfß, upregulated at E16.5. Gmfß, when overexpressed in astrocytes, promotes proliferation and morphological differentiation (Lim et al., 1989). It is expressed on the surface of astrocytes, and also stored inside, though not secreted. It functions intracellularly as a modulator of MAP kinase signaling (Lim et al., 1990; Zaheer et al., 2001). Not much is known about the role of GMFß in cortical development. Since we have shown increased numbers of astrocytic precursors in Sip1 mutants at E17.5, and upregulation in GMFß levels in the cortex one day earlier, it is likely that GMFß expression increases within cells that switch fate, and that will proliferate further and differentiate into astrocytes.

11. PCdh9 (protocadherin 9), upregulated at E16.5. PCdh9 is a cell- cell adhesion molecule shown to be expressed in the $\mathrm{CP}$ and IZ at E18.5 in rats and almost all over the cortex at P3 in the region of parietal and occipital cortex (Kim et al., 2007; Vanhalst et al., 2005). Though no function of PCdh9 has been reported yet in cortical development, it may contribute to the hampered migration of late born neurons in Sip1 mutants. 
4.7 Mixed identity and/or delayed maturation of some layer 5/6 cortical neurons in Sip1 conditional mutants

Our birthdating experiments indicate that the dynamics of production of layer 6 neurons (Tbr1+) is not affected in Sip1 mutants, though that of subsequently born cell types is. It has been shown that Tbr1+ cells are amongst the earliest to be born from cortical progenitors. Expression of Tbr1 protein in the preplate can be detected as early as E12.5 while its transcripts are already seen at E10.5 as soon as the first cortical cells begin to differentiate. Subsequently, cells born at E11.5 show a high level of Tbr1 expression (Hevner et al., 2001). Similarly, Sox5+ cells are also most likely to be amongst the earliest born cell types of the cortex since in their absence, even the subplate neurons born at E11.5 migrate abnormally to superficial layers and express Ctip2 which is normally repressed in this population by Sox 5 (Lai et al., 2008). Also, activity of Sox 5 is upstream to Fezl, the master gene required for the generation of subcerebral projection neurons (Kwan et al., 2008; Molyneaux et al., 2005). The transcription factor Foxp1 is mostly expressed in layers 3-5 with weak expression in some cells of layer 6. Based on the dynamics of expression of Sox 5 and Foxp1, the former predominantly in layer 6 but also in layer 5 and the latter mostly in layer 5, we can say that Sox 5+ neurons are generated prior to Foxp1+ neurons. Although, due to technical difficulties, we could not perform BrdU pulse- chase experiments to check the temporal sequence of production of Sox5+ cells in Sip1 mutants, we did find Foxp1+ cells to be produced precociously, similar to the Ctip2+ population. Interestingly, we found more cells expressing both Sox 5 and Foxp1 in layers 5/6 in Sip1f1/fl-Nex ${ }^{\mathrm{Cre}}$ at E17.5, cells expressing both Tbr1 and Ctip2 in Sip1fl/fl-Emx ${ }^{\mathrm{Cre}}$ at E16.5 and cells expressing both Satb2 and Ctip2 in Sip1fl/fl-Nex ${ }^{\mathrm{Cre}}$ at E15.5 (Fig5). The maturation of Ctip2+Tbr1+ cells into Ctip2+Tbr1- cells, and that of Foxp2+Sox5+ cells into Foxp2+Sox5- cells seems to be hampered. We believe that probably as a consequence of the fate switch at E12.5, a cohort of early born deep layer neurons possess a confused/mixed identity, at least with regard to the expression of certain layer- specific molecular markers. It has been reported previously that cells expressing both Satb2 and Ctip2 tend to lose Ctip2 expression due to Satb2- mediated repression and become upper layer neurons (Britanova et al., 2008). Our data indicate that although UL neurons are specified earlier in Sip1 mutants, the maturation of some of these cells (Satb2 expression) seems to be delayed in comparison to the wildtype. This in turn could be due to the 'confusion' created in the mutant cortical plate due to the lack of neurons with DL 
characteristics (Ctip2, Tbr1, Foxp1, Sox5 expression) and the precocious appearance of neurons with UL features (Brn2 expression).

\subsection{Upregulation of Wnt antagonist Sfrp1 is not responsible for enhanced gliogenesis in $\operatorname{Sip} 1$ conditional mutants}

It was reported previously that at E17.5, Sfrp1 is strongly upregulated in the mutant cortical plate, whereas in the wildtype, its expression is restricted to the VZ/SVZ (Doctoral Thesis, Miquelajauregui A, Mar 2006, University of Goettingen, unpublished data). Also, Sip1 can directly repress its expression in the cortex (Miquelajauregui et al., 2007). This secreted Wnt antagonist was therefore, a promising candidate downstream of Sip1, to mediate the latter's gliogenic effects. However we have shown that deletion of Sfrp1 in Sip1 conditional mutants neither rescues the enhanced gliogenesis and postnatal proliferation nor the reduction in deep layers seen in these mutants (Fig20). As a corollary we also explored the effects of overexpressing Sfrp1 in the embryonic cortical VZ, and found no alterations in GFAP expression at birth (Fig20). Altogether, our data suggest that Sfrp1 alone is probably not responsible for inducing cell fate switch in the cortical progenitors, either at early or late corticogenesis. There is most likely an interplay of several molecules/signaling pathways expressed/activated as a consequence of Sip1 ablation, which in turn leads to the diverse array of phenotypic effects seen in the conditional mutants. 


\section{CONCLUSION}

Several independent reports in the past have shown that a single multipotent cortical progenitor cell can first give rise to several types of layer- specific neurons, followed by astrocytes in a temporally specified sequence of events. This sequence is not only regulated by intrinsic determinants of neuronal versus glial fate, but also by epigenetic and extrinsic cues. Our work with conditional ablation of transcriptional repressor Sip1 exclusively in the postmitotic neurons of the cortex has shown that, during embryonic development of the neocortex, young neurons signal back to uncommitted progenitors to regulate the generation time and the numbers of different cortical cell types. In the absence of the highly expressed Sip1, this feedback mechanism is mediated via Fgf9 to control the neuron versus glia fate of precursors during late corticogenesis, and possibly via NT3 in combination with other as yet unconfirmed factor(s) to control deep versus upper layer neuron fate of progenitors during early corticogenesis. 


\section{REFERENCES}

Alcamo, E.A., Chirivella, L., Dautzenberg, M., Dobreva, G., Farinas, I., Grosschedl, R., and McConnell, S.K. (2008). Satb2 regulates callosal projection neuron identity in the developing cerebral cortex. Neuron 57, 364-377.

Andrews, W., Liapi, A., Plachez, C., Camurri, L., Zhang, J., Mori, S., Murakami, F., Parnavelas, J.G., Sundaresan, V., and Richards, L.J. (2006). Robo1 regulates the development of major axon tracts and interneuron migration in the forebrain. Development 133, 2243-2252.

Anton, E.S., Kreidberg, J.A., and Rakic, P. (1999). Distinct functions of alpha3 and alpha(v) integrin receptors in neuronal migration and laminar organization of the cerebral cortex. Neuron 22, 277-289.

Arlotta, P., Molyneaux, B.J., Chen, J., Inoue, J., Kominami, R., and Macklis, J.D. (2005). Neuronal subtype-specific genes that control corticospinal motor neuron development in vivo. Neuron 45, 207-221.

Armentano, M., Filosa, A., Andolfi, G., and Studer, M. (2006). COUP-TFI is required for the formation of commissural projections in the forebrain by regulating axonal growth. Development $133,4151-4162$.

Arnaud-Dabernat, S., Kritzik, M., Kayali, A.G., Zhang, Y.Q., Liu, G., Ungles, C., and Sarvetnick, N. (2007). FGFR3 is a negative regulator of the expansion of pancreatic epithelial cells. Diabetes 56, 96-106.

Arnold, S.J., Huang, G.J., Cheung, A.F., Era, T., Nishikawa, S., Bikoff, E.K., Molnar, Z., Robertson, E.J., and Groszer, M. (2008). The T-box transcription factor Eomes/Tbr2 regulates neurogenesis in the cortical subventricular zone. Genes Dev 22, 2479-2484.

Bagri, A., Marin, O., Plump, A.S., Mak, J., Pleasure, S.J., Rubenstein, J.L., and Tessier-Lavigne, M. (2002). Slit proteins prevent midline crossing and determine the dorsoventral position of major axonal pathways in the mammalian forebrain. Neuron 33, 233-248.

Bai, J., Ramos, R.L., Ackman, J.B., Thomas, A.M., Lee, R.V., and LoTurco, J.J. (2003). RNAi reveals doublecortin is required for radial migration in rat neocortex. Nat Neurosci 6, 1277-1283.

Bansal, R., Lakhina, V., Remedios, R., and Tole, S. (2003). Expression of FGF receptors 1, 2, 3 in the embryonic and postnatal mouse brain compared with Pdgfralpha, Olig2 and Plp/dm20: implications for oligodendrocyte development. Dev Neurosci 25, 83-95.

Barnabe-Heider, F., and Miller, F.D. (2003). Endogenously produced neurotrophins regulate survival and differentiation of cortical progenitors via distinct signaling pathways. J Neurosci 23, 5149-5160. 
Barnabe-Heider, F., Wasylnka, J.A., Fernandes, K.J., Porsche, C., Sendtner, M., Kaplan, D.R., and Miller, F.D. (2005). Evidence that embryonic neurons regulate the onset of cortical gliogenesis via cardiotrophin-1. Neuron 48, 253-265.

Barthelemy, C., Henderson, C.E., and Pettmann, B. (2004). Foxo3a induces motoneuron death through the Fas pathway in cooperation with JNK. BMC Neurosci 5, 48.

Bartkowska, K., Paquin, A., Gauthier, A.S., Kaplan, D.R., and Miller, F.D. (2007). Trk signaling regulates neural precursor cell proliferation and differentiation during cortical development. Development 134, 4369-4380.

Bayer, S.A., Altman, J. (1991). Neocortical Development (Raven Press).

Bielle, F., Griveau, A., Narboux-Neme, N., Vigneau, S., Sigrist, M., Arber, S., Wassef, M., and Pierani, A. (2005). Multiple origins of Cajal-Retzius cells at the borders of the developing pallium. Nat Neurosci 8, 1002-1012.

Bishop, K.M., Goudreau, G., and O'Leary, D.D. (2000). Regulation of area identity in the mammalian neocortex by Emx2 and Pax6. Science 288, 344-349.

Bodine, P.V., Zhao, W., Kharode, Y.P., Bex, F.J., Lambert, A.J., Goad, M.B., Gaur, T., Stein, G.S., Lian, J.B., and Komm, B.S. (2004). The Wnt antagonist secreted frizzled-related protein-1 is a negative regulator of trabecular bone formation in adult mice. Mol Endocrinol 18, 1222-1237.

Bonaguidi, M.A., McGuire, T., Hu, M., Kan, L., Samanta, J., and Kessler, J.A. (2005). LIF and BMP signaling generate separate and discrete types of GFAP-expressing cells. Development 132, 5503-5514.

Bonni, A., Sun, Y., Nadal-Vicens, M., Bhatt, A., Frank, D.A., Rozovsky, I., Stahl, N., Yancopoulos, G.D., and Greenberg, M.E. (1997). Regulation of gliogenesis in the central nervous system by the JAK-STAT signaling pathway. Science 278, 477-483.

Britanova, O., Akopov, S., Lukyanov, S., Gruss, P., and Tarabykin, V. (2005). Novel transcription factor Satb2 interacts with matrix attachment region DNA elements in a tissue-specific manner and demonstrates cell-type-dependent expression in the developing mouse CNS. Eur J Neurosci 21, 658-668.

Britanova, O., de Juan Romero, C., Cheung, A., Kwan, K.Y., Schwark, M., Gyorgy, A., Vogel, T., Akopov, S., Mitkovski, M., Agoston, D., et al. (2008). Satb2 is a postmitotic determinant for upper-layer neuron specification in the neocortex. Neuron 57, 378-392.

Bulchand, S., Subramanian, L., and Tole, S. (2003). Dynamic spatiotemporal expression of LIM genes and cofactors in the embryonic and postnatal cerebral cortex. Dev Dyn 226, 460-469.

Bulfone, A., Smiga, S.M., Shimamura, K., Peterson, A., Puelles, L., and Rubenstein, J.L. (1995). T-brain-1: a homolog of Brachyury whose expression defines molecularly distinct domains within the cerebral cortex. Neuron 15, 63-78. 
Burrows, R.C., Wancio, D., Levitt, P., and Lillien, L. (1997). Response diversity and the timing of progenitor cell maturation are regulated by developmental changes in EGFR expression in the cortex. Neuron 19, 251-267.

Cai, J., Chen, Y., Cai, W.H., Hurlock, E.C., Wu, H., Kernie, S.G., Parada, L.F., and Lu, Q.R. (2007). A crucial role for Olig2 in white matter astrocyte development. Development 134, 18871899.

Cai, L., Hayes, N.L., Takahashi, T., Caviness, V.S., Jr., and Nowakowski, R.S. (2002). Size distribution of retrovirally marked lineages matches prediction from population measurements of cell cycle behavior. J Neurosci Res 69, 731-744.

Calegari, F., Haubensak, W., Haffner, C., and Huttner, W.B. (2005). Selective lengthening of the cell cycle in the neurogenic subpopulation of neural progenitor cells during mouse brain development. J Neurosci 25, 6533-6538.

Calegari, F., and Huttner, W.B. (2003). An inhibition of cyclin-dependent kinases that lengthens, but does not arrest, neuroepithelial cell cycle induces premature neurogenesis. J Cell Sci 116, 49474955.

Caviness, V.S., Jr., Takahashi, T., and Nowakowski, R.S. (1995). Numbers, time and neocortical neuronogenesis: a general developmental and evolutionary model. Trends Neurosci 18, 379-383.

Chambers, C.B., Peng, Y., Nguyen, H., Gaiano, N., Fishell, G., and Nye, J.S. (2001). Spatiotemporal selectivity of response to Notch1 signals in mammalian forebrain precursors. Development 128, 689-702.

Chen, B., Wang, S.S., Hattox, A.M., Rayburn, H., Nelson, S.B., and McConnell, S.K. (2008). The Fezf2-Ctip2 genetic pathway regulates the fate choice of subcortical projection neurons in the developing cerebral cortex. Proc Natl Acad Sci U S A 105, 11382-11387.

Chen, L., Liao, G., Waclaw, R.R., Burns, K.A., Linquist, D., Campbell, K., Zheng, Y., and Kuan, C.Y. (2007). Rac1 controls the formation of midline commissures and the competency of tangential migration in ventral telencephalic neurons. J Neurosci 27, 3884-3893.

Chenn, A., and Walsh, C.A. (2002). Regulation of cerebral cortical size by control of cell cycle exit in neural precursors. Science 297, 365-369.

Cinaroglu, A., Ozmen, Y., Ozdemir, A., Ozcan, F., Ergorul, C., Cayirlioglu, P., Hicks, D., and Bugra, K. (2005). Expression and possible function of fibroblast growth factor 9 (FGF9) and its cognate receptors FGFR2 and FGFR3 in postnatal and adult retina. J Neurosci Res 79, 329-339.

Comijn, J., Berx, G., Vermassen, P., Verschueren, K., van Grunsven, L., Bruyneel, E., Mareel, M., Huylebroeck, D., and van Roy, F. (2001). The two-handed E box binding zinc finger protein SIP1 downregulates E-cadherin and induces invasion. Mol Cell 7, 1267-1278.

Dahl, D., and Bignami, A. (1973). Immunochemical and immunofluorescence studies of the glial fibrillary acidic protein in vertebrates. Brain Res 61, 279-293. 
Dehay, C., and Kennedy, H. (2007). Cell-cycle control and cortical development. Nat Rev Neurosci $8,438-450$.

Delalande, J.M., Guyote, M.E., Smith, C.M., and Shepherd, I.T. (2008). Zebrafish sip1a and sip1b are essential for normal axial and neural patterning. Dev Dyn 237, 1060-1069.

Desai, A.R., and McConnell, S.K. (2000). Progressive restriction in fate potential by neural progenitors during cerebral cortical development. Development 127, 2863-2872.

Deuel, T.A., Liu, J.S., Corbo, J.C., Yoo, S.Y., Rorke-Adams, L.B., and Walsh, C.A. (2006). Genetic interactions between doublecortin and doublecortin-like kinase in neuronal migration and axon outgrowth. Neuron 49, 41-53.

Doetsch, F., Caille, I., Lim, D.A., Garcia-Verdugo, J.M., and Alvarez-Buylla, A. (1999). Subventricular zone astrocytes are neural stem cells in the adult mammalian brain. Cell 97, 703716.

Eisaki, A., Kuroda, H., Fukui, A., and Asashima, M. (2000). XSIP1, a member of two-handed zinc finger proteins, induced anterior neural markers in Xenopus laevis animal cap. Biochem Biophys Res Commun 271, 151-157.

Englund, C., Fink, A., Lau, C., Pham, D., Daza, R.A., Bulfone, A., Kowalczyk, T., and Hevner, R.F. (2005). Pax6, Tbr2, and Tbr1 are expressed sequentially by radial glia, intermediate progenitor cells, and postmitotic neurons in developing neocortex. J Neurosci 25, 247-251.

Feng, L., Hatten, M.E., and Heintz, N. (1994). Brain lipid-binding protein (BLBP): a novel signaling system in the developing mammalian CNS. Neuron 12, 895-908.

Fishell, G., and Hanashima, C. (2008). Pyramidal neurons grow up and change their mind. Neuron $57,333-338$

Fishell, G., Mason, C.A., and Hatten, M.E. (1993). Dispersion of neural progenitors within the germinal zones of the forebrain. Nature 362, 636-638.

Fukuchi-Shimogori, T., and Grove, E.A. (2003). Emx2 patterns the neocortex by regulating FGF positional signaling. Nat Neurosci 6, 825-831.

Fukumitsu, H., Ohtsuka, M., Murai, R., Nakamura, H., Itoh, K., and Furukawa, S. (2006). Brainderived neurotrophic factor participates in determination of neuronal laminar fate in the developing mouse cerebral cortex. J Neurosci 26, 13218-13230.

Gaiano, N., Nye, J.S., and Fishell, G. (2000). Radial glial identity is promoted by Notch1 signaling in the murine forebrain. Neuron 26, 395-404.

Garcia-Dominguez, M., Poquet, C., Garel, S., and Charnay, P. (2003). Ebf gene function is required for coupling neuronal differentiation and cell cycle exit. Development 130, 6013-6025.

Garel, S., Huffman, K.J., and Rubenstein, J.L. (2003). Molecular regionalization of the neocortex is disrupted in Fgf8 hypomorphic mutants. Development 130, 1903-1914.

Garel, S., Marin, F., Grosschedl, R., and Charnay, P. (1999). Ebf1 controls early cell differentiation in the embryonic striatum. Development 126, 5285-5294. 
Garofalo, S., Kliger-Spatz, M., Cooke, J.L., Wolstin, O., Lunstrum, G.P., Moshkovitz, S.M., Horton, W.A., and Yayon, A. (1999). Skeletal dysplasia and defective chondrocyte differentiation by targeted overexpression of fibroblast growth factor 9 in transgenic mice. J Bone Miner Res 14, 1909-1915.

Ge, W., Martinowich, K., Wu, X., He, F., Miyamoto, A., Fan, G., Weinmaster, G., and Sun, Y.E. (2002). Notch signaling promotes astrogliogenesis via direct CSL-mediated glial gene activation. $\mathrm{J}$ Neurosci Res 69, 848-860.

Georges-Labouesse, E., Mark, M., Messaddeq, N., and Gansmuller, A. (1998). Essential role of alpha 6 integrins in cortical and retinal lamination. Curr Biol 8, 983-986.

Gerdes, J., Schwab, U., Lemke, H., and Stein, H. (1983). Production of a mouse monoclonal antibody reactive with a human nuclear antigen associated with cell proliferation. Int $\mathrm{J}$ Cancer 31 , $13-20$

Gilmore, E.C., Ohshima, T., Goffinet, A.M., Kulkarni, A.B., and Herrup, K. (1998). Cyclindependent kinase 5-deficient mice demonstrate novel developmental arrest in cerebral cortex. $\mathrm{J}$ Neurosci 18, 6370-6377.

Goebbels, S., Bormuth, I., Bode, U., Hermanson, O., Schwab, M.H., and Nave, K.A. (2006). Genetic targeting of principal neurons in neocortex and hippocampus of NEX-Cre mice. Genesis $44,611-621$.

Gorski, J.A., Talley, T., Qiu, M., Puelles, L., Rubenstein, J.L., and Jones, K.R. (2002). Cortical excitatory neurons and glia, but not GABAergic neurons, are produced in the Emx1-expressing lineage. J Neurosci 22, 6309-6314.

Gotz, M., and Huttner, W.B. (2005). The cell biology of neurogenesis. Nat Rev Mol Cell Biol 6, 777-788.

Gotz, M., Stoykova, A., and Gruss, P. (1998). Pax6 controls radial glia differentiation in the cerebral cortex. Neuron 21, 1031-1044.

Grandbarbe, L., Bouissac, J., Rand, M., Hrabe de Angelis, M., Artavanis-Tsakonas, S., and Mohier, E. (2003). Delta-Notch signaling controls the generation of neurons/glia from neural stem cells in a stepwise process. Development 130, 1391-1402.

Gross, R.E., Mehler, M.F., Mabie, P.C., Zang, Z., Santschi, L., and Kessler, J.A. (1996). Bone morphogenetic proteins promote astroglial lineage commitment by mammalian subventricular zone progenitor cells. Neuron 17, 595-606.

Grove, E.A., Tole, S., Limon, J., Yip, L., and Ragsdale, C.W. (1998). The hem of the embryonic cerebral cortex is defined by the expression of multiple Wnt genes and is compromised in Gli3deficient mice. Development 125, 2315-2325.

Guillemot, F., Molnar, Z., Tarabykin, V., and Stoykova, A. (2006). Molecular mechanisms of cortical differentiation. Eur J Neurosci 23, 857-868. 
Hardonk, M.J., and Harms, G. (1990). The use of 5'-bromodeoxyuridine in the study of cell proliferation. Acta Histochem Suppl 39, 99-108.

Hartfuss, E., Galli, R., Heins, N., and Gotz, M. (2001). Characterization of CNS precursor subtypes and radial glia. Dev Biol 229, 15-30.

Haubensak, W., Attardo, A., Denk, W., and Huttner, W.B. (2004). Neurons arise in the basal neuroepithelium of the early mammalian telencephalon: a major site of neurogenesis. Proc Natl Acad Sci U S A 101, 3196-3201.

He, F., Ge, W., Martinowich, K., Becker-Catania, S., Coskun, V., Zhu, W., Wu, H., Castro, D., Guillemot, F., Fan, G., et al. (2005). A positive autoregulatory loop of Jak-STAT signaling controls the onset of astrogliogenesis. Nat Neurosci 8, 616-625.

Hendzel, M.J., Wei, Y., Mancini, M.A., Van Hooser, A., Ranalli, T., Brinkley, B.R., Bazett-Jones, D.P., and Allis, C.D. (1997). Mitosis-specific phosphorylation of histone H3 initiates primarily within pericentromeric heterochromatin during G2 and spreads in an ordered fashion coincident with mitotic chromosome condensation. Chromosoma 106, 348-360.

Hermanson, O., Jepsen, K., and Rosenfeld, M.G. (2002). N-CoR controls differentiation of neural stem cells into astrocytes. Nature 419, 934-939.

Hevner, R.F., Miyashita-Lin, E., and Rubenstein, J.L. (2002). Cortical and thalamic axon pathfinding defects in Tbr1, Gbx2, and Pax6 mutant mice: evidence that cortical and thalamic axons interact and guide each other. J Comp Neurol 447, 8-17.

Hevner, R.F., Shi, L., Justice, N., Hsueh, Y., Sheng, M., Smiga, S., Bulfone, A., Goffinet, A.M., Campagnoni, A.T., and Rubenstein, J.L. (2001). Tbr1 regulates differentiation of the preplate and layer 6. Neuron 29, 353-366.

Higashi, Y., Maruhashi, M., Nelles, L., Van de Putte, T., Verschueren, K., Miyoshi, T., Yoshimoto, A., Kondoh, H., and Huylebroeck, D. (2002). Generation of the floxed allele of the SIP1 (Smadinteracting protein 1) gene for Cre-mediated conditional knockout in the mouse. Genesis 32, 82-84. Hockfield, S., and McKay, R.D. (1985). Identification of major cell classes in the developing mammalian nervous system. J Neurosci 5, 3310-3328.

Hoekman, M.F., Jacobs, F.M., Smidt, M.P., and Burbach, J.P. (2006). Spatial and temporal expression of FoxO transcription factors in the developing and adult murine brain. Gene Expr Patterns 6, 134-140.

Hoerder-Suabedissen, A., Wang, W.Z., Lee, S., Davies, K.E., Goffinet, A.M., Rakic, S., Parnavelas, J., Reim, K., Nicolic, M., Paulsen, O., et al. (2008). Novel Markers Reveal Subpopulations of Subplate Neurons in the Murine Cerebral Cortex. Cereb Cortex.

Howell, B.W., Hawkes, R., Soriano, P., and Cooper, J.A. (1997). Neuronal position in the developing brain is regulated by mouse disabled-1. Nature 389, 733-737.

Huang, E.J., and Reichardt, L.F. (2003). Trk receptors: roles in neuronal signal transduction. Annu Rev Biochem 72, 609-642. 
Imura, T., Kornblum, H.I., and Sofroniew, M.V. (2003). The predominant neural stem cell isolated from postnatal and adult forebrain but not early embryonic forebrain expresses GFAP. J Neurosci 23, 2824-2832.

Inglis-Broadgate, S.L., Thomson, R.E., Pellicano, F., Tartaglia, M.A., Pontikis, C.C., Cooper, J.D., and Iwata, T. (2005). FGFR3 regulates brain size by controlling progenitor cell proliferation and apoptosis during embryonic development. Dev Biol 279, 73-85.

Jakobsson, A., Westerberg, R., and Jacobsson, A. (2006). Fatty acid elongases in mammals: their regulation and roles in metabolism. Prog Lipid Res 45, 237-249.

Jani, K., and Schock, F. (2007). Zasp is required for the assembly of functional integrin adhesion sites. J Cell Biol 179, 1583-1597.

Kamei, Y., Inagaki, N., Nishizawa, M., Tsutsumi, O., Taketani, Y., and Inagaki, M. (1998). Visualization of mitotic radial glial lineage cells in the developing rat brain by Cdc2 kinasephosphorylated vimentin. Glia 23, 191-199.

Kaplan, M.H. (2005). STAT4: a critical regulator of inflammation in vivo. Immunol Res 31, 231 242.

Kidd, T., Russell, C., Goodman, C.S., and Tear, G. (1998). Dosage-sensitive and complementary functions of roundabout and commissureless control axon crossing of the CNS midline. Neuron 20, 25-33.

Kim, S.Y., Chung, H.S., Sun, W., and Kim, H. (2007). Spatiotemporal expression pattern of nonclustered protocadherin family members in the developing rat brain. Neuroscience 147, 996-1021.

Ko, J., Humbert, S., Bronson, R.T., Takahashi, S., Kulkarni, A.B., Li, E., and Tsai, L.H. (2001). p35 and p39 are essential for cyclin-dependent kinase 5 function during neurodevelopment. J Neurosci 21, 6758-6771.

Koblar, S.A., Turnley, A.M., Classon, B.J., Reid, K.L., Ware, C.B., Cheema, S.S., Murphy, M., and Bartlett, P.F. (1998). Neural precursor differentiation into astrocytes requires signaling through the leukemia inhibitory factor receptor. Proc Natl Acad Sci U S A 95, 3178-3181.

Koester, S.E., and O'Leary, D.D. (1994). Axons of early generated neurons in cingulate cortex pioneer the corpus callosum. J Neurosci 14, 6608-6620.

Kops, G.J., Medema, R.H., Glassford, J., Essers, M.A., Dijkers, P.F., Coffer, P.J., Lam, E.W., and Burgering, B.M. (2002). Control of cell cycle exit and entry by protein kinase B-regulated forkhead transcription factors. Mol Cell Biol 22, 2025-2036.

Kowalczyk, T., Pontious, A., Englund, C., Daza, R.A., Bedogni, F., Hodge, R., Attardo, A., Bell, C., Huttner, W.B., and Hevner, R.F. (2009). Intermediate Neuronal Progenitors (Basal Progenitors) Produce Pyramidal-Projection Neurons for All Layers of Cerebral Cortex. Cereb Cortex.

Kriegstein, A.R., and Gotz, M. (2003). Radial glia diversity: a matter of cell fate. Glia 43, 37-43.

Kriegstein, A.R., and Noctor, S.C. (2004). Patterns of neuronal migration in the embryonic cortex. Trends Neurosci 27, 392-399. 
Kwan, K.Y., Lam, M.M., Krsnik, Z., Kawasawa, Y.I., Lefebvre, V., and Sestan, N. (2008). SOX5 postmitotically regulates migration, postmigratory differentiation, and projections of subplate and deep-layer neocortical neurons. Proc Natl Acad Sci U S A 105, 16021-16026.

Kwon, Y.T., and Tsai, L.H. (1998). A novel disruption of cortical development in p35(-/-) mice distinct from reeler. J Comp Neurol 395, 510-522.

Lai, T., Jabaudon, D., Molyneaux, B.J., Azim, E., Arlotta, P., Menezes, J.R., and Macklis, J.D. (2008). SOX5 controls the sequential generation of distinct corticofugal neuron subtypes. Neuron 57, 232-247.

Laywell, E.D., Rakic, P., Kukekov, V.G., Holland, E.C., and Steindler, D.A. (2000). Identification of a multipotent astrocytic stem cell in the immature and adult mouse brain. Proc Natl Acad Sci U S A 97, 13883-13888.

Leone, D.P., Srinivasan, K., Chen, B., Alcamo, E., and McConnell, S.K. (2008). The determination of projection neuron identity in the developing cerebral cortex. Curr Opin Neurobiol 18, 28-35.

Levison, S.W., and Goldman, J.E. (1997). Multipotential and lineage restricted precursors coexist in the mammalian perinatal subventricular zone. J Neurosci Res 48, 83-94.

Li, W., Cogswell, C.A., and LoTurco, J.J. (1998). Neuronal differentiation of precursors in the neocortical ventricular zone is triggered by BMP. J Neurosci 18, 8853-8862.

Liapi, A., Pritchett, J., Jones, O., Fujii, N., Parnavelas, J.G., and Nadarajah, B. (2008). Stromalderived factor 1 signalling regulates radial and tangential migration in the developing cerebral cortex. Dev Neurosci 30, 117-131.

Lim, R., Liu, Y.X., and Zaheer, A. (1990). Cell-surface expression of glia maturation factor beta in astrocytes. FASEB J 4, 3360-3363.

Lim, R., Miller, J.F., and Zaheer, A. (1989). Purification and characterization of glia maturation factor beta: a growth regulator for neurons and glia. Proc Natl Acad Sci U S A 86, 3901-3905.

Livy, D.J., Schalomon, P.M., Roy, M., Zacharias, M.C., Pimenta, J., Lent, R., and Wahlsten, D. (1997). Increased axon number in the anterior commissure of mice lacking a corpus callosum. Exp Neurol 146, 491-501.

Lopez-Bendito, G., Flames, N., Ma, L., Fouquet, C., Di Meglio, T., Chedotal, A., Tessier-Lavigne, M., and Marin, O. (2007). Robo1 and Robo2 cooperate to control the guidance of major axonal tracts in the mammalian forebrain. J Neurosci 27, 3395-3407.

Louvi, A., and Artavanis-Tsakonas, S. (2006). Notch signalling in vertebrate neural development. Nat Rev Neurosci 7, 93-102.

Lukaszewicz, A., Savatier, P., Cortay, V., Kennedy, H., and Dehay, C. (2002). Contrasting effects of basic fibroblast growth factor and neurotrophin 3 on cell cycle kinetics of mouse cortical stem cells. J Neurosci 22, 6610-6622.

Magdaleno, S., Keshvara, L., and Curran, T. (2002). Rescue of ataxia and preplate splitting by ectopic expression of Reelin in reeler mice. Neuron 33, 573-586. 
Malatesta, P., Hack, M.A., Hartfuss, E., Kettenmann, H., Klinkert, W., Kirchhoff, F., and Gotz, M. (2003). Neuronal or glial progeny: regional differences in radial glia fate. Neuron 37, 751-764.

Mallamaci, A., Muzio, L., Chan, C.H., Parnavelas, J., and Boncinelli, E. (2000). Area identity shifts in the early cerebral cortex of Emx2-/- mutant mice. Nat Neurosci 3, 679-686.

Marshall, C.A., Novitch, B.G., and Goldman, J.E. (2005). Olig2 directs astrocyte and oligodendrocyte formation in postnatal subventricular zone cells. J Neurosci 25, 7289-7298.

Maruhashi, M., Van De Putte, T., Huylebroeck, D., Kondoh, H., and Higashi, Y. (2005). Involvement of SIP1 in positioning of somite boundaries in the mouse embryo. Dev Dyn 234, 332 338.

Matthews, J.M., and Visvader, J.E. (2003). LIM-domain-binding protein 1: a multifunctional cofactor that interacts with diverse proteins. EMBO Rep 4, 1132-1137.

McCarthy, M., Turnbull, D.H., Walsh, C.A., and Fishell, G. (2001). Telencephalic neural progenitors appear to be restricted to regional and glial fates before the onset of neurogenesis. $\mathrm{J}$ Neurosci 21,6772-6781.

Mehler, M.F., Mabie, P.C., Zhang, D., and Kessler, J.A. (1997). Bone morphogenetic proteins in the nervous system. Trends Neurosci 20, 309-317.

Menard, C., Hein, P., Paquin, A., Savelson, A., Yang, X.M., Lederfein, D., Barnabe-Heider, F., Mir, A.A., Sterneck, E., Peterson, A.C., et al. (2002). An essential role for a MEK-C/EBP pathway during growth factor-regulated cortical neurogenesis. Neuron 36, 597-610.

Meylan, E., Martinon, F., Thome, M., Gschwendt, M., and Tschopp, J. (2002). RIP4 (DIK/PKK), a novel member of the RIP kinase family, activates NF-kappa B and is processed during apoptosis. EMBO Rep 3, 1201-1208.

Miller, F.D., and Gauthier, A.S. (2007). Timing is everything: making neurons versus glia in the developing cortex. Neuron 54, 357-369.

Miquelajauregui, A., Van de Putte, T., Polyakov, A., Nityanandam, A., Boppana, S., Seuntjens, E., Karabinos, A., Higashi, Y., Huylebroeck, D., and Tarabykin, V. (2007). Smad-interacting protein-1 (Zfhxlb) acts upstream of Wnt signaling in the mouse hippocampus and controls its formation. Proc Natl Acad Sci U S A 104, 12919-12924.

Misson, J.P., Edwards, M.A., Yamamoto, M., and Caviness, V.S., Jr. (1988). Identification of radial glial cells within the developing murine central nervous system: studies based upon a new immunohistochemical marker. Brain Res Dev Brain Res 44, 95-108.

Miura, E., Iijima, T., Yuzaki, M., and Watanabe, M. (2006). Distinct expression of Cbln family mRNAs in developing and adult mouse brains. Eur J Neurosci 24, 750-760.

Miyata, T., Kawaguchi, A., Saito, K., Kawano, M., Muto, T., and Ogawa, M. (2004). Asymmetric production of surface-dividing and non-surface-dividing cortical progenitor cells. Development $131,3133-3145$. 
Mizuno, N., Kokubu, H., Sato, M., Nishimura, A., Yamauchi, J., Kurose, H., and Itoh, H. (2005). G protein-coupled receptor signaling through Gq and JNK negatively regulates neural progenitor cell migration. Proc Natl Acad Sci U S A 102, 12365-12370.

Molne, M., Studer, L., Tabar, V., Ting, Y.T., Eiden, M.V., and McKay, R.D. (2000). Early cortical precursors do not undergo LIF-mediated astrocytic differentiation. J Neurosci Res 59, 301-311.

Molyneaux, B.J., Arlotta, P., Hirata, T., Hibi, M., and Macklis, J.D. (2005). Fezl is required for the birth and specification of corticospinal motor neurons. Neuron 47, 817-831.

Monuki, E.S., and Walsh, C.A. (2001). Mechanisms of cerebral cortical patterning in mice and humans. Nat Neurosci 4 Suppl, 1199-1206.

Morrison, S.J., Perez, S.E., Qiao, Z., Verdi, J.M., Hicks, C., Weinmaster, G., and Anderson, D.J. (2000). Transient Notch activation initiates an irreversible switch from neurogenesis to gliogenesis by neural crest stem cells. Cell 101, 499-510.

Morrow, T., Song, M.R., and Ghosh, A. (2001). Sequential specification of neurons and glia by developmentally regulated extracellular factors. Development 128, 3585-3594.

Mukhopadhyay, M., Teufel, A., Yamashita, T., Agulnick, A.D., Chen, L., Downs, K.M., Schindler, A., Grinberg, A., Huang, S.P., Dorward, D., et al. (2003). Functional ablation of the mouse Ldb1 gene results in severe patterning defects during gastrulation. Development 130, 495-505.

Muzio, L., DiBenedetto, B., Stoykova, A., Boncinelli, E., Gruss, P., and Mallamaci, A. (2002). Conversion of cerebral cortex into basal ganglia in Emx2(-/-) Pax6(Sey/Sey) double-mutant mice. Nat Neurosci 5, 737-745.

Muzio, L., and Mallamaci, A. (2003). Emx1, emx2 and pax6 in specification, regionalization and arealization of the cerebral cortex. Cereb Cortex 13, 641-647.

Nadarajah, B. (2003). Radial glia and somal translocation of radial neurons in the developing cerebral cortex. Glia 43, 33-36.

Nadarajah, B., Alifragis, P., Wong, R.O., and Parnavelas, J.G. (2003). Neuronal migration in the developing cerebral cortex: observations based on real-time imaging. Cereb Cortex 13, 607-611.

Nadarajah, B., Brunstrom, J.E., Grutzendler, J., Wong, R.O., and Pearlman, A.L. (2001). Two modes of radial migration in early development of the cerebral cortex. Nat Neurosci 4, 143-150.

Nakamura, S., Todo, T., Motoi, Y., Haga, S., Aizawa, T., Ueki, A., and Ikeda, K. (1999). Glial expression of fibroblast growth factor-9 in rat central nervous system. Glia 28, 53-65.

Nakashima, K., Takizawa, T., Ochiai, W., Yanagisawa, M., Hisatsune, T., Nakafuku, M., Miyazono, K., Kishimoto, T., Kageyama, R., and Taga, T. (2001). BMP2-mediated alteration in the developmental pathway of fetal mouse brain cells from neurogenesis to astrocytogenesis. Proc Natl Acad Sci U S A 98, 5868-5873.

Nakashima, K., Wiese, S., Yanagisawa, M., Arakawa, H., Kimura, N., Hisatsune, T., Yoshida, K., Kishimoto, T., Sendtner, M., and Taga, T. (1999a). Developmental requirement of gp130 signaling in neuronal survival and astrocyte differentiation. J Neurosci 19, 5429-5434. 
Nakashima, K., Yanagisawa, M., Arakawa, H., Kimura, N., Hisatsune, T., Kawabata, M., Miyazono, K., and Taga, T. (1999b). Synergistic signaling in fetal brain by STAT3-Smad1 complex bridged by p300. Science 284, 479-482.

Nieto, M., Schuurmans, C., Britz, O., and Guillemot, F. (2001). Neural bHLH genes control the neuronal versus glial fate decision in cortical progenitors. Neuron 29, 401-413.

Nitta, K.R., Tanegashima, K., Takahashi, S., and Asashima, M. (2004). XSIP1 is essential for early neural gene expression and neural differentiation by suppression of BMP signaling. Dev Biol 275, $258-267$.

Noctor, S.C., Flint, A.C., Weissman, T.A., Dammerman, R.S., and Kriegstein, A.R. (2001). Neurons derived from radial glial cells establish radial units in neocortex. Nature 409, 714-720.

Noctor, S.C., Martinez-Cerdeno, V., Ivic, L., and Kriegstein, A.R. (2004). Cortical neurons arise in symmetric and asymmetric division zones and migrate through specific phases. Nat Neurosci 7, 136-144.

Noctor, S.C., Palmer, S.L., Hasling, T., and Juliano, S.L. (1999). Interference with the development of early generated neocortex results in disruption of radial glia and abnormal formation of neocortical layers. Cereb Cortex 9, 121-136.

Ohkubo, Y., Uchida, A.O., Shin, D., Partanen, J., and Vaccarino, F.M. (2004). Fibroblast growth factor receptor 1 is required for the proliferation of hippocampal progenitor cells and for hippocampal growth in mouse. J Neurosci 24, 6057-6069.

Ohshima, T., Ogawa, M., Veeranna, Hirasawa, M., Longenecker, G., Ishiguro, K., Pant, H.C., Brady, R.O., Kulkarni, A.B., and Mikoshiba, K. (2001). Synergistic contributions of cyclindependant kinase 5/p35 and Reelin/Dab1 to the positioning of cortical neurons in the developing mouse brain. Proc Natl Acad Sci U S A 98, 2764-2769.

Paquin, A., Barnabe-Heider, F., Kageyama, R., and Miller, F.D. (2005). CCAAT/enhancer-binding protein phosphorylation biases cortical precursors to generate neurons rather than astrocytes in vivo. J Neurosci 25, 10747-10758.

Parnavelas, J.G. (1999). Glial cell lineages in the rat cerebral cortex. Exp Neurol 156, 418-429.

Pearson, B.J., and Doe, C.Q. (2004). Specification of temporal identity in the developing nervous system. Annu Rev Cell Dev Biol 20, 619-647.

Polleux, F., Dehay, C., Goffinet, A., and Kennedy, H. (2001). Pre- and post-mitotic events contribute to the progressive acquisition of area-specific connectional fate in the neocortex. Cereb Cortex 11, 1027-1039.

Polleux, F., Dehay, C., and Kennedy, H. (1998). Neurogenesis and commitment of corticospinal neurons in reeler. J Neurosci 18, 9910-9923.

Pringle, N.P., Yu, W.P., Howell, M., Colvin, J.S., Ornitz, D.M., and Richardson, W.D. (2003). Fgfr3 expression by astrocytes and their precursors: evidence that astrocytes and oligodendrocytes originate in distinct neuroepithelial domains. Development 130, 93-102. 
Qian, X., Shen, Q., Goderie, S.K., He, W., Capela, A., Davis, A.A., and Temple, S. (2000). Timing of CNS cell generation: a programmed sequence of neuron and glial cell production from isolated murine cortical stem cells. Neuron 28, 69-80.

Rakic, P. (2007). The radial edifice of cortical architecture: from neuronal silhouettes to genetic engineering. Brain Res Rev 55, 204-219.

Rash, B.G., and Richards, L.J. (2001). A role for cingulate pioneering axons in the development of the corpus callosum. J Comp Neurol 434, 147-157.

Reid, C.B., Liang, I., and Walsh, C. (1995). Systematic widespread clonal organization in cerebral cortex. Neuron 15, 299-310.

Remacle, J.E., Kraft, H., Lerchner, W., Wuytens, G., Collart, C., Verschueren, K., Smith, J.C., and Huylebroeck, D. (1999). New mode of DNA binding of multi-zinc finger transcription factors: deltaEF1 family members bind with two hands to two target sites. EMBO J 18, 5073-5084.

Rousselot, P., Heintz, N., and Nottebohm, F. (1997). Expression of brain lipid binding protein in the brain of the adult canary and its implications for adult neurogenesis. J Comp Neurol 385, 415426.

Sardi, S.P., Murtie, J., Koirala, S., Patten, B.A., and Corfas, G. (2006). Presenilin-dependent ErbB4 nuclear signaling regulates the timing of astrogenesis in the developing brain. Cell 127, 185-197.

Sauer, M.E., and Walker, B.E. (1959). Radioautographic study of interkinetic nuclear migration in the neural tube. Proc Soc Exp Biol Med 101, 557-560.

Schmid, R.S., McGrath, B., Berechid, B.E., Boyles, B., Marchionni, M., Sestan, N., and Anton, E.S. (2003). Neuregulin 1-erbB2 signaling is required for the establishment of radial glia and their transformation into astrocytes in cerebral cortex. Proc Natl Acad Sci U S A 100, 4251-4256.

Schmid, R.S., Shelton, S., Stanco, A., Yokota, Y., Kreidberg, J.A., and Anton, E.S. (2004). alpha3beta1 integrin modulates neuronal migration and placement during early stages of cerebral cortical development. Development 131, 6023-6031.

Schmidt, M., Fernandez de Mattos, S., van der Horst, A., Klompmaker, R., Kops, G.J., Lam, E.W., Burgering, B.M., and Medema, R.H. (2002). Cell cycle inhibition by FoxO forkhead transcription factors involves downregulation of cyclin D. Mol Cell Biol 22, 7842-7852.

Scholzen, T., and Gerdes, J. (2000). The Ki-67 protein: from the known and the unknown. J Cell Physiol 182, 311-322.

Schuurmans, C., Armant, O., Nieto, M., Stenman, J.M., Britz, O., Klenin, N., Brown, C., Langevin, L.M., Seibt, J., Tang, H., et al. (2004). Sequential phases of cortical specification involve Neurogenin-dependent and -independent pathways. EMBO J 23, 2892-2902.

Seoane, J., Le, H.V., Shen, L., Anderson, S.A., and Massague, J. (2004). Integration of Smad and forkhead pathways in the control of neuroepithelial and glioblastoma cell proliferation. Cell 117, 211-223. 
Sheldon, M., Rice, D.S., D'Arcangelo, G., Yoneshima, H., Nakajima, K., Mikoshiba, K., Howell, B.W., Cooper, J.A., Goldowitz, D., and Curran, T. (1997). Scrambler and yotari disrupt the disabled gene and produce a reeler-like phenotype in mice. Nature 389, 730-733.

Shen, Q., Wang, Y., Dimos, J.T., Fasano, C.A., Phoenix, T.N., Lemischka, I.R., Ivanova, N.B., Stifani, S., Morrisey, E.E., and Temple, S. (2006). The timing of cortical neurogenesis is encoded within lineages of individual progenitor cells. Nat Neurosci 9, 743-751.

Sheppard, A.M., and Pearlman, A.L. (1997). Abnormal reorganization of preplate neurons and their associated extracellular matrix: an early manifestation of altered neocortical development in the reeler mutant mouse. J Comp Neurol 378, 173-179.

Smith, K.M., Ohkubo, Y., Maragnoli, M.E., Rasin, M.R., Schwartz, M.L., Sestan, N., and Vaccarino, F.M. (2006). Midline radial glia translocation and corpus callosum formation require FGF signaling. Nat Neurosci 9, 787-797.

Song, M.R., and Ghosh, A. (2004). FGF2-induced chromatin remodeling regulates CNTF-mediated gene expression and astrocyte differentiation. Nat Neurosci 7, 229-235.

Sugitani, Y., Nakai, S., Minowa, O., Nishi, M., Jishage, K., Kawano, H., Mori, K., Ogawa, M., and Noda, T. (2002). Brn-1 and Brn-2 share crucial roles in the production and positioning of mouse neocortical neurons. Genes Dev 16, 1760-1765.

Sun, Y., Goderie, S.K., and Temple, S. (2005). Asymmetric distribution of EGFR receptor during mitosis generates diverse CNS progenitor cells. Neuron 45, 873-886.

Sun, Y., Nadal-Vicens, M., Misono, S., Lin, M.Z., Zubiaga, A., Hua, X., Fan, G., and Greenberg, M.E. (2001). Neurogenin promotes neurogenesis and inhibits glial differentiation by independent mechanisms. Cell 104, 365-376.

Takahashi, T., Nowakowski, R.S., and Caviness, V.S., Jr. (1995). The cell cycle of the pseudostratified ventricular epithelium of the embryonic murine cerebral wall. J Neurosci 15, 60466057.

Takiguchi-Hayashi, K., Sekiguchi, M., Ashigaki, S., Takamatsu, M., Hasegawa, H., SuzukiMigishima, R., Yokoyama, M., Nakanishi, S., and Tanabe, Y. (2004). Generation of reelin-positive marginal zone cells from the caudomedial wall of telencephalic vesicles. J Neurosci 24, 2286-2295. Tamai, H., Shinohara, H., Miyata, T., Saito, K., Nishizawa, Y., Nomura, T., and Osumi, N. (2007). Pax6 transcription factor is required for the interkinetic nuclear movement of neuroepithelial cells. Genes Cells 12, 983-996.

Tanigaki, K., Nogaki, F., Takahashi, J., Tashiro, K., Kurooka, H., and Honjo, T. (2001). Notch1 and Notch3 instructively restrict bFGF-responsive multipotent neural progenitor cells to an astroglial fate. Neuron 29, 45-55.

Thomson, R.E., Pellicano, F., and Iwata, T. (2007). Fibroblast growth factor receptor 3 kinase domain mutation increases cortical progenitor proliferation via mitogen-activated protein kinase activation. J Neurochem 100, 1565-1578. 
Tissir, F., and Goffinet, A.M. (2003). Reelin and brain development. Nat Rev Neurosci 4, 496-505. Todo, T., Kondo, T., Nakamura, S., Kirino, T., Kurokawa, T., and Ikeda, K. (1998). Neuronal localization of fibroblast growth factor-9 immunoreactivity in human and rat brain. Brain Res 783, 179-187.

Trommsdorff, M., Gotthardt, M., Hiesberger, T., Shelton, J., Stockinger, W., Nimpf, J., Hammer, R.E., Richardson, J.A., and Herz, J. (1999). Reeler/Disabled-like disruption of neuronal migration in knockout mice lacking the VLDL receptor and ApoE receptor 2. Cell 97, 689-701.

Vaccarino, F.M., Schwartz, M.L., Raballo, R., Nilsen, J., Rhee, J., Zhou, M., Doetschman, T., Coffin, J.D., Wyland, J.J., and Hung, Y.T. (1999). Changes in cerebral cortex size are governed by fibroblast growth factor during embryogenesis. Nat Neurosci 2, 246-253.

Van de Putte, T., Maruhashi, M., Francis, A., Nelles, L., Kondoh, H., Huylebroeck, D., and Higashi, Y. (2003). Mice lacking ZFHX1B, the gene that codes for Smad-interacting protein-1, reveal a role for multiple neural crest cell defects in the etiology of Hirschsprung disease-mental retardation syndrome. Am J Hum Genet 72, 465-470.

van Grunsven, L.A., Michiels, C., Van de Putte, T., Nelles, L., Wuytens, G., Verschueren, K., and Huylebroeck, D. (2003). Interaction between Smad-interacting protein-1 and the corepressor Cterminal binding protein is dispensable for transcriptional repression of E-cadherin. J Biol Chem $278,26135-26145$.

van Grunsven, L.A., Taelman, V., Michiels, C., Opdecamp, K., Huylebroeck, D., and Bellefroid, E.J. (2006). deltaEF1 and SIP1 are differentially expressed and have overlapping activities during Xenopus embryogenesis. Dev Dyn 235, 1491-1500.

Vandewalle, C., Comijn, J., De Craene, B., Vermassen, P., Bruyneel, E., Andersen, H., Tulchinsky, E., Van Roy, F., and Berx, G. (2005). SIP1/ZEB2 induces EMT by repressing genes of different epithelial cell-cell junctions. Nucleic Acids Res 33, 6566-6578.

Vanhalst, K., Kools, P., Staes, K., van Roy, F., and Redies, C. (2005). delta-Protocadherins: a gene family expressed differentially in the mouse brain. Cell Mol Life Sci 62, 1247-1259.

Verschueren, K., Remacle, J.E., Collart, C., Kraft, H., Baker, B.S., Tylzanowski, P., Nelles, L., Wuytens, G., Su, M.T., Bodmer, R., et al. (1999). SIP1, a novel zinc finger/homeodomain repressor, interacts with Smad proteins and binds to 5'-CACCT sequences in candidate target genes. J Biol Chem 274, 20489-20498.

Viti, J., Feathers, A., Phillips, J., and Lillien, L. (2003). Epidermal growth factor receptors control competence to interpret leukemia inhibitory factor as an astrocyte inducer in developing cortex. $\mathrm{J}$ Neurosci 23, 3385-3393.

Wakamatsu, N., Yamada, Y., Yamada, K., Ono, T., Nomura, N., Taniguchi, H., Kitoh, H., Mutoh, N., Yamanaka, T., Mushiake, K., et al. (2001). Mutations in SIP1, encoding Smad interacting protein-1, cause a form of Hirschsprung disease. Nat Genet 27, 369-370. 
Walsh, C., and Cepko, C.L. (1993). Clonal dispersion in proliferative layers of developing cerebral cortex. Nature 362, 632-635.

Wilkie, A.L., Jordan, S.A., Sharpe, J.A., Price, D.J., and Jackson, I.J. (2004). Widespread tangential dispersion and extensive cell death during early neurogenesis in the mouse neocortex. Dev Biol 267, 109-118.

Wilson, S.W., and Rubenstein, J.L. (2000). Induction and dorsoventral patterning of the telencephalon. Neuron 28, 641-651.

Xu, Q., de la Cruz, E., and Anderson, S.A. (2003). Cortical interneuron fate determination: diverse sources for distinct subtypes? Cereb Cortex 13, 670-676.

Yanagisawa, M., Takizawa, T., Ochiai, W., Uemura, A., Nakashima, K., and Taga, T. (2001). Fate alteration of neuroepithelial cells from neurogenesis to astrocytogenesis by bone morphogenetic proteins. Neurosci Res 41, 391-396.

Yoon, K., and Gaiano, N. (2005). Notch signaling in the mammalian central nervous system: insights from mouse mutants. Nat Neurosci 8, 709-715.

Yoon, K., Nery, S., Rutlin, M.L., Radtke, F., Fishell, G., and Gaiano, N. (2004). Fibroblast growth factor receptor signaling promotes radial glial identity and interacts with Notch1 signaling in telencephalic progenitors. J Neurosci 24, 9497-9506.

Yoshida, M., Suda, Y., Matsuo, I., Miyamoto, N., Takeda, N., Kuratani, S., and Aizawa, S. (1997). Emx1 and Emx2 functions in development of dorsal telencephalon. Development 124, 101-111.

Yoshimoto, A., Saigou, Y., Higashi, Y., and Kondoh, H. (2005). Regulation of ocular lens development by Smad-interacting protein 1 involving Foxe3 activation. Development 132, 44374448.

Yun, K., Mantani, A., Garel, S., Rubenstein, J., and Israel, M.A. (2004). Id4 regulates neural progenitor proliferation and differentiation in vivo. Development 131, 5441-5448.

Zaheer, A., Yorek, M.A., and Lim, R. (2001). Effects of glia maturation factor overexpression in primary astrocytes on MAP kinase activation, transcription factor activation, and neurotrophin secretion. Neurochem Res 26, 1293-1299.

Zhao, S., Hung, F.C., Colvin, J.S., White, A., Dai, W., Lovicu, F.J., Ornitz, D.M., and Overbeek, P.A. (2001). Patterning the optic neuroepithelium by FGF signaling and Ras activation. Development 128, 5051-5060.

Zweier, C., Albrecht, B., Mitulla, B., Behrens, R., Beese, M., Gillessen-Kaesbach, G., Rott, H.D., and Rauch, A. (2002). "Mowat-Wilson" syndrome with and without Hirschsprung disease is a distinct, recognizable multiple congenital anomalies-mental retardation syndrome caused by mutations in the zinc finger homeo box 1B gene. Am J Med Genet 108, 177-181. 


\section{ACKNOWLEDGEMENT}

I would like to hereby express my heartfelt gratitude to Dr. Victor Tarabykin for his excellent supervision, encouragement and support throughout the course of my PhD; Prof. Walter Stühmer, Prof. Klaus- Armin Nave and Prof. Kerstin Krieglstein for very helpful suggestions and constructive criticism of my work as my thesis committee members; Dr. Eve Seuntjens and Prof. Danny Huylebroeck, our long-standing collaborators at the University of Leuven; NEUREST, for the financial support without which this project would not have been possible; Dr. Miso Mitkovski, for teaching me everything I know today about Microscopy and Image analysis, for his patience and magnanimity; Marten Kuscher, my former Diploma student, for his sincerity and dedication in assisting me with various experiments, for being a wonderful person and a very dear friend; Mrinalini Hoon, for her extremely useful comments on my thesis, for being my pillar of support time and again, and for the amazing friendship that we share; my former and current colleagues in the lab: Amaya Miquelajauregui, for handing me this project on a platter and for guiding me through the initial phase of my PhD, Malte Puhan, for being one of the most selfless people I have ever met, Manuela Schwark, for inspiring me with her perfectionism, Sridhar Boppana, for being an elder brother to me, Elena Kvashnina, for being one of the most kind and generous people I have known, and Vivi Sgourdou, for all the fun moments we have shared. A very special thanks also to all members of Prof. Stühmer's department, for their warmth and friendliness, and for leaving me with very fond memories.

I am deeply indebted to my close friends, Ivana Gadjanski, Veronique Planchamp, Camino de Juan, Mrinalini Hoon and Sailu Mandalapu, for always being there for me, and for making my stay in Goettingen absolutely unforgettable.

My years in Goettingen have been enormously enriching, to say the least, and that would not have been possible without the International Neuroscience Program; it has been a pleasure to be a part of this venture.

Finally, a very big thank you to my parents (I wouldn't be anywhere without them) and the one person I love more than anyone else in the world, my brother Maruthi. Thank you for everything. 


\title{
CURRICULUM VITAE
}

\author{
ANJANA NITYANANDAM \\ Cortical Development Group \\ Max Planck Institute for Experimental Medicine \\ Hermann Rein strasse 3, \\ 37075 Goettingen \\ Germany \\ Phone - 004917624331191 (Mobile) \\ 00495513899372 (Office) \\ 00495513072881 (Home) \\ Email - nityanandam@em.mpg.de
}

Date and Place of Birth

\section{Education}

B.Tech (Biotechnology)

Aug 1999 to Jul 2003

Guru Gobind Singh Indraprastha University

Delhi, India

MSc (Integrated MSc/PhD) (Neuroscience) Sep 2004 Aug 2005

International Max Planck Research School

University of Goettingen

Germany

\section{PhD (Neuroscience)}

Oct 2005 to present

Max Planck Institute for Experimental medicine

University of Goettingen

Germany

\section{Research Experience}

1. Identification of neural crest cells in developing chick embryo; basic techniques in chick embryology including histology and in vitro culturing;

JNCASR summer research fellowship, Pune, India; June- July 2002.

2. Isolation, purification and characterisation of ubiquitin from goat erythrocytes; B.Tech, GGSIPU, Delhi, India; Jan-Jul 2003.

3. Morphological effects of ubiquitin on brain development in chick embryo; GGSIPU, Delhi, India; Aug 2003- Aug 2004. 
Lab Rotation Projects

4. Study of KATP channel currents expressed in HEK cells and cytoskeletal rearrangements as a consequence of the expression; Lab Rotation 1, MSc/PhD program in Neuroscience, Jan- Feb 2005.

5. Studies on the spatial regulation of FRET efficiency between mCerulean and mVenus in RMCD cells; Lab Rotation 2, MSc/PhD program in Neuroscience, Feb- Mar 2005.

6. Effect of ectopic overexpression of Satb2 transcription factor on neocortical development; Lab Rotation 3, MSc/PhD program in Neuroscience, Apr- May 2005.

7. Specification of serotonergic neurons in ventral hindbrain primary culture and neural stem cell line; Lab Rotation 4, MSc/PhD program in Neuroscience, May-Jul 2005.

$\mathrm{PhD}$ project

8. Investigation of Sip1 gene interactions in the development of the mammalian telencephalon; $\mathrm{PhD}$, Max Planck Institute for Experimental Medicine, Goettingen, Germany; Oct 2005 to present.

\section{Scientific presentations}

1. Presented a poster titled 'Ubiquitin as a novel tool to study morphological changes during early neural development with chick embryos as a model system' at Neurizons (organised by the international Max Planck Research School in Max Planck Institute for Experimental Medicine, Goettingen) in Nov 2004, Goettingen.

2. Presented a poster titled 'Sip1 regulates proliferation, differentiation and migration in the embryonic mouse neocortex' at the 7th Goettingen Meeting of the German Neuroscience Society, Mar 29 - Apr 1 2007, Goettingen.

3. Presented a poster titled 'Sip1 regulates proliferation, differentiation and migration in the embryonic mouse neocortex' at the 2007 Brain Development Symposium in London (10th May 2007)

4. Presented a poster titled 'Sip1 regulates proliferation, differentiation and migration in the embryonic mouse neocortex' at the 7th IBRO World Congress of Neuroscience in Melbourne (12th - 17th Jul 2007)

5. Presented a poster titled 'Sip1 regulates proliferation, differentiation and migration in the embryonic mouse neocortex' at Neurizons 2007 in Goettingen (31st May - 2nd Jun 2007)

6. Presented a poster titled 'Sip1 regulates proliferation, differentiation and migration in the embryonic mouse neocortex' at Horizons 2007 in Goettingen (12- 15 Sep 2007)

7. Presented a talk titled 'Sip1 regulates proliferation, differentiation and migration in the embryonic mouse neocortex' at Neuroscience 2007, Annual meeting of the Society for 
Neuroscience in San Diego, USA (3- 7 Nov 2007)

8. Presented a poster titled 'Sip1 regulates astrogliogenesis, neuronal migration and postnatal proliferation during neocortical development' at Cortical Development Meeting 2008 in Chania, Greece (22- 25 May 2008)

\section{Publications}

1. Amaya Miquelajauregui, Tom Van de Putte, Alexander Polyakov, Anjana Nityanandam, Eve Seuntjens, Yujiro Higashi, Danny Huylebroeck and Victor Tarabykin, Smad-interacting protein-1 (Sip1/Zfhx1b) acts upstream of Wnt signalling in the control of hippocampus formation in the mouse, PNAS, Jul 2007.

2. Eve Seuntjens*, Anjana Nityanandam*, Amaya Miquelajauregui, Joke Debruyn, A Stryjewska, Sandra Goebbels, Klaus- Armin Nave, Danny Huylebroeck and Victor Tarabykin, Sip1 (Zfhxlb) regulates sequential fate decisions through feedback signalling from postmitotic neurons to progenitor cells, (requested for resubmission to Nature Neuroscience after review).

(* contributed equally to the paper)

\section{Awards and Honours}

1. Gold medallist in B.Tech (1999-2003)

2. Recipient of JNCASR Summer Research Fellowship, June - July 2002

3. Recipient of International Max Planck Research School Stipend; Sep 2004Aug 2005

4. Recipient of the NEUREST (Marie Curie Actions/ EU Programme) fellowship; Oct 2005 Sep 2008.

\section{Communication Skills}

Presented my PhD work at various scientific Symposia, departmental meetings and retreats.

Attended several courses on scientific presentation and publishing, and Science and medical writing. 


\section{PUBLICATION LIST}

1. Amaya Miquelajauregui, Tom Van de Putte, Alexander Polyakov, Anjana Nityanandam, Eve Seuntjens, Yujiro Higashi, Danny Huylebroeck and Victor Tarabykin, Smad-interacting protein-1 (Sip1/Zfhx1b) acts upstream of Wnt signalling in the control of hippocampus formation in the mouse, PNAS, Jul 2007.

2. Eve Seuntjens*, Anjana Nityanandam*, Amaya Miquelajauregui, Joke Debruyn, A Stryjewska, Sandra Goebbels, Klaus- Armin Nave, Danny Huylebroeck and Victor Tarabykin, Sip1 (Zfhxlb) regulates sequential fate decisions through feedback signalling from postmitotic neurons to progenitor cells; (requested for resubmission to Nature Neuroscience after review). (* contributed equally to the paper) 